\title{
GAS, STARS, AND STAR FORMATION IN ALFALFA DWARF GALAXIES*
}

\author{
Shan Huang ${ }^{1}$, Martha P. Haynes ${ }^{1}$, Riccardo Giovanelli ${ }^{1}$, Jarle Brinchmann ${ }^{2}$, \\ Sabrina Stierwalt ${ }^{3}$, and Susan G. Neff ${ }^{4}$ \\ ${ }^{1}$ Center for Radiophysics and Space Research, Space Sciences Building, Cornell University, Ithaca, NY 14853, USA; \\ shan@astro.cornell.edu, haynes@astro.cornell.edu, riccardo@astro.cornell.edu \\ 2 Sterrewacht Leiden, Leiden University, NL-2300 RA Leiden, The Netherlands; jarle@strw.leidenuniv.nl \\ ${ }^{3}$ Spitzer Science Center, California Institute of Technology, 1200 E. California Blvd., Pasadena, CA 91125, USA; sabrina@ipac.caltech.edu \\ ${ }^{4}$ NASA GSFC, Code 665, Observational Cosmology Lab, Greenbelt, MD 20771, USA; susan.g.neff@nasa.gov \\ Received 2011 October 7; accepted 2012 March 11; published 2012 April 30
}

\begin{abstract}
We examine the global properties of the stellar and $\mathrm{Hi}$ components of 229 low $\mathrm{Hi}$ mass dwarf galaxies extracted from the ALFALFA survey, including a complete sample of 176 galaxies with $\mathrm{Hi}$ masses $<10^{7.7} M_{\cup}$ and $\mathrm{Hi}$ line widths $<80 \mathrm{~km} \mathrm{~s}^{-1}$. Sloan Digital Sky Survey (SDSS) data are combined with photometric properties derived from Galaxy Evolution Explorer to derive stellar masses $\left(M_{*}\right)$ and star formation rates (SFRs) by fitting their UV-optical spectral energy distributions (SEDs). In optical images, many of the ALFALFA dwarfs are faint and of low surface brightness; only $56 \%$ of those within the SDSS footprint have a counterpart in the SDSS spectroscopic survey. A large fraction of the dwarfs have high specific star formation rates (SSFRs), and estimates of their SFRs and $M_{*}$ obtained by SED fitting are systematically smaller than ones derived via standard formulae assuming a constant SFR. The increased dispersion of the SSFR distribution at $M_{*}>10^{8} M_{0}$ is driven by a set of dwarf galaxies that have low gas fractions and SSFRs; some of these are $\mathrm{dE} / \mathrm{dSphs}$ in the Virgo Cluster. The imposition of an upper Hi mass limit yields the selection of a sample with lower gas fractions for their $M_{*}$ than found for the overall ALFALFA population. Many of the ALFALFA dwarfs, particularly the Virgo members, have H i depletion timescales shorter than a Hubble time. An examination of the dwarf galaxies within the full ALFALFA population in the context of global star formation (SF) laws is consistent with the general assumptions that gas-rich galaxies have lower SF efficiencies than do optically selected populations and that $\mathrm{H}$ i disks are more extended than stellar ones.
\end{abstract}

Key words: galaxies: dwarf - galaxies: evolution - galaxies: fundamental parameters - galaxies: star formation radio lines: galaxies - surveys

Online-only material: color figures, machine-readable and VO tables

\section{INTRODUCTION}

Principal aims of current studies of galaxy formation and evolution include the exploration of the interplay between the gaseous and stellar components of galaxies and the mechanisms that trigger the conversion of gas into stars. During the last decade, wide-area surveys such as the Sloan Digital Sky Survey (SDSS) and the Galaxy Evolution Explorer (GALEX) have enabled statistical studies of star formation (SF) in the local universe. For example, galaxies that are currently forming stars, the so-called blue-cloud galaxies in the color-magnitude diagram (CMD; Baldry et al. 2004), occupy a relatively narrow "star-forming sequence" in a plot of specific star formation rate $\left(\mathrm{SSFR}=\mathrm{SFR} / M_{*}\right)$ versus stellar mass $M_{*}$ (Brinchmann et al. 2004; Salim et al. 2007), with the SSFR declining as the stellar mass increases. Such a trend suggests that the galaxy's stellar mass regulates the overall star formation history (SFH), at least at intermediate masses. This star-forming sequence breaks down above $\sim 10^{10} M_{\mathrm{v}}$, where the "red sequence," occupied by massive galaxies having lower values of the SSFR, becomes more prominent. The importance of the gaseous component is reflected in the Kennicutt-Schmidt law, which relates the gas column density $\left(\Sigma_{\mathrm{gas}}\right)$ to the SFR surface density $\left(\Sigma_{\mathrm{SFR}}\right)$.

\footnotetext{
* Based on observations made with the Arecibo Observatory and the NASA Galaxy Evolution Explorer (GALEX). The Arecibo Observatory is operated by SRI International under a cooperative agreement with the National Science Foundation (AST-1100968), and in alliance with Ana G. Méndez-Universidad Metropolitana and the Universities Space Research Association. GALEX is operated for NASA by the California Institute of Technology under NASA contract NAS5-98034.
}

A super-linear slope is sometimes reached, e.g., $\sim 1.4$ as in Kennicutt (1998b), indicating that the SF efficiency is higher in regions of higher gas surface density. However, the empirical relations among galaxy properties that are derived from such surveys apply to the galaxy populations that dominate them, typically the more massive and luminous galaxies. The same relations may not apply to dwarf or low surface brightness (LSB) galaxies. Of particular relevance to this work, in such objects the environment where SF occurs may be quite different.

Compared to the optically bright and massive systems that dominate the SDSS, gas-rich dwarf galaxies are often underrepresented in samples selected by stellar mass. Often the optical emission arising in such systems is very blue, patchy, and of very LSB or small in extent. However, gas-rich, low-mass, low-metallicity, low optical surface brightness galaxies are important to the study of SF because the processes by which gas is converted into stars within such systems may mimic those that occurred in the early universe. As the most chemically unevolved systems within the present-day galaxy population, the faintest dwarfs represent unique laboratories for understanding $\mathrm{SF}$ and galaxy evolution in extreme environments, that is, in regimes of low metallicity, low dust content, low pressure, low shear, and low escape velocity (Begum et al. 2008).

Several recent works suggest that the SF in dwarf galaxies may proceed quite differently from that in large spirals. Based on a sample of very local dwarf irregular galaxies, the Faint Irregular Galaxies GMRT Survey (FIGGS; Begum et al. 2008; Roychowdhury et al. 2009) found a lower average $\Sigma_{\text {SFR }}$ than would be expected from the Kennicutt-Schmidt law (Kennicutt 1998b). Moreover, no threshold density is observed below which 
SF is completely turned off. Recently, Lee et al. (2007) explored the distribution of the SSFR against absolute magnitude for a complete sample of 300 star-forming galaxies within $11 \mathrm{Mpc}$ of the Milky Way, from the $11 \mathrm{Mpc}$ H $\alpha$ UV Galaxy Survey (11HUGS). In addition to confirming the transition in SF activity at the high-mass end, those authors found a second transition, with low-luminosity dwarf galaxies $\left(M_{B}-15\right)$ having a very large spread in their SSFRs. This second transition suggests that the star-forming behavior may be distinct at the lowest mass range. After showing that other potential drivers are not able to explain the magnitude of observed systematics, Lee et al. (2009a) suggest that the overprediction of the SFR by the UV flux compared to that estimated from $\mathrm{H} \alpha$ in dwarf systems is consistent with an initial mass function (IMF) deficient in the most massive stars. However, those authors also point out that it is possible that some combination of effects may conspire to produce the observed trend, and thus the requirement of systematic variations in the IMF can be avoided.

The 11HUGS sample is complete in $\mathrm{Hi}$ mass only above $2 \times 10^{8} M_{\mathrm{U}}$, becoming rapidly incomplete at smaller $\mathrm{Hi}$ masses. To develop further the current understanding of how the gas supply regulates SF in the lowest mass systems, a larger sample of extreme dwarf galaxies is needed. Making use of the Arecibo $L$-band Feed Array (ALFA), the ongoing Arecibo Legacy Fast ALFA (ALFALFA) extragalactic $\mathrm{Hi}$ line survey is specially designed to identify low-mass, gas-rich objects in the local universe (Giovanelli et al. 2005). Because of its combination

of wide areal coverage, sensitivity, and velocity resolution, ALFALFA has already detected more than 400 galaxies with $\mathrm{Hi}$ masses $M_{\mathrm{Hi}}<10^{8} M_{\mathrm{U}}$ (Haynes et al. 2011). While SF is more directly linked to the molecular interstellar component, the detection of $\mathrm{CO}$ in low-metallicity dwarfs is difficult (Leroy et al. 2005, and references therein), suggesting further that $\mathrm{CO}$ no longer traces $\mathrm{H}_{2}$ well. Furthermore, in many gas-rich dwarf galaxies, the $\mathrm{H} \mathrm{i}$ component dominates both the gas and the baryonic mass (e.g., Leroy et al. 2007). The combination of $\mathrm{H}$ i parameters from ALFALFA with complementary multiwavelength data contributed by SDSS and GALEX provides an ideal data set to investigate the abundance and distribution of gas-rich dwarfs and to explore the relations among their gas content, stellar populations, and SF properties.

This paper is organized as follows. In Section 2, we define our $\mathrm{H}$ i-selected sample and present its basic gas properties. In Section 3, we present the supplementary SDSS and GALEX data, especially our re-processed UV photometry. The directly measured colors and selected spectroscopic behavior are also briefly examined. In Section 4, we describe how we utilize spectral energy distribution (SED) fitting techniques to obtain physical parameters for the dwarfs, e.g., $M_{*}$ and the dustextinction-corrected SFR. We discuss in Section 5 the relations between gas, star, and SF in dwarfs, and how they compare to the overall ALFALFA $\mathrm{H}$ i-selected population. A summary is presented in Section 6.

All the distance-dependent quantities in this work
are com-
ated assuming $\Omega .3, X=0.7$, and $H_{0}=70 \mathrm{~km} \mathrm{~s}^{-1} \mathrm{Mpc}^{-1}$, and a Chabrier (2003) IMF is adopted.

\section{SAMPLE SELECTION}

In this section, we use the $40 \%$ ALFALFA catalog ( $\alpha .40$; Haynes et al. 2011) to define two $\mathrm{H} \mathrm{i}$-selected dwarf galaxy samples, one of which is complete in $\mathrm{H}$ i mass and velocity width (s-com). The second sample (s-sup) is less restrictive in those parameters but supplements the first through the availability of deeper GALEX NUV/FUV observations. We discuss here the selection of these two samples and their H i properties. In Section 4.1, we will define a third sample $s$-sed as a subset of dwarfs among the s-com and s-sup samples.

\subsection{The ALFALFA-SDSS Parent Sample}

Begun in 2005, the ALFALFA survey has been using the seven-beam ALFA receiver to conduct a blind search for $\mathrm{Hi}$ sources with $c z<18,000 \mathrm{~km} \mathrm{~s}^{-1}$ over $7000 \mathrm{deg}^{2}$ of high galactic latitude sky (Giovanelli et al. 2005). The targeted regions cover the sky visible to Arecibo $0<$ decl. $<+36$ in both the spring $\left(07^{\mathrm{h}} 30^{\mathrm{m}}<\right.$ R.A. $\left.<16^{\mathrm{h}} 30^{\mathrm{m}}\right)$ and fall $\left(22^{\mathrm{h}} 00^{\mathrm{m}}<\right.$ R.A. $<03^{\mathrm{h}} 00^{\mathrm{m}}$ ) night sky. With a median $c z$ of $\sim 8200 \mathrm{~km} \mathrm{~s}^{-1}$, ALFALFA for the first time samples the $\mathrm{H}$ i population over a cosmologically fair volume and is expected to detect $\sim 30,000$ extragalactic $\mathrm{Hi}$-line sources out to redshifts of $z \sim 0.06$. As a second-generation wide-area Hi survey, ALFALFA is designed to greatly improve on the $\mathrm{Hi}$ census derived from

previous results. For example, ALFALFA is eight times more sensitive than the H i Parkes All-Sky Survey (HIPASS; Barnes et al. 2001), with four times the angular resolution and three times the velocity resolution, all of which are essential to the discovery of the lowest $\mathrm{H}$ i mass objects. In particular, HIPASS detected only 11 objects with $M_{\mathrm{Hi}}<10^{7.5} M_{\mathrm{v}}$ (Zwaan et al. 2005), whereas ALFALFA is detecting hundreds

mass systems. For example, at the distance of the Virgo Cluster, ALFALFA is sensitive down to $\sim 3 \times 10^{7} M_{0}$ for sources with $\mathrm{S} / \mathrm{N} \sim 6.5$ (Giovanelli et al. 2007; Haynes et al. 2011). In addition, $\mathrm{Hi}$ source positions derived from ALFALFA can be determined with a median accuracy of about $20^{11}$ (see Equation (1) of Haynes et al. 2011), allowing the identification of optical/ UV counterparts for the vast majority of $\mathrm{H}$ i sources without the need for follow-up synthesis mapping. While source confusion within the 3.5 beam can affect sources at large distance, it has little effect on the identification of nearby dwarfs, except when they are located in close proximity to giant neighbors.

The current ALFALFA survey, " $\alpha .40$," covers $\sim 40 \%$ of the final survey area and includes a catalog of $15855 \mathrm{Hi}$ sources, 15041 of which are extragalactic (Haynes et al. 2011). The remainder have no optical counterparts (OCs) and lie at low velocities that are consistent with Galactic phenomena, e.g., as high-velocity clouds (HVCs). ALFALFA H i detections are further categorized by source reliability: Code 1 sources are reliable extragalactic detections with high signal-to-noise ratio (S/N 6.5), while Code 2 sources, also known as "priors," have lower $\mathrm{S} / \mathrm{N}(4.5 \diamond \mathrm{S} / \mathrm{N} \rightarrow 6.5)$ but coincide with an $\mathrm{OC}$ of known optical redshift matching the $\mathrm{H}$ i measurement. The HVCs are identified as Code 9 objects. Further details are given in Haynes et al. (2011).

$\mathrm{Hi}$ masses in units of solar mass are obtained from the relation $M_{\mathrm{Hi}}=2.356 \times 10^{3} D^{2} S_{\text {int }}$, where $D$ is the distance in Mpc and $S_{\text {int }}$ is the integrated $\mathrm{Hi}$ line flux density in units of $\mathrm{Jy} \mathrm{km} \mathrm{s}{ }^{-1}$. In the local universe, distance determinations suffer significantly from the uncertainty introduced by a galaxy's peculiar velocity. In order to minimize the $\mathrm{Hi}$ mass error introduced by the uncertainty in distances, we adopt a peculiar velocity flow model that incorporates both primary distances available from the literature and secondary distances derived from the SFI++ survey (Springob et al. 2007). The flow model derived by Masters (2005) is adopted for galaxies with $c z_{\mathrm{CMB}}<$ $6000 \mathrm{~km} \mathrm{~s}^{-1}$, while distances for more distant objects are derived from redshifts in the CMB rest frame. Primary distances are assigned to individual galaxies wherever available from the 
literature, and following the method discussed in Springob et al. (2007), galaxies identified as members of groups and clusters are placed at the distance to their assigned hierarchical unit. We have been conservative in ambiguous cases, assigning larger distances where a choice is given to avoid the inclusion of higher mass galaxies in the present analysis.

All of the spring sky coverage of ALFALFA and part of the fall sky survey region overlap the footprint of the SDSS Legacy Survey, thereby allowing a direct cross-match of the two. As part of the ALFALFA catalog production process, the $\mathrm{H}$ i detections have been cross-matched to SDSS DR7 photometric objects for 12,470 of the $\alpha .40 \mathrm{Hi}$ detections (Haynes et al. 2011). Through SED fitting to the five SDSS photometric bands (see Section 4.1), we are able to derive additional basic properties of the full $\alpha .40$-SDSS $\mathrm{H}$ i-selected parent sample. A more detailed discussion of the $\alpha .40$-SDSS-GALEX sample in general, as well as the selection effects characteristic of the $\alpha .40$ survey, will be presented in S. Huang et al. (2012, in preparation). As discussed in Haynes et al. (2011), the identification of OCs to the $\alpha .40 \mathrm{H} \mathrm{i}$ sources and the cross-match to the SDSS DR7 is not a perfect process; in individual cases, the wrong counterpart may have been selected, the SDSS photometry may be bad, etc. However, the cross-match with the SDSS DR7 allows us to make a first statistical study of the relationships between gas, stars, and SF and provides us with a parent sample of gas-rich galaxies within which we can explore the distinctiveness of the lowest $\mathrm{H}$ i mass systems.

\subsection{A Complete Hi-selected Dwarf Sample}

The studies that infer global properties derived from the SDSS main galaxy catalog are highly biased against the inclusion of dwarf irregular galaxies because of the magnitude and surface brightness limits on the SDSS spectroscopic targets $(r \vartheta 17.77$ and $\left.\mu_{r, 50} \vartheta 23.0 \mathrm{mag} \operatorname{arcsec}^{-2}\right)$. Since $M_{\mathrm{H}} / L_{\text {opt }}$ increases with decreasing $L_{\text {opt }}, \mathrm{H}$ i-selected samples are more inclusive of star-forming galaxies than optical samples of similar depth. Because of their relatively young stellar populations and low dust contents, gas-rich dwarfs are typically blue and often patchy in optical appearance; at the same time, they are often extended and diffuse in $\mathrm{Hi}$. Since the cold gas is the fuel needed to sustain $\mathrm{SF}$, a blind $\mathrm{H}$ i survey of sufficient depth, like ALFALFA, is especially effective in identifying star-forming systems at the low-mass end and hence should offer a full census of starforming galaxies in the local universe.

In order to identify a sample of low-mass, gas-rich dwarfs, we have applied selection criteria to the $\alpha .40$ catalog as follows: (1) ALFALFA detection code $=1$ or 2 (reliable sources and priors, but no HVCs); (2) $\log M_{\mathrm{H}} \underline{i}_{1}<7.7$; (3) velocity width of the $\mathrm{Hi}$ line, $W_{50}<80 \mathrm{~km} \mathrm{~s}^{-1}$; (4) the optical images were visually inspected to eliminate the ones without OCs, those that appear to be more massive but $\mathrm{H}$ i-deficient galaxies. Following the detailed analysis of the $\mathrm{Hi}$ mass error in Martin et al. (2010) and Haynes et al. (2011), the $\log M_{\mathrm{H} \text { i }}$ error in the 7.5 bin is $\sim 0.2$ dex. Hence, requirement (2) ensures that we are unlikely to miss dwarfs with $\log M_{\mathrm{H} i}<7.5$ due to their $\mathrm{Hi}$ mass error. Criterion (3) helps to ensure that we include only truly low-mass systems. Based on criterion (4), two gas-poor face-on giant galaxies (UGC $7622=$ NGC 4469 and UGC $7718=$ NGC 4526) have been removed. Both have SDSS $r$-band absolute Petrosian magnitudes brighter than -18 and are early-type spirals situated in the Virgo Cluster; their $\mathrm{Hi}$ masses and velocity widths are unusually low, probably due to interaction within the cluster environment. Additionally, extragalactic $\mathrm{H}$ i sources without OCs (38 of them) are dropped. As discussed by Haynes et al. (2011), the majority of those are part of the extended $\mathrm{Hi}$ structures in the Leo region: the Leo Ring and Leo Triplet (Stierwalt et al. 2009) are similar fragments associated with nearby groups of galaxies. With these objects removed, the final complete ALFALFA dwarf galaxy sample, referred to hereafter as $s$-com, contains 176 galaxies.

The designation of "complete" for this $\mathrm{H}$ i-selected sample emphasizes that it is a complete subset of the $\alpha .40$ catalog. Because the ALFALFA sensitivity depends not only on the integrated flux but also on the profile width, there is no simple translation of a limiting flux to the lower limit on the $\mathrm{H}$ i mass, but the completeness can be well characterized (Haynes et al. 2011). Of particular relevance here, we note that, at the mean $W_{50}=36.8 \mathrm{~km} \mathrm{~s}^{-1}$ of the $s$-com galaxies, $\alpha .40$ is $90 \%$ complete to $\log M_{\mathrm{Hi}}=7.1$ and $25 \%$ complete to $\log M_{\mathrm{H}}=6.9$, within a distance of $11 \mathrm{Mpc}$. In comparison, the 11HUGS sample is complete in $\log M_{\mathrm{H}}$ i only to 8.3 (Lee et al. 2009b) within the same distance. While we include all the low $\mathrm{H}$ i mass detections in the $s$-com sample out to a distance of $\sim 30 \mathrm{Mpc}$, the $\alpha .40$ completeness limit at that distance is well above the $\mathrm{H} \mathrm{i}$ mass upper limit of the $s$-com sample, $\log M_{\mathrm{H}} \mathrm{i}=7$.7. Thus, the $s$-com sample is not complete in a volume-limited sense, but it does probe the extreme low $\mathrm{H}$ i mass tail of the $\alpha .40$ catalog.

\subsection{Supplementary Dwarf Galaxies with GALEX Data}

Since ALFALFA is an ongoing survey, its catalog of $\mathrm{H}$ i detections continues to grow with time. Similarly, the simultaneous undertaking of the GALEX satellite mission has provided some opportunity to obtain images in the NUV and FUV bands for early ALFALFA detections, at least until the GALEX FUV channel failure in 2009. Because exploration of the population of galaxies that define the low-mass end of the $\mathrm{H}$ i mass function (HIMF) has always been one of the main goals of ALFALFA, we proposed to obtain GALEX MIS (Medium Imaging Survey) level FUV and NUV observations of low $\mathrm{H}$ i mass targets, based on early releases of the ALFALFA catalog, in GALEX cycles 3, 4, and 5 (GI3-84, GI4-42, and GI5-2). As ALFALFA has progressed, the identification of the lowest $\mathrm{H}$ i mass population has likewise been an ongoing process, extending to lower $\mathrm{Hi}$ masses as its catalog of $\mathrm{H}$ i sources has grown. Hence, the complete $\alpha .40$ low-mass sample $s$-com as defined above is more restrictive in $\mathrm{H}$ i mass than our GALEX target dwarf galaxy lists, which were based on early ALFALFA catalogs. Fortunately, although the criteria for the GALEX target selection derived from the early ALFALFA catalogs were less restrictive in terms of $\mathrm{H} \mathrm{i}$ mass and velocity width, the ALFALFA-based GALEX targets are nonetheless of relatively low mass. Of the 77 galaxies for which we acquired GALEX FUV/NUV observations, 24 overlap with the strictly complete sample $s$-com. The remaining 53 galaxies have somewhat higher $\mathrm{Hi}$ masses (see discussion in Section 2.4); we refer to this supplementary sample as s-sup. While the $s$-sup sample is not complete in any sense, the availability of GALEX MIS-depth imaging in both FUV and NUV bands for its galaxies allows us to explore with better statistics the low $\mathrm{H}$ i mass systems so that we can test for trends (or thresholds) with $\mathrm{Hi}$ mass at the low-mass end of the HIMF.

\subsection{Hi Properties of the ALFALFA Dwarf Sample}

The combination of the two samples s-com and s-sup yields a final ALFALFA-selected set of 229 low $\mathrm{Hi}$ mass and lowvelocity width dwarf galaxies upon which we base the analysis presented here. Table 1 presents the relevant UV, SDSS, and $\mathrm{Hi}$ 
Table 1

Properties of Dwarf Sample

\begin{tabular}{|c|c|c|c|c|c|c|c|c|c|c|c|}
\hline AGC & $\begin{array}{l}\text { R.A. (J2000) } \\
\left(\begin{array}{c}\mathrm{h} \mathrm{m} \mathrm{s}) \\
(2)\end{array}\right.\end{array}$ & 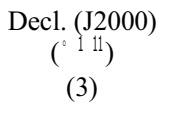 & $\begin{array}{c}m_{\mathrm{FUV}} \\
(\mathrm{mag}) \\
(4)\end{array}$ & $\begin{array}{c}m_{\mathrm{NUV}} \\
(\mathrm{mag}) \\
(5)\end{array}$ & $\begin{array}{c}r \\
(\mathrm{mag}) \\
(6)\end{array}$ & $\begin{array}{c}u-r \\
(\mathrm{mag}) \\
(7)\end{array}$ & $\begin{array}{l}\text { sFlag } \\
(8)\end{array}$ & $\begin{array}{c}D \\
(\mathrm{Mpc}) \\
(9)\end{array}$ & $\begin{array}{c}\log M_{\mathrm{H} \mathrm{i}} \\
(M \mathrm{O}) \\
(10)\end{array}$ & $\begin{array}{c}\log M_{*} \\
(M 0) \\
(11)\end{array}$ & $\begin{array}{c}\log \operatorname{SFR}(M \\
\left.\mathrm{Xg}^{-1}\right) \\
(12)\end{array}$ \\
\hline 102728* & 000021.4 & +310119 & $20.42(0.09)$ & $20.00(0.05)$ & $8.70(0.04)$ & $0.83(0.16)$ & par & $9.1(2.3)$ & $6.78(0.52)$ & $5.73(0.53)$ & -3.01( \\
\hline $748778^{*}$ & 000634.3 & +153039 & $19.78(0.09)$ & $19.65(0.05)$ & $18.14(0.05)$ & $0.81(0.19)$ & par & 4.6( & $6.36(1$ & $5.31(1.01)$ & -3.78 \\
\hline 102558 & 000704.6 & +270128 & $20.42(0.10)$ & $20.10(0.06)$ & $17.82(0$. & $0.96(1.31)$ & pbphot & 41.7( & $8.27(0$ & & \\
\hline 748779 & 000751.8 & +154518 & 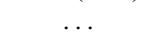 & & $18.86(0.05)$ & $1.08(0.29)$ & par & 12.4( & $7.09(0.3$ & & \\
\hline 101772 & 001108.6 & +14142 & & & $16.70(0$. & $0.98(0.0$ & & 11.7( & & & \\
\hline 102655 & 030 & +241759 & $\ldots$ & $18.90(0$. & 1 & & $\mathrm{ol}$ & . & & & \\
\hline $113753^{*}$ & 012102.1 & +260520 & $19.68(0.07)$ & $19.33(0.04)$ & $17.90(0.01)$ & $0.80(0$ & oly & 42.2( & $8.33(0.14)$ & $7.30(0.13)$ & $-1.80(0.72)$ \\
\hline 114027 & 013441.7 & +143836 & .. & $\ldots$ & $17.52(0.02)$ & $0.33(0$. & domi & $10.1(2$ & $7.40(0.46)$ & & $\ldots$ \\
\hline 112503 & 013800.3 & +145858 & & & $16.29(0.01)$ & $1.24(0.04)$ & par & $10.2(2.3)$ & $7.14(0.46)$ & & \\
\hline 1171 & 013944.8 & +155358 & $18.21(0.03)$ & $17.74(0.01)$ & $\ldots$ & $\ldots$ & $\ldots$ & $7.3(1.5)$ & $7.42(0.41)$ & $\ldots$ & $\ldots$ \\
\hline
\end{tabular}

(This table is available in its entirety in machine-readable and Virtual Observatory (VO) forms in the online journal. A portion is shown here for guidance regarding its form and content.)

properties for them. The magnitudes given in Table 1 have been corrected for foreground reddening. Columns are as follows:

1. Column (1): ALFALFA catalog identifier (also known as the AGC number).

2. Columns (2) and (3): J2000 position of the OC assigned to the $\mathrm{H}$ i source.

3. Columns (4) and (5): the adopted FUV and NUV magnitudes, with their associated error, respectively, as derived via our reprocessing of the GALEX images (see Section 3.2).

4. Column (6): the $r$-band modelmag with its associated error, from the SDSS pipeline.

5. Column (7): the $u-r$ color with its associated error, from the SDSS pipeline.

6. Column (8): the SDSS code sFlag indicating the quality of SDSS photometry as defined in Section 3.3.

7. Column (9): the adopted distance with error, in Mpc.

8. Column (10): the logarithm of the $\mathrm{Hi}$ mass and its error, taken from the $\alpha .40$ catalog (Haynes et al. 2011).

9. Column (11): the logarithm of the stellar mass and its error, derived from SED fitting (see Section 4.2)

10. Column (12): the logarithm of the SFR and its error, in solar masses per year (see Section 4.5)

Figure 1 shows histograms of the recessional velocity $c z$, adopted distance, observed $\mathrm{H}$ i line width, and logarithm of the $\mathrm{H}$ i mass for the combined ALFALFA dwarf sample, with shaded areas identifying the complete sample, s-com, and the open areas indicating the additional s-sup galaxies. As expected since the lowest $\mathrm{H}$ i masses are detected only nearby, most galaxies lie within the Local Supercluster at $c z<3000 \mathrm{~km} \mathrm{~s}^{-1}$. The peak in the distance distribution at $16.7 \mathrm{Mpc}$ arises from the assignment of Virgo membership to a significant number of the dwarf galaxies. A cross-match with the VCC catalog (Binggeli et al. 1985) shows that there are 37 Virgo members belonging to the dwarf sample defined here ( 35 in s-com and 2 in s-sup). The dashed vertical line in the $\mathrm{Hi}$ line width histogram corresponds to the adopted line width cutoff, $W_{50}<80 \mathrm{~km} \mathrm{~s}^{-1}$. The mean uncertainty on the line width measurement is $6.7 \mathrm{~km} \mathrm{~s}^{-1}$. Note that only one galaxy in the $s$-com sample is included in the last bin below this cutoff, suggesting that the low $\mathrm{H} \mathrm{i}$ mass and narrow line width criteria are consistent: we are not missing a population of high line width but still low-mass dwarfs because our line width cutoff is set to be too narrow, and the low $\mathrm{Hi}$ mass criterion is more important than the narrow line width one in the definition of the $s$-com sample. In agreement with this point, a quick check of the s-sup galaxies reveals the fact that they all have $\log M_{\mathrm{Hi}}>7.7$, although by the definition of the $s$-com subset, it is possible that galaxies in the s-sup sample could have $\log M_{\mathrm{H} \mathrm{i}}<7.7$ but $W_{50}>80 \mathrm{~km} \mathrm{~s}^{-1}$. In other words, the supplemental sample s-sup does not include low-mass dwarf galaxies that are excluded from the $s$-com one through the restriction to the velocity width, $W_{50}<80 \mathrm{~km} \mathrm{~s}^{-1}$. As anticipated, galaxies in the s-sup sample span a wider range of $W_{50}$ and distance than the stricter low $\mathrm{H} \mathrm{i}$ mass sample. In the histogram of $\log M_{\mathrm{H}}$, the low-mass tail extends to $\log M_{\mathrm{Hi}} \sim 6$; the sharp edge at $\log M_{\mathrm{Hi}}=7.7$ (our upper limit for s-com) reflects the fact that the s-sup galaxies represent only a small subset of the $\alpha .40$ galaxies in the higher H i mass range. Figure 2 of Haynes et al. (2011) shows the similar distributions for the full $\alpha .40$ catalog.

Previous studies of dwarf galaxies have focused mainly on optically selected samples and contain relatively few objects with $\log M_{\mathrm{H}}<$ 7.7. For example, the FIGGS sample of Begum et al. (2008) contains only 41 galaxies with $\log M_{\mathrm{H} \mathrm{i}}<7.7$, compared to 176 in the ALFALFA $s$-com sample. The majority of the FIGGS targets lie within $8 \mathrm{Mpc}$ and have been selected from existing optical surveys. Similarly, the volume-limited 11HUGS sample is complete in $M_{\mathrm{H} \mathrm{i}}$ to $2 \times 10^{8} M^{\mathrm{U}}$ (Lee et al. 2009b). In fact, only seven of the $s$-com plus $s$-sup galaxies are included in the 11HUGS catalog; at the same time, 54 of the ALFALFA dwarfs lie at distances of less than $11 \mathrm{Mpc}$. Figure 2 shows a Spaenhauer plot of $\mathrm{Hi}$ mass versus distance for the ALFALFA dwarfs, with filled circles denoting the $s$-com members. The lower edge of the distribution represents the ALFALFA sensitivity limit (Haynes et al. 2011). Compared to the similar plot in Figure 1 from Lee et al. (2009b), the distribution of ALFALFA dwarfs is shifted toward the lower $\mathrm{H}$ i mass range, just where the deviation of the UV-based SFR from that inferred from $\mathrm{H} \alpha$ is more likely to show up (Lee et al. 2009a). Benefiting from its improved sensitivity and angular and spectral resolution, the ALFALFA survey catalog allows us to draw a statistically significant sample of the lowest $\mathrm{H} \mathrm{i}$ mass galaxies in the local universe.

\section{DATA}

In this section, we describe the GALEX and SDSS data sets used, including reprocessed UV photometry from GALEX. In addition, the UV-to-optical color and emission-line diagnostics critical to the appraisal of SF in the dwarf galaxies are discussed. 

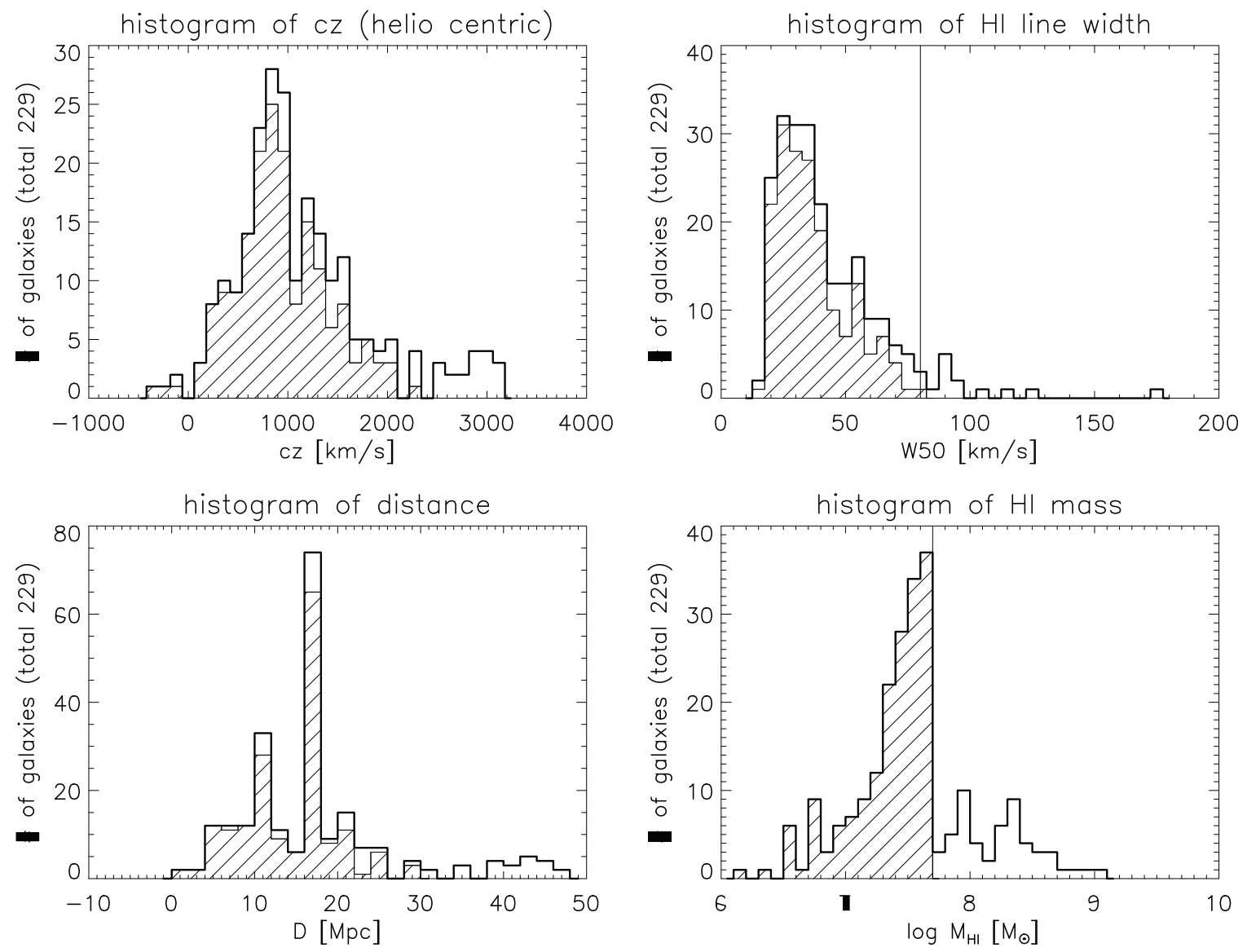

Figure 1. Hi properties of the full ALFALFA dwarf sample. In all panels, the shaded area identifies the galaxies in the complete $\mathrm{Hi}$ mass/velocity width-limited sample $s$-com $($ total $=176$ ), while the unfilled area represents the additional galaxies with $G A L E X$ photometry in the $s$-sup sample (total $=53$ ). Top left: the distribution of $\mathrm{Hi}$ line recessional velocity $c z_{\mathrm{Hi}}$. Top right: the distribution of $\mathrm{Hi}$ line velocity width $W_{50}$. The dashed vertical line corresponds to the $W_{50}$ cutoff $\left(W_{50}<\right.$ $80 \mathrm{~km} \mathrm{~s}^{-1}$ ) imposed on the s-com sample. Bottom left: the distribution of adopted distance to the ALFALFA dwarfs. The peak in the distribution at $16.7 \mathrm{Mpc}$ reflects the assignment of 37 galaxies to membership in the Virgo Cluster. Bottom right: the distribution of $\mathrm{Hi}$ mass. The dashed vertical line corresponds to the $M_{\mathrm{H}}$ i cutoff $\left(M_{\mathrm{H}}<10^{7.7} M_{\mathrm{U}}\right)$ imposed on the $s$-com sample.

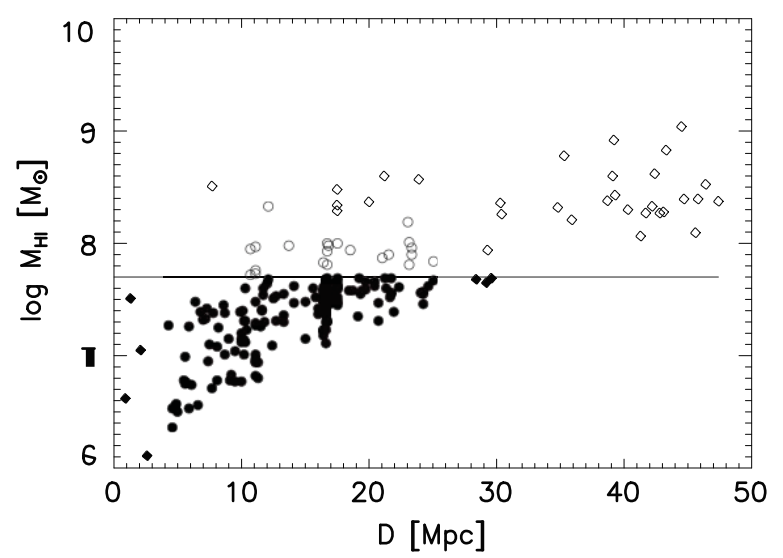

Figure 2. Spaenhauer diagram showing $\mathrm{H}$ i mass vs. distance for the ALFALFA dwarf galaxy sample. Filled circles denote the lowest H i mass $s$-com members, whereas open ones identify the supplementary s-sup galaxies. The horizontal dashed line corresponds to the $M_{\mathrm{Hi}}$ cutoff at $M_{\mathrm{Hi}}=10^{7.7} M_{\mathrm{U}}$ imposed on the s-com sample.

\subsection{Targeted GALEX Observations}

In dusty starbursting galaxies, a dominant fraction of the UV emission may be obscured by dust and reprocessed at FIR wavelengths (Treyer et al. 2007). In red sequence galaxies, older evolved stars make significant contributions to the UV luminosity (Wyder et al. 2007). However, the ALFALFA- selected dwarf galaxies are likely to suffer less from extinction, and their young stellar populations contribute the bulk of the UV light. The FUV luminosity $L_{\mathrm{FUV}}$ is generally thought to give the most robust measure of the SFR in individual galaxies with low total SFRs and low dust attenuation. Dwarfs are known to be low in metallicity and dust content, so that IR indicators of the SFR, which are calibrated via massive spirals, may be less reliable. In contrast to $\mathrm{H} \alpha$ emission, FUV photons primarily originate in the more abundant and relatively longer lived population of B-stars so that the FUV flux is not as vulnerable to stochastic effects. Furthermore, FUV photons are emitted directly from the stellar photospheres and thus do not suffer from possible uncertainties in the photoionization of the gas in low-density media (Lee et al. 2011).

In order to explore the UV properties of the faint and LSB galaxies in the ALFALFA dwarfs, we examined all available moderate-exposure (MIS-depth) GALEX images coincident with them, including both ones from our own GI programs and others available in the GALEX archive. GALEX simultaneously imaged the sky in the FUV (effective wavelength of $1516 \AA$ ) and NUV (effective wavelength of $2267 \AA$ ), with a circular field of view of $\sim 1.2$ in diameter (Morrissey et al. 2007). The images were processed through the GALEX pipeline, and the intensity maps with a 11.5 pixel scale were retrieved. With typical exposure times of $\sim 1500 \mathrm{~s}$, the images reach limiting magnitudes of $\sim 22.7 \mathrm{mag}$ in both the FUV and NUV, 
corresponding to surface brightness limits of $\sim 27.5 \mathrm{mag} \mathrm{arcsec}^{-1}$ or an SFR of $\sim 10^{-3} M_{\cup} \mathrm{yr}^{-1} \mathrm{kpc}^{-2}$ (Lee et al. 2011). As discussed in Section 2, all 53 s-sup galaxies were included in our GALEX GI programs, though five of them were only observed in the NUV due to the failure of the FUV detector. Since the final $s$-com sample was extracted from the $\alpha .40$ catalog after the FUV channel was completely turned off, we also searched the $G A L E X$ archive for any additional MIS-depth images with adequate coverage of ALFALFA dwarf galaxies. Among the final ALFALFA-GALEX/MIS sample of $77 s$-com galaxies, seven were observed in the NUV only.

Using a standard ellipse fitting extraction of the magnitudes (GALPHOT; see below), 67 of the 70 extreme dwarfs in the $s$-com sample are clearly detected in the FUV band. Two of the remaining three are extremely faint and LSB in the FUV, but magnitudes are still measurable in concentric apertures (ELPHOT; see below). Furthermore, all of the sources in the higher $\mathrm{Hi}$ mass supplementary s-sup sample that have FUV images are detected in FUV. Thus, only one out of the 118 dwarfs in the combined $s$-com plus $s$-sup sample with FUV MIS level images is a non-detection. A similarly high detection rate was found by Lee et al. (2011). Only 22 of the 390 galaxies in their 11HUGS sample observed by GALEX were not detected in FUV. About half of these are galaxies classified as faint dwarf ellipticals/spheroidals ( $\mathrm{dE} / \mathrm{dSph})$ and lack any evidence of recent SF; nearly all the others were found in images of exposure times less than $200 \mathrm{~s}$. Those authors concluded that, despite the variable, episodic, or bursty SFHs of dwarfs, the fluctuations in the SFR do not go to zero on timescales comparable to the lifetimes of UV-emitting stars ( 100 Myr). Furthermore, Lee et al. (2011) propose the need to examine the possible complete cessation of SF in low-luminosity systems via an $\mathrm{H} \mathrm{i-}$ selected sample probing masses down to $10^{7} M_{U}$, exactly what the ALFALFA s-com sample is. The presence of $\mathrm{Hi}$ selects against the very gas-poor $\mathrm{dE} / \mathrm{dSph}$ population, so our even higher FUV detection rate is not unexpected. This result clearly suggests that virtually all $\mathrm{H}$ i-bearing dwarf galaxies exhibit some level of recent SF.

\subsection{GALEX Photometry}

Because we rely mainly on the FUV luminosity to infer SFRs, it is essential to obtain accurate FUV photometry. Given the typical faint, LSB, and patchy nature of the UV emission of dwarf galaxies, extra attention must be paid to the extraction of magnitudes. The standard GALEX pipeline, which is based on the SExtractor code (Bertin \& Arnouts 1996), suffers from shredding if multiple star-forming sites are resolved. It also suffers from blending if foreground stars or UV-bright background galaxies are viewed in projection with the target galaxy. Additionally, the background determination matters more than that in high surface brightness regions, since the background subtraction uncertainty, rather than the photon noise, dominates in LSB regions (Gil de Paz et al. 2007). For these reasons, we developed our own tools to perform the photometric extraction on the GALEX images of the ALFALFA dwarf galaxies.

For each of the target galaxies, we extracted a portion of the intensity map retrieved from the MAST Web site. Further reduction of the image was performed within the IRAF $^{5} / \mathrm{STSDAS}$

\footnotetext{
IRAF is distributed by the National Optical Astronomy Observatory, which is operated by the Association of Universities for Research in Astronomy, Inc., under cooperative agreement with the National Science Foundation.
}

environment using a set of scripts developed for previous $I$-band imaging surveys undertaken by our group, referred to as the GALPHOT package (Haynes et al. 1999), and appropriately modified to accommodate the GALEX images. Because the morphology of galaxies in the FUV is not necessarily the same as in the NUV, and because foreground stars are often much brighter in the NUV images, we elected to work on the two channels separately rather than adopt a single identical set of apertures.

A constant value associated with the sky background was subtracted using a procedure that allows the user to mark boxes that are free of bright stars and galaxies on each frame. The boxes are placed so that they surround the target galaxy but are far enough from it to avoid the influence of faint extended emission. In contrast to the circumstances applied to optical images, the UV sky is so dark that the pixel values in sky boxes follow a Poisson distribution rather than a Gaussian one. The subtracted sky value in the UV case is the mean intensity obtained within the sky boxes after iteratively clipping out the pixels whose values are more than $3 \sigma$ above the mean value, a process that removes the faint stars and galaxies within the sky boxes. Usually this process converges quickly after 1 or 2 clipping cycles.

To clean the regions over which the galaxy photometry is to be derived, we first used an automatic procedure to mask UV sources at least 2 galaxy radii away from its center and then masked by hand sources within 2 radii deemed to be unrelated to the galaxy itself. Starting from an initial guess marked by hand, elliptical surface brightness contours were then fitted to the cleaned images, using the STSDAS package ISOPHOTE, outward to the radius at which the fitting fails to converge, and inward to the seeing limit. This process yields the azimuthally averaged surface brightness profile, as well as the variation with semimajor axis of the ellipse centroid, its position angle, and ellipticity; these ellipses are then used later as the apertures for the photometric extraction and allow for the interpolation of masked regions. The disk portion of the surface brightness profile is then fit by a linear function, using an interactive procedure that allows specification of the inner and outer disk radii, as discussed by Giovanelli et al. (1994) and Haynes et al. (1999). Figures 3-6 illustrate examples of the isophotal fitting result for four representative cases. Selected SDSS and GALEX images of them are shown in Figure 7.

Figure 3 shows the result for AGC $110482=$ KK 13, a patchy dwarf galaxy whose FUV flux is dominated by two bright knots. The GALPHOT isophotes are centered on the brighter knot in the inner region, but the ellipse fitting treats the merged light from the two knots as a single disk at large radius. This process results in a second peak in the surface brightness profile, when the ellipses reach the center of the fainter knot, but at large radii, the surface brightness profile falls exponentially as expected, yielding a valid total magnitude.

This process of fitting the disk assumes that the surface brightness profile of dwarf irregulars follows an exponential falloff (Hunter et al. 2010) so that disk scale lengths can be determined. However, dwarfs sometimes show multiple disk components, with clearly different slopes in inner and outer regions. In such cases, we fit two linear functions to each portion individually and use the inner fit to determine the general disk properties, e.g., scale length, position angle, etc., whereas the outer fit is used to extrapolate the surface brightness profile beyond the outermost measured isophote. Among the 130 ALFALFA dwarfs observed in GALEX (12 with no FUV 
-GC110482
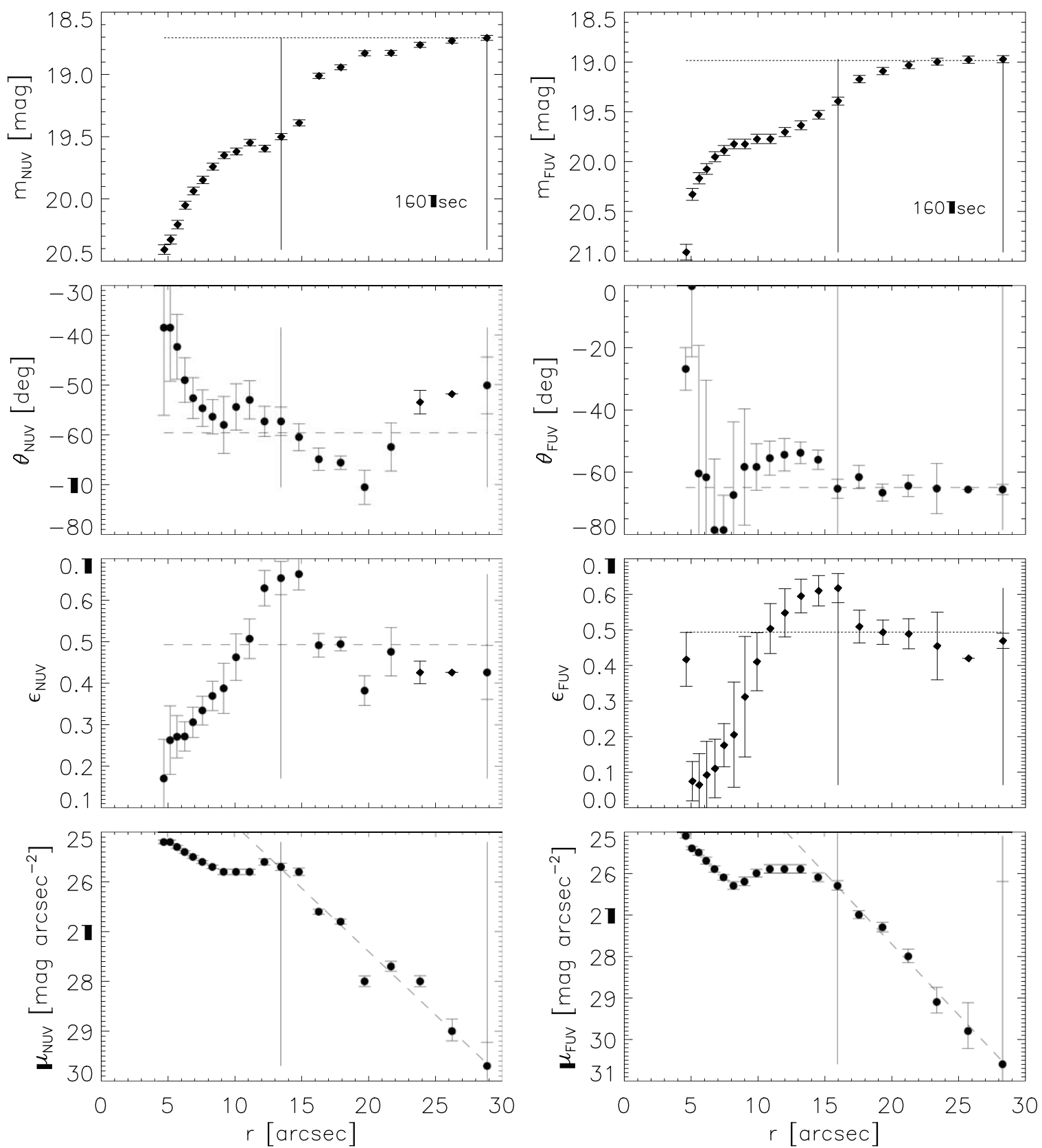

Figure 3. Example of the UV isophotal fitting result for AGC $110482=\mathrm{KK} 13$, a galaxy that shows star formation in two UV bright knots. The enclosed magnitude $(m)$, position angle $(\theta)$, ellipticity $(E=1-b / a)$, and surface brightness $(\mu)$ of the elliptical isophote as a function of the semimajor axis of the isophote $(r)$ are plotted in rows from top to bottom. Results for the NUV image are shown in the left panels, for the FUV image on the right. Numbers at the corners of each plot in the top row give the exposure times in each channel. The marked disk region is denoted by the vertical dash-dotted lines. Horizontal dashed lines represent the adopted magnitude $m_{8 r_{D}}$ (flux within $8 r_{D}$ ) in the $m$ plot, and final values determined from the mean of data points in the disk region in the $\theta$ and $E$ plots. Dashed lines in the $\mu$ plots show the linear fit to the light profile in the adopted disk region, assuming an exponential drop-off in surface brightness.

exposure), 28 have such double disks. The majority of those $(20 / 28)$ have shallower outer disks in the NUV (e.g., AGC 122212 in Figure 4 and its images in Figure 7), but 15/20 of them show no outer disk in the FUV (e.g., AGC 213796 in Figure 5 and images in Figure 7). Of the eight blue compact dwarfs (BCDs) studied by Hunter et al. (2010), five also have NUV double exponentials, all of which drop more shallowly in the outer disk. Those authors argue that the shallower outer profile represents the underlying stellar population, while the steeper inner profile is dominated by the centrally concentrated and intense recent SF. Consistent with this interpretation, the shallow outer disks are frequently not in evidence in the FUV. Further evidence for this scenario arises from the fact that the disk scale length is smaller in the FUV band compared to that seen in the NUV, again suggesting that recent $\mathrm{SF}$ is more centrally concentrated than is the overall stellar disk, i.e., the active star-forming region is shrinking.

On the other hand, of the 29 dwarf irregulars studied by Hunter et al. (2010), eight have clear double exponential profiles in the NUV; in only one of those is the outer exponential shallower than the inner one. Similarly, most of the ALFALFA dwarfs with steeper outer disks (7/8) belong to the higher $M_{\mathrm{H} \mathrm{i}}$ 
GC122212
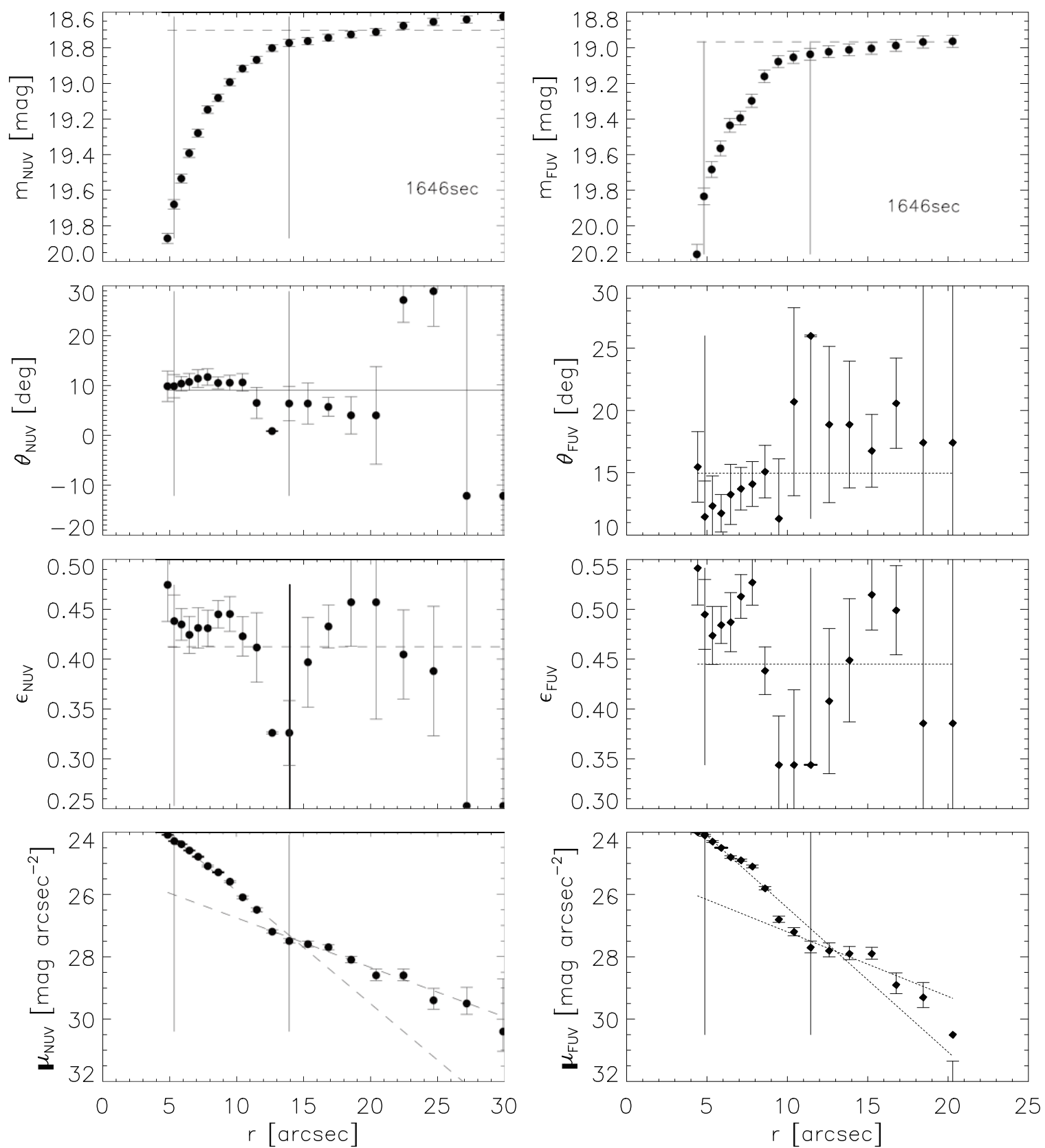

Figure 4. Example of the isophotal fitting result for AGC 122212 fit best by a combination of two disks. The symbol definition is the same as that in Figure 3 . This galaxy has a shallower outer disk in both the NUV and FUV images. Separate linear functions are fit to the inner and outer regions; the inner fit determines the disk scale length, and the outer fit is used for extrapolating the profile.

s-sup sample; it is possible that some may be more massive galaxies with more extinction in the inner regions. An example of this case is shown in Figure 6 for AGC $212824=$ KK 100 (see its images in Figure 7), which has the highest $M_{\mathrm{Hi}}$ in our sample. Another possible explanation for the apparent flattening of the UV profile was proposed in Boissier \& Prantzos (2000), namely, that the SFR in the inner disk has been higher than the infall of the gas, leading to a progressive consumption of gas toward the center. In the outer parts, however, SF is less efficient, and the infall of gas proceeds on longer timescales. As a result, the gas reservoir of the outer disk is not exhausted, and the shape of the exponential profile is preserved. The steeper outer disk may thus be more evident in the gas-rich dwarf irregulars, while

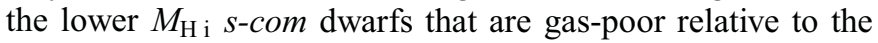

overall ALFALFA population (see Section 5.3) frequently show flatter outer UV profiles.

Once ellipses are fit, magnitudes are calculated using the IRAF routine POLYPHOT to measure the total flux within the ellipses and the disk fits are used to extrapolate beyond the measured isophotes. Following Haynes et al. (1999), several sets of magnitudes are recorded, including ones at fixed isophotal levels, partial magnitudes integrated to a certain number of disk scale lengths, and asymptotic magnitudes extrapolated to infinity. The extrapolation helps to recover the LSB outer emission below the sky level. While magnitudes extrapolated to infinity are adopted by the 11HUGS studies (Gil de Paz et al. 2007), we follow the discussion in Haynes et al. (1999) and adopt in this work a total magnitude computed at a radius of 


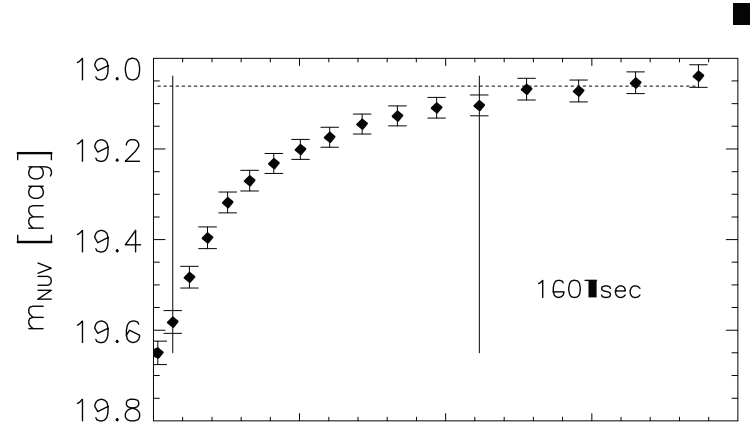

GC213】96
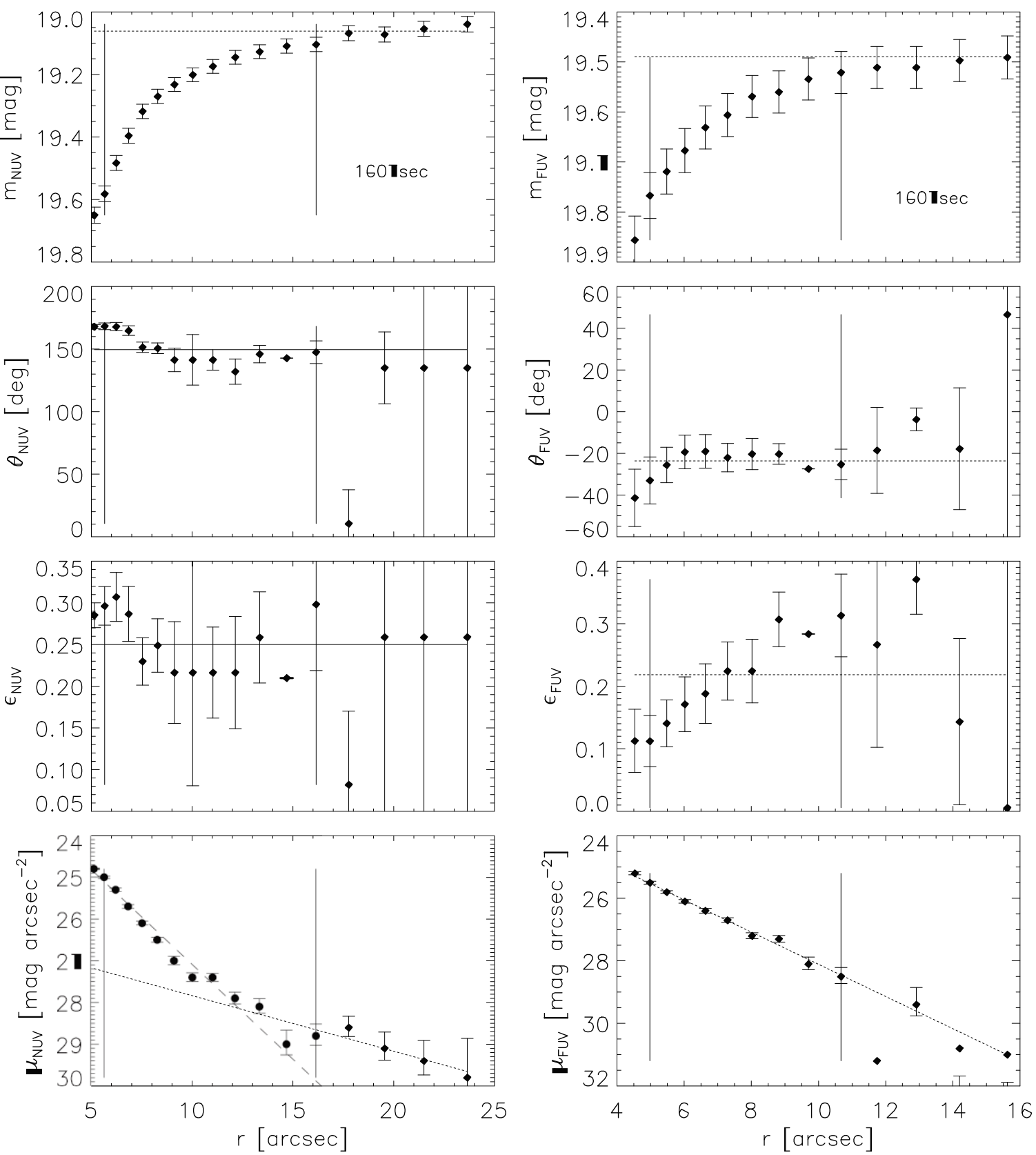

Figure 5. Example of the isophotal fitting result for AGC 213796. The symbol definition is the same as that in Figure 3 . This galaxy exhibits a two-disk structure only in the NUV, either because the emission in the FUV image is too weak to trace the outer disk or because the extended outer disk is not actively forming stars. The traceable FUV disk matches the inner NUV disk. Similar results are observed in a total of 15 galaxies that are assigned to this category.

eight disk scale lengths $8 r_{d}$. Note that for the majority of the galaxies, a radial extent of $8 r_{d}$ lies beyond the outermost radii marked as defining the disk region, except in a few cases in which the UV emission is compact and the image is particularly deep (e.g., AGC 225852 in the NUV and AGC 220609 in the FUV). To determine the error in the total magnitude, following Salim et al. (2007), we add the zero-point calibration errors of 0.052 (FUV) and 0.026 (NUV) mag to the Poisson errors, as well as the uncertainty in the determination of the sky level, which is formally the rms noise of the pixel values in the sky boxes after masking the sources contained within them. We do not account for other types of errors, e.g., the flat-fielding errors, which are improved in the products of the latest version of the GALEX pipeline (GR6).
Because of their extreme LSB in the UV, two galaxies, AGC $201970=$ LeG 18 and AGC $223913=$ VCC 1649 (see its images in Figure 7), fail to produce convergence of fitted isophotal ellipses. The former one is also LSB in the SDSS images, but AGC 223913, a dE/dSph in Virgo, is only faint in the UV (see Section 3.4 for more discussion). For these two objects, the GALEX pipeline extracts a handful of unmatched faint sources in the NUV and FUV at the location of the target galaxy. We assign concentric elliptical apertures with increasing semimajor axis by hand to extract a curve of growth and adopt their magnitudes from those measured in the outermost aperture.

Only one galaxy, AGC $220483=$ VCC 628 , is too faint in the FUV to yield a reliable magnitude; it is also undetected by the GALEX pipeline to a limiting magnitude $\sim 22.7 \mathrm{mag}$. It is a dwarf 


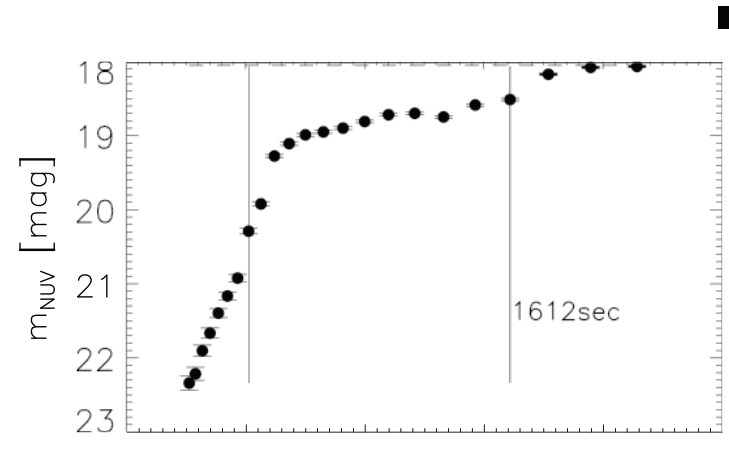

GC212824
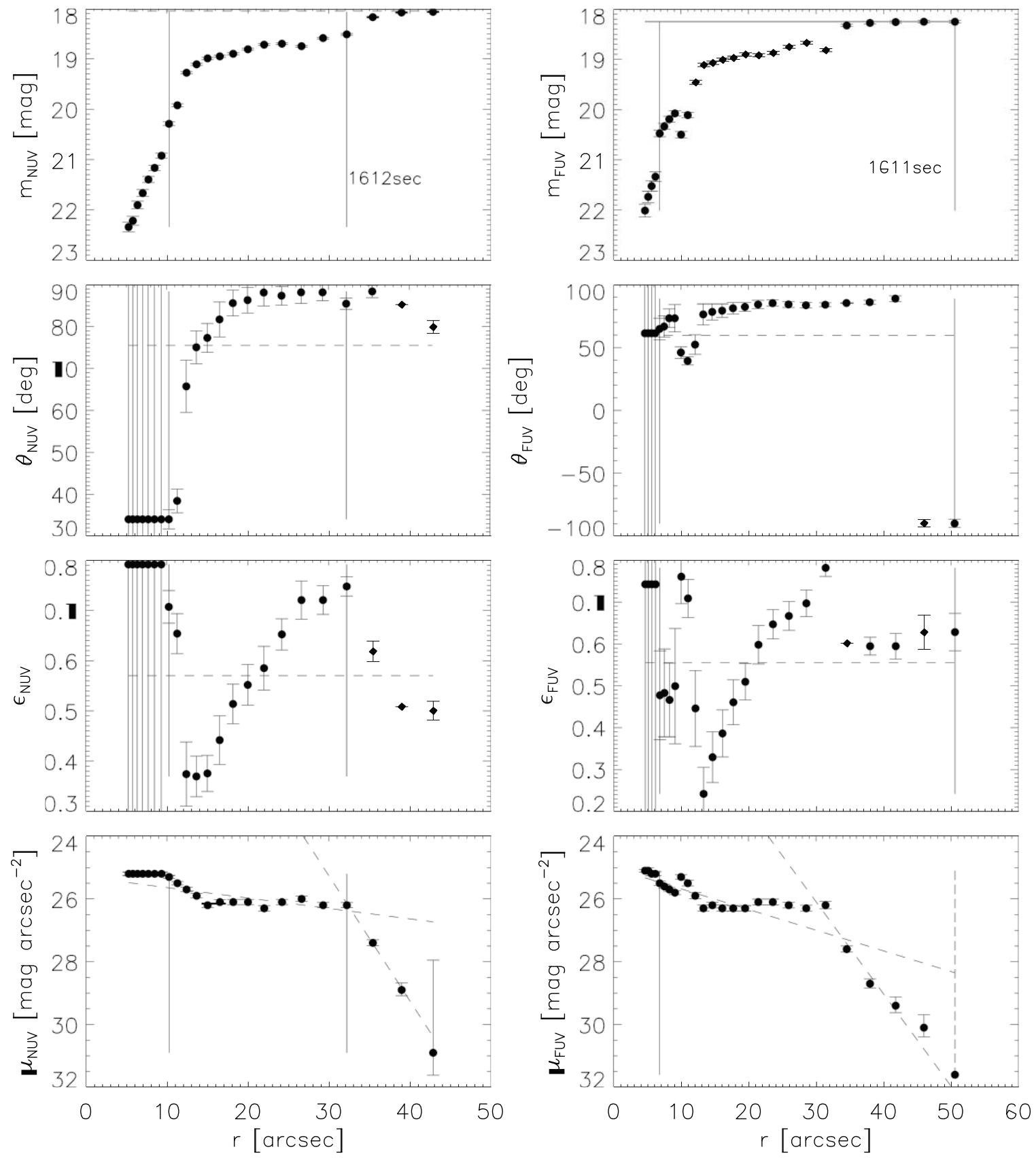

Figure 6. Example of the isophotal fitting result for AGC $212824=\mathrm{KK} 100$, which has an outer disk that is steeper than the inner one. The symbol definition is the same as that in Figure 3. This object has the highest $\mathrm{Hi}$ mass of any galaxy in the supplementary s-sup sample, $\log M_{\mathrm{H}} \mathrm{i}=9.04$. The flattening of the UV profile may be due to higher extinction in the center regions or alternatively to a higher efficiency of star formation in the inner disk.

irregular (Im) associated with the Virgo B Cluster but moving at very high velocity; it exhibits LSB also at optical wavelengths. It remains the lone object in our sample of ALFALFA dwarfs with MIS-depth GALEX FUV imaging to exhibit no traceable FUV emission.

A comparison between our GALPHOT/ELPHOT-derived magnitudes and the GALEX pipeline magnitudes (GR6) is shown in the upper row of Figure 8 for the NUV (left) and FUV (right) channels, respectively. The magnitude difference is defined as ( $\left.\operatorname{mag}_{\mathrm{GALPHOT}}-\mathrm{mag}_{\mathrm{GR} 6}\right)$. Filled and open circles denote the galaxies in the s-com and s-sup samples, respectively. In addition to AGC $220483=$ VCC 628, which was not detected in the FUV as discussed above, several galaxies are excluded from these plots because they were not detected by the GALEX pipeline in either one band or the other: AGC 205097 in the NUV (two NUV-bright stars are nearby), AGC 112505 in the NUV (blend with one nearby NUV-bright star and also very close to UGC $1176=$ DDO 13), AGC 223913 in the FUV $(=\mathrm{VCC} 1649, \mathrm{dE}$ in Virgo, LSB in the NUV, invisible in the FUV by eye; SDSS and GALEX images shown in Figure 7). In addition to these non-detections, several clear outliers are seen in Figure 8. The outliers in the FUV plot have a brighter magnitude obtained by the "GALPHOT" ellipse fitting than that measured by the GALEX pipeline. Close inspection shows that they are mostly extended irregulars with large disk scale lengths and patchy UV emission; they suffer from significant shredding 


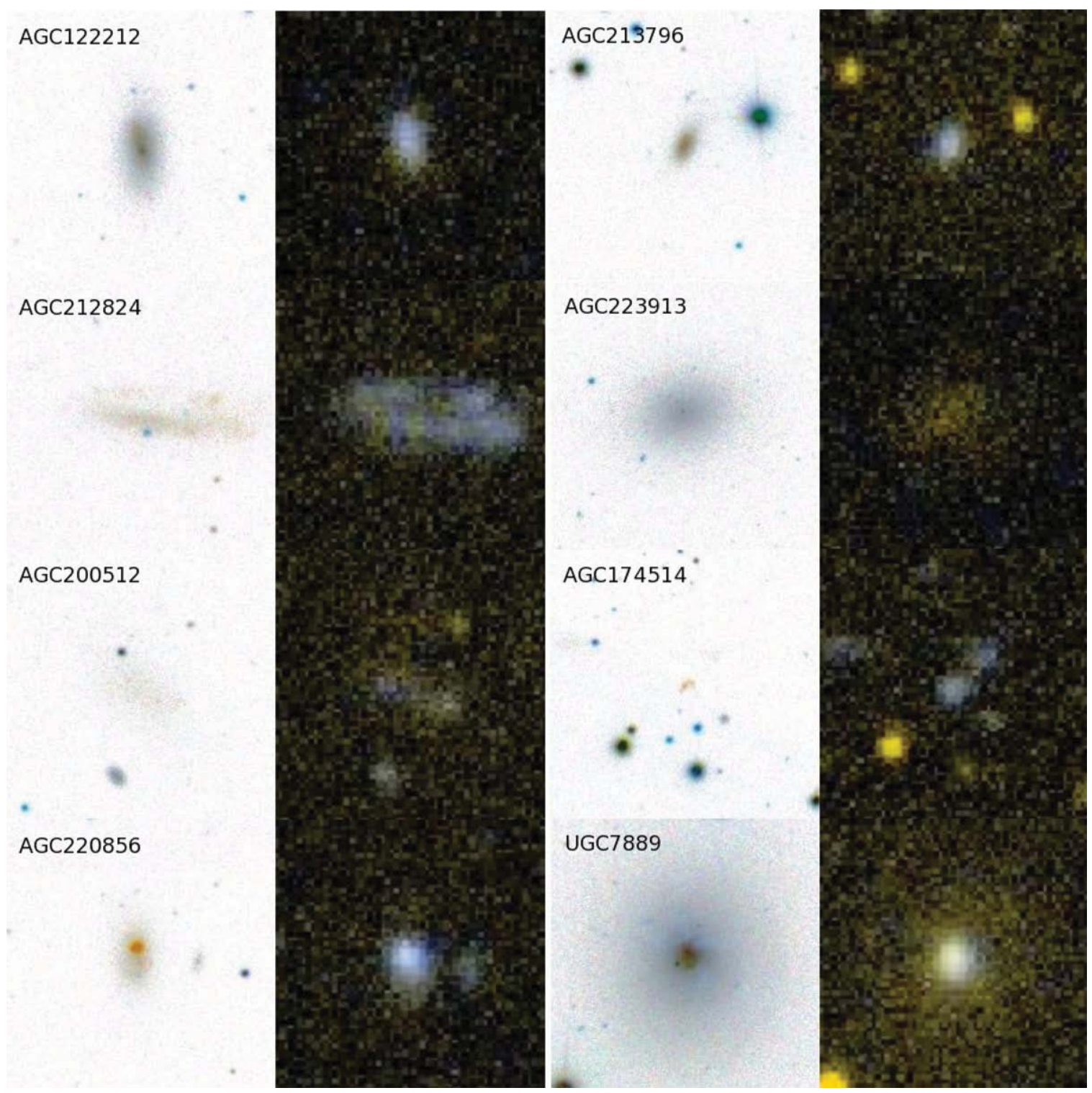

Figure 7. Gallery of ALFALFA dwarf galaxies discussed in the text. Inverted SDSS images, 1.5 on a side, are shown to the left of the GALEX images (not inverted) of the same galaxies. First row: AGC 122212 (left) and AGC 213796 (right) both belong to the s-com sample and have shallower outer disks in NUV. The latter has no FUV outer disk. Second row: AGC $212824=\mathrm{KK} 100$ (left) is patchy and has a steeper UV outer disk. It is also the most H i massive galaxy in the ALFALFA dwarf sample, belonging to the s-sup set. AGC $223913=$ VCC 1649 (right) is a dE/dSph in Virgo with the lowest SSFRs among the s-sed set (see its definition in Section 4.1), i.e., its SF is suppressed. Third row: AGC 200512 = LeG 6 (left) is LSB and thus is shredded by the GALEX pipeline measurement in FUV. AGC 174514 (right) has the highest $f_{\text {gas }}$ value and bluest $u-r$ color among the $s$-sed galaxies. Faint patches nearby may be associated with the same object. Bottom row: AGC $220856=$ VCC 1744 (left) and UGC $7889=$ NGC 4641 (right) are both BCDs in Virgo. The former suffers from serious emission-line contamination and has the highest $b$-parameter value among the $s$-sed objects.

(A color version of this figure is available in the online journal.)

by the GALEX pipeline, e.g., UGC 4415 and AGC 122206 in the NUV; AGC 200512 in the FUV (SDSS and GALEX images are shown in Figure 7); AGC 201970 in both the NUV and FUV. Several galaxies lie at such nearby distances that they have resolved H ii regions visible in the FUV (e.g., UGC 12613 with a distance of only $0.9 \mathrm{Mpc}$; UGC $5373=$ Sextans B at $1.3 \mathrm{Mpc}$ ). After excluding the non-detections and outliers that arise from blending or shredding by the pipeline process, the magnitudes we derive are in reasonable agreement with the pipeline magnitudes. The improved GR6 pipeline magnitudes agree better with our results, especially in the NUV.

The bottom row of Figure 8 shows the same magnitude difference versus the disk scale length $r_{D}$ on a logarithmic scale. As evident in Figure 8, our photometric extraction technique, which is specifically designed to capture all of the LSB flux, yields brighter magnitudes for a significant number of galaxies. The median magnitude difference is -0.035 and $-0.073 \mathrm{mag}$ in the NUV and FUV, respectively. A weak trend is seen such that, as the galaxies become more extended (larger $r_{D}$ ), the GALEX pipeline misses the outer LSB UV emission and hence underestimates the true UV magnitude. For the remainder of this work, we use our own measurements of GALEX UV magnitudes, denoted as $m_{\mathrm{NUV}}$ and $m_{\mathrm{FUV}}$.

\subsection{SDSS Data}

In addition to the UV photometry from GALEX, we use optical data from the SDSS Data Release 7 (Abazajian et al. 2009). The 

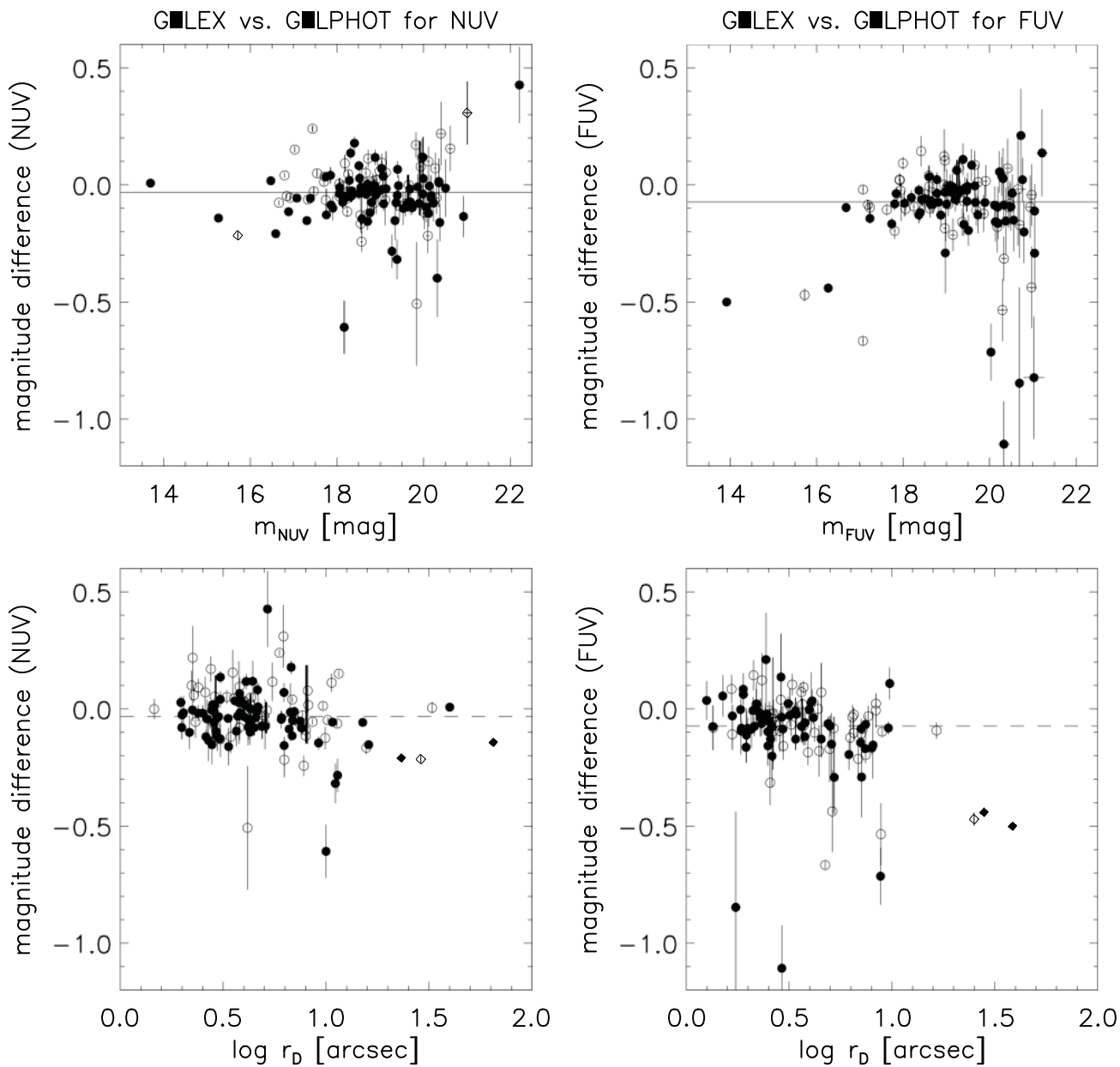

Figure 8. Comparison of the total UV magnitudes of the ALFALFA dwarf sample obtained by using the GALPHOT package with those derived from the GALEX pipeline. The magnitude difference is defined as $\left(\operatorname{mag}_{\mathrm{GALPHOT}}-\operatorname{mag}_{\mathrm{GR}}\right)$. Results for the NUV channel are shown in the left panel, for the FUV on the right. Filled circles denote the galaxies within the complete s-com sample, and open ones, the additional s-sup objects. The galaxies that are completely missed by the pipeline are excluded from these plots. Upper: the magnitude difference as a function of our measurements. In general, the GALPHOT photometry measures a brighter magnitude for a significant number of galaxies, especially in the FUV. Lower: the magnitude difference as a function of disk scale length $r_{D}$ on a logarithmic scale. A weak trend is seen that the pipeline becomes ineffective at detecting all emission in the more extended galaxies. Disk scale lengths are smaller on average in the FUV, i.e., the SFR is more centrally concentrated.

SDSS database provides photometry in five bands $(u, g, r, i, z)$ and spectroscopic follow-up for most galaxies with $r<17.77$. For the present work, we use the SDSS archival measurements to derive the properties of the underlying stellar population, but because of issues of shredding and blending similar to those found with the GALEX magnitudes, significant caution is applied. Given the higher resolution of the SDSS imaging and the higher density of bright stars, the problems associated with magnitude estimation are worse in the optical than in the UV. To check for such issues, we inspected visually each galaxy and the magnitudes derived from the SDSS pipeline and adopted the photometric properties associated with the best photometric cross match along with its reported quality code, sFlag (see below). As suggested by the SDSS team, modelmag magnitudes are adopted to derive stable colors while still capturing most of the total light.

Of the 229 galaxies in the $s$-com and s-sup samples, 24 lie outside of the SDSS Legacy Survey footprint; most of these are found in the fall sky region of ALFALFA $\left(22^{\mathrm{h}}<\right.$ R.A. $<3^{\mathrm{h}}$, $0<$ decl. $\left.<+36^{\circ}\right)$. It is well known that the SDSS photometric pipeline is optimized for small, high surface brightness objects (West et al. 2010), not the clumpy and LSB dwarf galaxies typical of our sample. In fact, of the 205 ALFALFA dwarfs in the SDSS sample, only 44 ALFALFA dwarfs are extracted
as single photometric objects by the SDSS pipeline (sFlag $=$ "oly" as given in Column (8) of Table 1). Since multiple peaks are usually evident in the light profiles of patchy dwarfs (the "parent" object), these are often de-blended by the standard pipeline into pieces (the "child" objects). Accurate magnitudes can be recovered in those cases where the "parent" object contains all (or nearly all) of the emission associated with the galaxy and is not blended with any other nearby objects. Fiftythree of the ALFALFA dwarfs belong to this category (sFlag = "par"); in these cases, the magnitudes of the associated parentID are adopted. For the remaining objects for which the "parent" objects still suffer from blending, we examined the magnitudes of each "child" object within the region of the target galaxy. According to West et al. (2010), roughly $75 \%$ of galaxies have more than $90 \%$ of their flux contained in the brightest child. We used the magnitudes of the brightest child where it is brighter than the second brightest child by at least 3 mag, implying that it contains the vast majority of the galaxy's flux. Fifty-five of the 205 ALFALFA-SDSS dwarfs fit this category (sFlag = "domi") so that the SDSS magnitudes contribute a satisfactory lower limit of the optical flux. The remaining 53 galaxies have photometry too uncertain to be used further, usually because the parent 


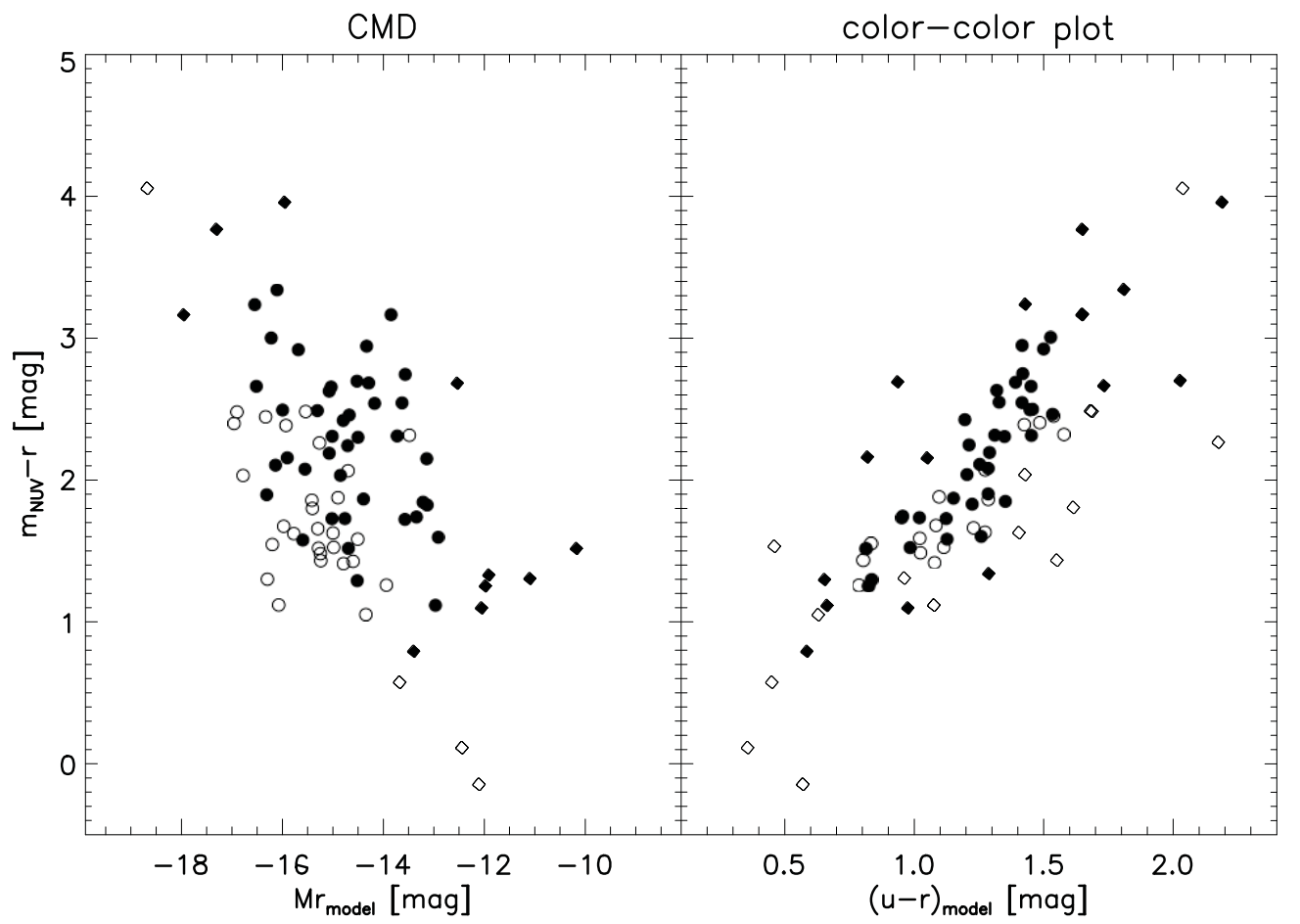

Figure 9. UV-to-optical colors of the ALFALFA dwarfs. Filled circles identify dwarfs in the lowest $\mathrm{H}$ i mass complete $s$-com sample, while open ones represent the additional $s$-sup galaxies. Left: color-magnitude diagram. Unsurprisingly, nearly all the ALFALFA dwarf galaxies are less luminous than $M_{r} "$ " -18 and lie below the blue cloud-red sequence divider quoted by Salim et al. (2007), $m_{\mathrm{NUV}}-r, S 4$. Right: color-color plot. Within the blue color range occupied by the sample overall, the $m_{\mathrm{NUV}}-r$ color is well correlated with the $u-r$ color, with the former probing a larger dynamical range, $\delta\left(m_{\mathrm{NUV}}-r\right) / \delta(u-r) \sim 2$.

object is blended with other sources or has no dominant child $($ sFlag $=$ "pbphot"). It is relevant to note that most are dropped for one of two specific reasons: either (1) because they are very patchy or (2) because they are contaminated by the presence of nearby or superposed stars. The latter reason has nothing to do with the galaxy itself, but the first is particularly common for this sample of dwarf galaxies. We checked the distribution of $\mathrm{H} \mathrm{i}$ properties for the excluded objects and find that they span the full range in distance, line width, and $\mathrm{Hi}$ mass evident in Figure 1, suggesting that the inclusion of a criterion of acceptable SDSS photometry introduces no extra bias in terms of $\mathrm{H}$ i parameters. Although we have not reprocessed the SDSS photometry as did West et al. (2010), the careful visual inspection technique applied here effectively avoids the shredding or blending of the SDSS magnitudes that are the main causes of the failure of the SDSS pipeline to deliver reliable photometry for diffuse, patchy, and/or LSB galaxies.

However, because of the typical faintness and LSB optical appearance of the ALFALFA dwarfs, the percentage of the photometric objects that are targeted for SDSS spectroscopy is quite low: only $114 / 205$ have a counterpart in the SDSS spectroscopic survey. This circumstance further validates the importance of $\mathrm{H} \mathrm{i}$ selection for deriving a complete sample of dwarfs in the local universe. Furthermore, only 101 of the 114 spectroscopic targets are included in the MPA-JHU DR7 release of SDSS spectral measurements (http://www.mpa-garching.mpg.de/SDSS/DR7/; Brinchmann et al. 2004), which contains reprocessed line flux, etc. We confirm that all 101 dwarfs are star-forming or low S/ $\mathrm{N}$ star-forming galaxies, following the classification in Brinchmann et al. (2004). In comparison with the SDSS-selected population, the $\mathrm{H}$ i-selected dwarf sample includes a much smaller representation of galaxies containing AGNs or classified as "non-star-forming"; this finding also implies that the SDSS magnitudes are more likely to be contaminated by line emis- sion. Similarly, a quick inspection shows that all the ALFALFA dwarf galaxies with SDSS $D_{n}(4000)$ measurements have a value below 1.6, the demarcation in the bimodal distribution derived in Kauffmann et al. (2003a). This result suggests that these dwarfs have been actively forming stars throughout their history.

\subsection{UV-to-optical Colors of the ALFALFA Dwarfs}

The global CMD is a powerful tool for the assessment of the basic properties of a sample of galaxies. The bimodal nature of the field galaxy CMD is well demonstrated with the large data sets available from recent large-scale galaxy surveys, in particular SDSS (Baldry et al. 2004). It is clear that galaxies separate into the "red sequence" of early-type galaxies that show little or no evidence of ongoing SF (corresponding to the low SSFR portion in the SSFR versus $M_{*}$ diagram) and the "blue cloud" of star-forming spirals (corresponding to the starforming sequence in that diagram). Compared to the traditional optically based CMDs, a CMD constructed from a UV-to-optical color provides a more powerful diagnostic (Wyder et al. 2007; Schiminovich et al. 2007; Salim et al. 2007). By contrasting the recent SF, as indicated by the UV light, to the total past SF, as indicated by the optical light, the UV-to-optical color provides a more concrete diagnostic of a galaxy's SFH (Salim et al. 2005).

To produce the CMD shown in Figure 9, the magnitudes given in Table 1 have been corrected for foreground reddening. For the SDSS bands, we adopted the pipeline extinction correction. For the $G A L E X$ bands, we used $E(B-V)$ values based on the FIR DIRBE maps of Schlegel et al. (1998), the Cardelli et al. (1989) extinction law with $R_{V}=A_{V} / E(B-V)=3.1$, and $A(\lambda) / E(B-V)=8.24$ for the FUV and 8.2 for the NUV bands, respectively, following Wyder et al. (2007). Of the 229 galaxies listed in Table 1, 51 belonging to the complete $s$-com sample 
and 32 to the higher $M_{\mathrm{H}}$ i supplementary s-sup set have both acceptable SDSS photometry (sFlag not "pbphot") and NUV magnitudes, making it possible to estimate a color $\left(m_{\mathrm{NUV}}-r\right)$. Their distribution in the combined UV-optical CMD is shown in the left panel of Figure 9, with filled circles denoting objects in the $s$-com sample and open ones representing the additional s-sup galaxies.

The first obvious difference of this work from most previous studies is the much fainter absolute magnitude range probed by the ALFALFA dwarfs. For example, as clear from Figure 1 of Salim et al. (2007), the majority of galaxies in that study have $-24 \geqslant M_{r} \geqslant-18$, whereas nearly all of the ALFALFA dwarfs are less luminous than $M_{r}$ "' -18 , with the mean $M_{r} \sim-15$. Second, $\mathrm{Hi}$ selection results in no obvious color bimodality in dwarfs, because nearly all $\mathrm{H}$ i-bearing galaxies are blue, actively star-forming systems; $\mathrm{H}$ i selection is highly biased against the red sequence (West et al. 2009; Haynes et al. 2011). Based on the distribution of their full GALEX-SDSS-matched catalog, Salim et al. (2007) identified blue-cloud galaxies as those with $m_{\mathrm{NUV}}-r \diamond 4$. Given the color-magnitude relation, fainter galaxies are bluer on average and the dividing criterion shifts to $m_{\mathrm{NUV}}-r \sim 3.5$ at $M_{r} \sim-17$, according to Figure 1 in Kim et al. (2010). As evident in Figure 9, virtually all the ALFALFA dwarf galaxies are below the division of $m_{\mathrm{NUV}}-r=4$. Only three objects, UGC 6245, AGC 223913 = VCC 1649, and AGC $222297=$ VCC 180 (the latter two early type galaxies associated with the Virgo Cluster), have $m_{\mathrm{NUV}}-r>3.5$, lying in the green valley or blue edge of the red sequence. At fixed $M_{r}$, galaxies with lower $M_{\mathrm{H} \text { i }}$ (the filled circles) have, on average, redder colors, indicating that they also exhibit a lower gas fraction $\left(\boldsymbol{f}_{\text {gas }}\right.$, defined as $M_{\mathrm{H} \text { i }} / M_{*}$ throughout this work); this trend confirms the general association of lower $\mathcal{f}_{\text {gas }}$ and redder color (see also Section 5.3). As a result, in the range of $-18 \vartheta M_{r}>-10$, a population of extremely low gas fraction $\mathrm{dE} / \mathrm{dSph}$ would have $M_{\mathrm{H} \text { i }}$ lower than the detection limit of ALFALFA; they would sit on the red sequence in this low-luminosity range but are not included in this sample. This point is further discussed in the case of the Virgo Cluster by G. Hallenbeck et al. (2012, submitted). In addition, there is a trend for lower luminosity objects to have bluer colors, as is also found in studies of large samples of massive galaxies (Wyder et al. 2007). As is the case with the full ALFALFA sample (Haynes et al. 2011; S. Huang et al. 2012, in preparation), the CMD shown in Figure 9 confirms that, in comparison with large optical-UV samples, an $\mathrm{H}$ i-selected dwarf sample is highly biased against red sequence galaxies.

The right panel of Figure 9 presents a color-color plot
with the horizontal axis showing the optical color $(u-r)$. Not surprisingly there is a strong correlation such that as a galaxy becomes redder in $m_{\mathrm{NUV}}-r$, it also becomes redder in $u-r$. As found by Wyder et al. (2007) for blue galaxies, the two colors are very well correlated with a slope of $\delta(u-r) / \delta\left(m_{\mathrm{NUV}}-r\right) \sim 0.5$; this slope is roughly consistent for the ALFALFA dwarf sample. Those authors also found that, for galaxies with colors redder than $m_{\mathrm{NUV}}-r \approx 3.5$, the $u-r$ color begins to increase less quickly with $m_{\mathrm{NUV}}-r$ than for bluer $u-r$ colors. The ALFALFA sample shows no obvious change in slope, as expected since most lie on the blue side of the division.

We also examine the $m_{\mathrm{FUV}}-m_{\mathrm{NUV}}$ color, a sensitive probe of the rate of current SF. There are 117 galaxies (69 belonging to the complete $s$-com sample and 48 to the supplementary s-sup one) that have detectable magnitudes in both the FUV and the NUV. As described in Gil de Paz et al. (2007), late-type spiral and irregular "blue-cloud" galaxies can be roughly separated

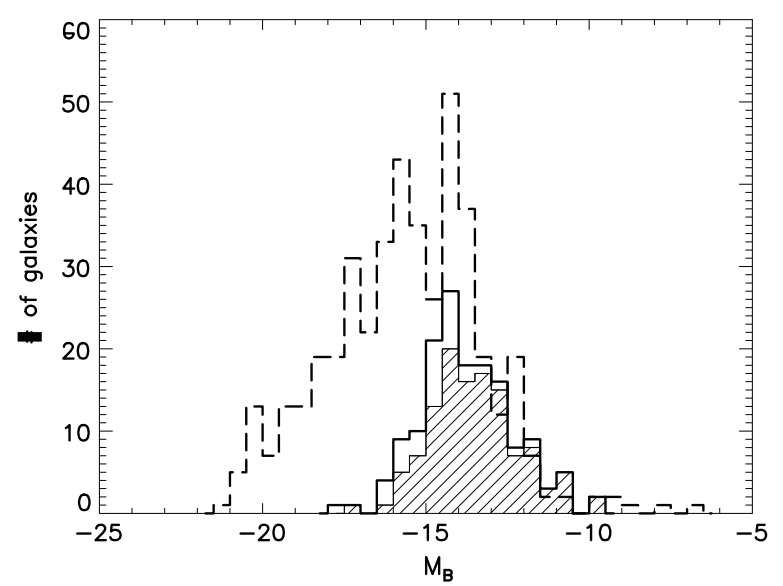

Figure 10. $B$-band absolute magnitude distribution of the 152 galaxies with acceptable SDSS magnitudes. Solid histograms indicate the ALFALFA dwarf galaxies, with the shaded area tracing the $s$-com sample and the open area the s-sup galaxies. In comparison, the dashed histogram shows the distribution for the 11HUGS sample. Although the s-com sample extends to lower $\mathrm{H}$ i mass and its galaxies have, on average, lower gas fractions, both ALFALFA and 11HUGS probe to comparably faint optical absolute magnitudes.

from early-type "red-sequence" galaxies using a division at $m_{\mathrm{FUV}}-m_{\mathrm{NUV}}=0.9$. According to that demarcation, $92 \%$ of our galaxies fall on the blue side. $72 \%$ of the combined ( $s$-com plus $s$-sup) sample have $m_{\mathrm{FUV}}-m_{\mathrm{NUV}}<0.5$; among the lower $\mathrm{H}$ i mass $s$-com galaxies, this percentage drops to $64 \%$. Furthermore, the median UV color is $0.36 \mathrm{mag}$ for the $s$-com plus s-sup galaxies ( $0.42 \mathrm{mag}$ for the $s$-com and $0.31 \mathrm{mag}$ for the $s$-sup galaxies). Evaluation of the K-S statistic shows that the probability that the UV color distributions of the two samples ( $s$-com versus $s$-sup) are drawn from the same underlying distribution is only $2.6 \%$. The galaxies with the lowest $\mathrm{H}$ i mass $(s-\mathrm{com})$ are redder than comparable galaxies with higher $\mathrm{H} \mathrm{i}$ masses, that is, the $\mathrm{H}$ i mass cutoff imposed on the $s$-com results in a sample with redder UV colors on average at fixed optical luminosity (see also Section 5.3).

In comparison, the sample studied by Lee et al. (2011), mainly drawn from the $11 \mathrm{HUGS}$, has $79 \%$ of the galaxies with $m_{\mathrm{FUV}}-m_{\mathrm{NUV}}<0.5$ and a median UV color of 0.29 mag, bluer than the ALFALFA dwarf galaxies, especially the $s$-com ones. Figure 10 examines more closely the distribution of absolute magnitude $M_{B}$ for the ALFALFA dwarf and 11HUGS samples. The solid histograms trace the distribution of the 152 ALFALFA dwarfs with acceptable SDSS magnitudes (see Section 3.3), with the shaded area identifying the complete $s$-com sample and the open area the $s$-sup galaxies. To convert the SDSS bands to $B$ band, we use the transformation, $B=g+0.3130(g-r)+0.2271$. For comparison, the dashed histogram represents the parent 11 HUGS sample with their $B$-band absolute magnitudes drawn from Table 3 of Kennicutt et al. (2008). Although the ALFALFA $s$-com sample extends to considerably lower $\mathrm{Hi}$ mass, the faintest optical magnitudes probed by both ALFALFA and 11HUGS are similar. Relative to the 11HUGS galaxies, the lower gas fractions typical of the $s$-com dwarfs are consistent with their redder colors.

One of the ALFALFA dwarfs, AGC $223913=$ VCC 1649 (see its images in Figure 7 ), even has $m_{\mathrm{FUV}}-m_{\mathrm{NUV}}=3$, implying that it is in a quiescent stage of SF. It is a dE/dSph in Virgo, whose red UV color could result from a recent quenching of SF activity, possibly due to gas loss by ram pressure stripping (e.g., Boselli et al. 2008). This object and the other 
early-type dwarfs detected by ALFALFA in the Virgo Cluster are discussed in more detail elsewhere (G. Hallenbeck et al. 2012, in preparation).

\section{THE DERIVATION OF THE PHYSICAL PROPERTIES OF ALFALFA DWARF GALAXIES}

In this section, we use the data sets discussed above to derive the physical properties of the ALFALFA dwarf galaxies $(s$-sed $)$ : their stellar masses, internal extinction, metallicity, and SFR.

\subsection{SED Fitting}

While studies of resolved stellar populations provide the best means to determine the SFHs of galaxies, it is not yet feasible to conduct such studies on the majority of the ALFALFA dwarfs. Hence, we analyze their integrated light and global properties by SED fitting, following the method of Salim et al. (2007), with a slight change in the prior distribution of the effective optical depth in the $V$ band, $\tau_{V}$ (see Section 4.3). Of the full ALFALFA dwarf sample, reprocessed FUV and NUV magnitudes and acceptable-quality SDSS pipeline photometry as described above are available for a total of 74 galaxies, 45 belonging to the more restrictive s-com sample and 29 to the additional s-sup set. The full likelihood distributions of parameters are derived for this combined sample, referred to hereafter as $s$-sed, by fitting the seven observed SDSS (ugriz) and GALEX (FUV NUV) bands to an extensive library of model SEDs (Gallazzi et al. 2005), generated using the B\&C 03 stellar population synthesis code (Bruzual \& Charlot 2003). Dust is accounted for with the Charlot \& Fall (2000) two-component model to include attenuation from both the diffuse interstellar medium (ISM) and short-lived (10 Myr) giant molecular clouds. It is proposed to produce consistent treatment for both the $\mathrm{H} \alpha$ and UV continuum attenuation. However, we do note that recent studies show that the extinction curve may be a function of stellar mass, SSFR, axis ratio, stellar surface mass density, etc. (e.g., Johnson et al. 2007; Wild et al. 2011). A Chabrier (2003) IMF is assumed, and random bursts are allowed to be superimposed on a continuous $\mathrm{SFH}$.

A concern for the study of $\mathrm{Hi}$-selected galaxies is whether the parameter space used for building the library is set wide enough to cover the intrinsic values of the metal- and dustpoor ALFALFA dwarfs. For example, following Salim et al. (2007), the effective $V$-band optical depth lies in the range $0:\left(\tau_{V}<6\right.$, and the $\mu$ factor, i.e., the fraction of the optical depth that affects stellar populations older than $10 \mathrm{Myr}$, varies from 0.1 to 1 . A commonly used prescription by Calzetti (1997) adopts $E(B-V)_{\text {star }}=0.44 E(B-V)_{\text {line }}$ (see also discussion in Section 4.3). Furthermore, the metallicity of the stellar population is uniformly distributed between 0.1 and $2 Z_{0}$. In particular, we can anticipate that in some cases, the emission lines may be so strong that the observed colors may deviate from the adopted line-free continuum model (Salim et al. 2007; West et al. 2009), e.g., the $\mathrm{H} \alpha$ emission may dominate the $r$-band luminosity to offset the observed $r-i$ color blueward of the models. However, outliers affected by line emission can be identified in a color-color plot, e.g., $r-i$ versus $g-r$. As a

result, the $\chi^{2}$ value of the best-fitting model, $\chi_{\text {best }}^{2}$, would have a mean larger than that predicted by the degree of freedom of the fitting. We checked the distribution of $\chi_{\text {best }}^{2}$ and found that it is generally good, implying that the library does, in fact, reproduce most of the observed SEDs. However, there is a tail of large $\chi_{\text {best }}^{2}$ objects, and we confirm by visual inspection that their deviation is likely caused by strong line emission (e.g., AGC $220856=$ VCC 1744 and AGC $223390=$ VCC 274, two Virgo BCDs; the SDSS and GALEX images of the former are included in Figure 7). Additionally, although we already excluded the "shredded" sources, there remain a few objects with suspicious SDSS magnitudes, e.g., AGC 205165 (problematic de-blending of a superposed star) and AGC 191791 = LSBC D634-03 (unusual color in the SDSS image), which also lead to large $\chi_{\text {best }}^{2}$. For these reasons, we emphasize that the SED fitting results should be interpreted only in a statistical sense, with these large $\chi_{\text {best }}^{2}$ objects being less reliable. The galaxies with $\chi_{\text {best }}^{2}<10$ are noted by a "*" over the AGC numbers in Table 1.

Compared to SED fitting to the optical SDSS bands only, fitting with the addition of the GALEX UV bands yields better constraints on the parameters, especially on the dust optical depth and the SFR over a timescale of $100 \mathrm{Myr}$, comparable to the lifetime of UV-bright stars (Salim et al. 2005). We use the likelihood-weighted average as our nominal estimate of the logarithm of the parameter value, rather than the mode, to avoid sensitivity to binning, and $1 / 4$ of the $2.5-97.5$ percentile range as a proxy for what would have been the uncertainty estimate in the Gaussian distribution (Kauffmann et al. 2003b; Salim et al. 2007). An extra term that accounts for distance uncertainties is added for distance-dependent quantities, e.g., the stellar mass and SFR. This term always dominates the SED fitting error (characterized by the probability distribution function, PDF) for the nearby dwarfs in the stellar mass estimates, but not for the errors in the SFRs. Note that systematic uncertainty is not included in the error estimate. When the UV bands are excluded, the median uncertainty in the $\log M_{*}$ estimate is 0.217 ; in $\log \tau_{V}$, it is 0.455 ; in $\log$ SFR, it is 0.323 . When the UV data are incorporated, those values decrease to $0.212,0.367$, and 0.277 , corresponding to median improvement of $1 \%, 21 \%$, and $7 \%$, respectively. While the constraint on the stellar mass is least improved, the incorporation of the UV data is critical to the other parameters, both of which are more sensitive to changes in the UV luminosity.

\subsection{Stellar Mass}

Based on the SED fitting error term characterized by the PDF, the $M_{*}$ is the best constrained parameter in the SED fitting. The logarithm of $M_{*}$, in units of solar mass, is listed in Column (11) of Table $1 .\left(\log M_{*}\right)=7.40$ for the $s$-sed dwarfs $(7.28$ for the lower $\mathrm{Hi}$ mass $s$-com galaxies and 7.58 for the additional s-sup objects), significantly lower than those of typical SDSS or GALEX samples (Brinchmann et al. 2004; Salim et al. 2007). This result further confirms that the imposition of an $\mathrm{Hi}$ line width cutoff effectively eliminates most of the $\mathrm{Hi}$ deficient but high-luminosity objects. The only two galaxies with $\log M_{*}>8.5$ are UGC 6245, a low-luminosity SB0 galaxy viewed almost face-on so that its true velocity width may be much larger, and UGC 7889 (=NGC 4641), a BCD in Virgo. The former has the highest stellar mass $\log M_{*}=9.52$ and a low gas fraction but is left in the s-sup sample because of the availability of its GALEX image from our GI program (see Section 2).

Another widely used method to estimate the stellar mass from optical magnitudes employs the relation of mass-to-light ratio versus color as calibrated by Bell et al. (2003). However, the SFH adopted by Bell et al. (2003) does not fully account for the impact of the bursty behavior seen in nearby dwarfs (Lee et al. 2009b). Gas-rich dwarfs have $b$-parameters ( $b \equiv$ SFR/SFR), the current SFRs normalized to the average past SFRs) that 
are high on average (see Section 5.1). As a result, application of the standard Bell et al. (2003) calibration would produce stellar masses that are more massive for the same optical color. Additionally, at low redshift, $\mathrm{H} \alpha$ emission may contribute to the $r$-band flux and [O iii] and $\mathrm{H} \beta$ to that at the $g$ band. On the other hand, the $g-r$ color is largely unchanged (West et al. 2009) and the $i$ band contains almost no emission lines. Therefore, following West et al. (2009), we also calculate the stellar mass using the $i$-band luminosity and the $g-r$ color. We apply $K$-corrections using the IDL code "kcorrect" ( $\left.v 4 \_14\right)$, described in Blanton \& Roweis (2007), and estimate internal extinction corrections based on Equation (12) in Giovanelli et al. (1997). The latter has little effect on the stellar mass estimate, since when the luminosity is increased, the color also becomes bluer by an associated amount such that the two effects cancel each other (Bell et al. 2003).

The two stellar mass estimates, i.e., that derived from Bell et al. (2003) calibration versus that derived from SED fitting, agree for the most massive galaxies with $M_{*} \curvearrowright 10^{10} M_{0}$ and low $b$-parameters, but systematic deviations between the two become non-negligible at the low-mass end. While the Bell et al. (2003) method gives a median $\log M_{*}=7.73$ for the $s$-sed ALFALFA dwarfs (converted to Chabrier IMF), SED fitting yields a considerably lower median of 7.45 corresponding to a factor of two difference. Furthermore, because the most gas-rich galaxies (i.e., higher $M_{\mathrm{H}}$ for their $M_{*}$ ) with higher $b$-parameters in this stellar mass range are excluded from the dwarf sample in this work (see Section 5), the deviation is even larger among the full low stellar mass ALFALFA population (S. Huang et.al. 2012, in preparation), We find a discrepancy of similar magnitude to that found by Wyder et al. (2007), 0.4 dex

on average at $10^{8} M_{U}$, and we confirm that the galaxies that are estimated to be more massive by the Bell et al. (2003) method have generally higher $b$-parameters. To incorporate reasonably the effects of stochastic SF, bursty behavior in dwarf galaxies should be a key feature of modeling the SEDs.

\subsection{Internal Extinction}

Although the FUV luminosity provides a more robust estimate of the recent SFR in dwarf galaxies than optical measures, it is subject to significant uncertainties because of the required dust attenuation corrections, which themselves are subject to large scatter produced by the large range of possible dust content, dust distribution, and geometry relative to the stars and ISM in galaxies. Even at $0.02 Z_{0}$, I Zw 18 contains a non-negligible amount of reddening, determined from optical observations to

be equivalent to $A_{V}=0.5$ (Cannon et al. 2002). In the dwarf galaxy SBS 0335-052 $\left(0.025 Z_{0}\right), A_{V}$ values as high as 20-30 have been suggested from MIR observations (Thuan et al. 1999). On the other hand, arguing that dwarf galaxies are expected to be extremely dust-poor, Roychowdhury et al. (2009) chose to neglect internal extinction entirely in their study of the FIGGS dwarf galaxy sample. Based on that assumption, they found a lower average $\Sigma_{\text {SFR }}$ for the FIGGS dwarfs than would be expected from the Kennicutt-Schmidt law (Kennicutt 1998b). Similarly, Hunter et al. (2010) took $E(B-V) \sim 0.05$ and Lee et al. (2009a) found $A_{\mathrm{H} \alpha}<0.1$ for the faintest galaxies. Here, rather than ignoring extinction, we have attempted to quantify its contribution by SED fitting using the optical/UV photometry.

Salim et al. (2007) already have pointed out the difference between the effective optical depth in the $V$ band derived from emission-line fitting, $\tau_{V, \mathrm{H} \alpha}$, and that derived from SED fitting, $\tau_{V, \mathrm{UV}}$, as a function of stellar mass. $\tau_{V, \mathrm{H} \alpha}$ arises to first or- der from the Balmer decrement, whereas the latter is mainly constrained by the UV spectral slope. At lower masses, $\tau_{V, \mathrm{UV}}$ is higher than $\tau_{V, \mathrm{H} \alpha}$, but the situation is reversed at the highmass end. According to those authors, $\tau_{V, \mathrm{UV}}-\tau_{V, \mathrm{H} \alpha} \sim 0.25 \mathrm{dex}$ (equivalent to $0.27 \mathrm{mag}$ in $A_{V}$ ) at $\log M_{*}=8.5$. This finding can be linked to that found by Wild et al. (2011) that $\tau_{V \text {, cont }} / \tau_{V \text {, line }}$ strongly increases with increasing SSFR, i.e., the galaxies with high SSFRs have a higher fraction of diffuse dust and their dust is more centrally concentrated. Adopting the same prior distribution of $\tau_{V}$ and $\mu$ as in Salim et al. (2007), we find, for the $s$-sed sample, mean values of $A_{\mathrm{FUV}}=1.47 \mathrm{mag}$ and $A_{\mathrm{NUV}}=1.08 \mathrm{mag}$; the Balmer decrement yields systematically smaller values of $A_{\mathrm{FUV}}$, assuming the Calzetti law (see below). In one object, UGC 7889 (=NGC 4641; see its images in Figure 7), the $A_{\mathrm{FUV}}$ value reaches 6.9 mag. This object is one of the few BCDs in Virgo detected by ALFALFA, and, among the dwarf $s$-sed sample, it is one of the reddest in $m_{\mathrm{FUV}}-m_{\mathrm{NUV}}$ color, has one of the highest stellar masses, has a low gas fraction, and is relatively metal-rich. Given its UV color, the high $A_{\text {FUV }}$ value of UGC 7889 derived by the SED fitting is consistent with that predicted by the IRX- $\beta$ relation (see below) as calibrated for starburst galaxies (Meurer et al. 1999), but other methods such as the Balmer decrement and the IRX-SFH-color relation (see below) give quite lower extinction estimates. Given the possible degeneracy of its red color arising from either high extinction or strong SF quenching in the Virgo environment, it is probable that the large extinction implied by the SED fitting for this galaxy is unreliable.

Another approach that we suggest is relevant here anticipates that extinction should be lower in less luminous and face-on systems with low metallicities (e.g., Giovanelli et al. 1995; Xiao et al. 2012). Salim et al. (2007) showed that the derived $\tau_{V, \mathrm{UV}}$ is sensitive to the assumed prior distribution of $\tau_{V}$ in the model library. Therefore, to constrain better $\tau_{V \text {,UV }}$, we first try to estimate $\tau_{V}$, following Giovanelli et al. (1995), and trim out the models with unrealistically high extinctions. With this improvement in the SED fitting, the mean values for the ALFALFA dwarf $s$-sed sample become $A_{\mathrm{FUV}}=1.33 \mathrm{mag}$ and $A_{\mathrm{NUV}}=0.96 \mathrm{mag}$, with formal uncertainties of 0.42 and $0.32 \mathrm{mag}$, respectively. In particular, the $A_{\mathrm{FUV}}$ value is reduced to $2.36 \mathrm{mag}$ for UGC 7889. Since the $A_{\mathrm{FUV}}$ estimates are reduced by 0.14 mag on average, the log SFR is reduced correspondingly by 0.06 dex (see values in Section 4.5). As noted previously (Section 4.2), such improvement has little effect on estimates of stellar mass.

To convert the extinction to an estimate of the dust mass, we adopted Equation (44) from Popescu et al. (2011), $M_{\text {dust }}=$ $0.9912\left[M_{0} \mathrm{pc}^{-2}\right] h_{S}^{2} \tau_{B}^{f}$, where $h_{S}$ is the stellar disk scale length in parsec and $\tau_{B}^{f}$ is the face-on optical depth in the $B$ band. SDSS $g$-band values are used to approximate values in the $B$ band. Following Giovanelli et al. (1994) and Shao et al. (2007), the observed disk scale lengths and optical depths are converted to face-on values. The resulting dust-to- $\mathrm{H} \mathrm{i}$ gas mass ratio is 0.003 on average for the $s$-sed galaxies $(0.002$ for the $s$-sup sample and 0.004 for the lower $\mathrm{Hi}$ mass $s$-com population); this ratio is below the mean value of 0.007 quoted in Draine et al. (2007). In comparison, a dust-to-gas ratio of 0.002 was determined in the nearby starbursting dwarf galaxy NGC 4214 (Lisenfeld et al. 2011). We also note that Boissier et al. (2004) found that the dust-to-gas ratio is proportional to $\left(Z / Z_{0}\right)^{0.88}$.

In order to assess fairly the accuracy of our results, we have examined correlations between the FUV extinction $A_{\mathrm{FUV}}$ and various relevant quantities, in particular those for which relations have been established for samples of more massive 
galaxies. We find no significant trend with axial ratio, mainly because our sample includes no high axial ratio systems; nearly all show $\log a / b<0.5$. As discussed in Section 2.4, it is unlikely that we are missing edge-on dwarfs because of the line width cutoff. Rather, the observed distribution implies that dwarf galaxies are intrinsically thicker or that the distribution of SF sites within them is quite irregular, making the tracing of their disks more complicated. Similarly, Xiao et al. (2012) suggested that dust reddening is not so sensitive to axial ratio at low metallicity as at high metallicity. We have also looked for trends in $A_{\mathrm{FUV}}$ with $M_{*}$ and $\mathcal{f}_{\text {gas }}$. The trend seen by Salim et al. (2005) that attenuation increases with stellar mass is not evident among the ALFALFA dwarf galaxies; the latter, of course, include no really massive galaxies. However, in comparison with Figure 2 of Salim et al. (2005), we find that the highest value of $A_{\mathrm{FUV}}$ reached by gas-rich galaxies (as identified by high $f_{\text {gas }}$ ) is lower than that estimated for relatively gas-poor galaxies. Such a result is reasonable assuming that the galaxies that are gas-rich for their stellar masses are less evolved, have lower dust content and metallicity, and thus exhibit less attenuation.

Other common approaches to estimating extinction exploit the infrared wavelengths. In this case, however, the Spitzer archive contains too few of the ALFALFA dwarfs to permit a direct measurement of the infrared excess (IRX) incorporating MIPS wavelengths. Instead, we examine how $A_{\mathrm{FUV}}$ varies with UV color $(\beta)$, equivalent to the IRX- $\beta$ relation following Salim et al. (2007). In comparison with the relation given for normal star-forming galaxies by those authors, the ALFALFA dwarfs only marginally follow the trend, with the more massive $s$-sup members showing less scatter about the relation. A number of the lowest $\mathrm{H}$ i mass $s$-com galaxies fall below the relation for normal galaxy samples; their $A_{\mathrm{FUV}}$ would thus be overpredicted by the standard IRX $-\beta$ relation given their UV color, possibly because of their different SFH and/or dust geometry (Kong et al. 2004; Cortese et al. 2006). We also find that $A_{\mathrm{FUV}}$ is better correlated with the $m_{\mathrm{FUV}}-m_{\mathrm{NUV}}$ color than with the $m_{\mathrm{NUV}}-r$ color.

In addition, we use the spectroscopic Balmer lines to compare the observed flux ratios in the $\mathrm{H} \alpha$ to $\mathrm{H} \beta$ lines with the theoretical value (2.86 for Case $\mathrm{B}$ recombination) to estimate $A_{\mathrm{H} \alpha}$. We assume that $A_{\mathrm{FUV}}=1.8 A_{\mathrm{H} \alpha}$, following the Calzetti law (Calzetti et al. 2000), with the caveats about large variations in UV extinction curves and aperture effects. We approximate the ratios using the line flux measurements from the MPAJHU DR7 catalog (see Section 3.3). This method is applied to 42 galaxies that are common to both the s-sed sample and the MPA-JHU catalog. Surprisingly, this crude application of the Balmer decrement method gives systematically smaller extinction estimates, with a mean $A_{\mathrm{FUV}}=0.64 \mathrm{mag}$ when we adopt the Calzetti law. Given that the $A_{\mathrm{FUV}}$ values are presumably dominated by attenuation by the diffuse ISM whereas the emission lines probe the local attenuation within the clouds in which they originate, one might expect the opposite result as is found in massive galaxies (Calzetti et al. 2000) and incorporated into the factor of 1.8 between the FUV and $\mathrm{H} \alpha$ extinctions. However, we note that Wild et al. (2011) also found a strong decrease in emission line-to-continuum attenuation ratio with increasing SSFR, in agreement with the overall high SSFRs of the ALFALFA dwarf sample relative to the massive galaxies (see Section 5.1) and likely indicative of the variation of the dust geometry with the SSFR. Furthermore, there is large uncertainty in the UV extinction curve so that the constant in the $A_{\mathrm{FUV}}=1.8 A_{\mathrm{H} \alpha}$ relation suffers from large uncertainty and is most likely underestimated. For the low stellar surface mass density galaxies $\left(\mu_{*}<3 \times 10^{8} M_{\cup} \mathrm{kpc}^{-2}\right)$ with $A_{V}=1$, Wild et al. (2011) found $0.3 \mathrm{mag}$ more attenuation in the NUV compared to the Calzetti law that is derived for starburst galaxies. Similarly, Buat et al. (2011) found a steeper dust extinction curve in ordinary star-forming galaxies than found in starburst galaxies by Calzetti et al. (2000). In addition, the bump strength of the $2175 \AA$ dust feature is known to vary. Incorrectly assuming a dust curve with no $2175 \AA$ feature (e.g., the Calzetti law for starburst galaxies) would lead to an underestimate in the amount of dust, in turn leading to the incorrect conclusion that the stellar populations are extremely metal-poor (Wild et al. 2011). In particular, the steep FUV rise and small $2175 \AA$ feature in dwarf galaxies, resembling the observed extinction curve of some regions in the LMC, may be due to different dust grain properties in low-mass galaxies (e.g., Galliano et al. 2003).

As a final approach, we have explored the extended IRX-SFH-color relation based on the $m_{\mathrm{NUV}}-r$ color and the $D_{n}$ (4000) measurement derived in Johnson et al. (2006), which is commonly used by local GALEX studies (Wyder et al. 2007; Schiminovich et al. 2007); application of this relation to the ALFALFA dwarfs predicts a much larger extinction $A_{\mathrm{FUV}}$ in many of the lowest mass $s$-com galaxies.

At the end of this analysis of the various methods of estimating the internal extinction, we conclude that (1) SED fitting using the UV continuum is the most reliable currently available tool to obtain $A_{\mathrm{FUV}}$ values for the ALFALFA dwarfs, but that (2) there is large uncertainty in the extinction estimates. It is inappropriate to ignore completely a correction for extinction at UV wavelengths (the mean of $A_{\mathrm{FUV}}$ is $1.33 \mathrm{mag}$ ), although attenuation in the dwarfs is smaller than that found in more massive SDSS galaxies, e.g., $2.03 \mathrm{mag}$ in Salim et al. (2005). We note that taking attenuation into account can bring the dwarf galaxies in the FIGGS study of Roychowdhury et al. (2009) closer to the Kennicutt-Schmidt SF law obtained for more massive galaxies. However, it will not lead to a change in the slope of the relation. As was found by Roychowdhury et al. (2009), the relationship between the $\mathrm{H}$ i density and the SFR in gas-rich dwarfs is steeper than the Kennicutt-Schmidt SF law.

\subsection{Metallicity}

Standard recipes used to convert FUV luminosities to SFRs (e.g., Kennicutt 1998a) are derived on the assumption of solar metallicity stellar populations, $Z_{\mathrm{O}}$. However, in the general population of star-forming galaxies, the gas-phase metallicity $Z$ of the ISM is known to increase with stellar mass, e.g., Tremonti et al. (2004). This dependence is consistent with simple closed-box models (e.g., Garnett 2002), whereby high gas fraction galaxies are relatively unevolved and thus have experienced low metal enrichment. Especially in low-mass systems, ISM blowout can follow episodic SF events. The adoption of an incorrect $Z$ to estimate the SFR through the observed FUV luminosity can significantly bias the result, as a relative deficiency of metals would result in higher temperatures in the photospheres of forming stars and thus in a greater number of UV photons produced per unit stellar mass.

It is thus legitimate to question the validity of SFRs derived for low $M_{*}$ systems such as the ALFALFA dwarfs, if an account for their presumed low $Z$ is not considered. Short of specific measurements through optical spectroscopy, the stellar population metallicity may be estimated via SED fitting. Unlike other well-constrained parameters such as $M_{*}$, however, $Z$ is 
poorly constrained by SED fitting; in fact, deriving $Z$ from SED fitting often yields a likelihood distribution with multiple peaks. While we would normally expect that the mean estimates of metallicity $(Z)$ derived for larger samples are more reliable, we note that the Salim et al. (2005) sample yields $Z$ "' $Z_{\mathrm{O}}$, while for the ALFALFA dwarf s-sed sample we obtain $0.48 Z_{0}$ $\left(0.44 Z_{\mathrm{O}}\right.$ for the low $\mathrm{Hi}$ mass $s$-com galaxies and $0.54 Z_{\mathrm{O}}$ for the additional $s$-sup ones). Therefore, for our estimates of SFR, we adopt Kennicutt's relation, with a correction of 1.1 as given by Hunter et al. (2010), appropriate for a Salpeter IMF and $Z \sim 0.4 Z_{\mathrm{O}}$.

The stellar population metallicity is correlated with gas-phase metallicity, though with large scatter (Gallazzi et al. 2005). Given the shallow potential well of dwarf galaxies, the gasphase metal enrichment can be easily lost by outflow. Oxygen abundance measures from the MPA-JHU DR7 release of SDSS spectra (Tremonti et al. 2004) are available for 58 ALFALFA dwarf galaxies, with a mean of $12+\log (\mathrm{O} / \mathrm{H})=8.26(8.27$ for 44 low $\mathrm{Hi}$ mass $s$-com members and 8.22 for $14 \mathrm{~s}$-sup galaxies). The oxygen abundances can be converted to gasphase metallicities in units of solar metallicity $\left(Z_{0}=0.02\right)$, adopting a value of $12+\log (\mathrm{O} / \mathrm{H})_{\mathrm{O}}=8.66$ (Asplund et al. 2004). The mean of the gas-phase metallicity for the ALFALFA dwarf galaxies corresponds to $0.4 Z_{0}$. However, according to the mass-metallicity relation derived in Tremonti et al. (2004), based mainly on more massive galaxies $8.5<\log M_{*}<$ 11.5 , the stellar mass of $\log M_{*}=7.4$ corresponds to $12+$ $\log (\mathrm{O} / \mathrm{H})=7.78$. Almost all of the ALFALFA dwarfs lie above this relation.

\subsection{Star Formation Rates}

As a next step, we derive the global SFR for the ALFALFA dwarf galaxies both from the FUV luminosity alone, SFR FUV $_{\text {, }}$

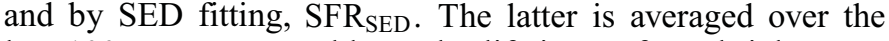
last $100 \mathrm{Myr}$, comparable to the lifetimes of UV-bright stars. Compared to SFRs derived from the $\mathrm{H} \alpha$ luminosity, both

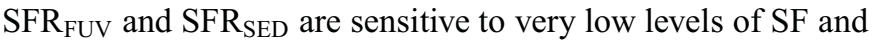
hence are particularly relevant for application to the ALFALFA dwarf sample.

SFR $_{\text {SED }}$ values are given, in logarithmic units of $M_{\cup} \mathrm{yr}^{-1}$, in Column (12) of Table 1 for the ALFALFA $s$-sed galaxies. The mean is - $2.18(-2.48$ for the lower Hi mass $s$-com sample and -1.71 for the $s$-sup galaxies), within a minimum of $\sim-4$. In comparison with values found for typical SDSS samples (Salim et al. 2005), the ALFALFA dwarfs overall show very low values of SFR. In such a regime where only a handful of $\mathrm{O}$ stars are formed over timescales comparable to their lifetimes (i.e., a few million years), $\mathrm{H} \alpha$ emission will appear weak or even absent. The SFRs inferred from $\mathrm{H} \alpha$ deviate from those of FUV at $\log$ SFR " ' -2 , but such stochastic effects may begin to play a role below log SFR " ' -3, according to Lee et al. (2009a). Lowmass stars may form continuously but at a very low rate so that no change in the IMF is required. However, Lee et al. (2009a) have shown that other factors, including internal dust attenuation, stellar model uncertainties, metallicity, ionizing photon loss, and starbursts, if considered alone, are insufficient to explain the amplitude of the underestimate of the SFR derived from $\mathrm{H} \alpha$ relative to that implied by the UV luminosity. They also suggested that an IMF deficient in high-mass stars is consistent with their results. While the current data set is insufficient to provide conclusive insight, the ALFALFA dwarfs with their very low SFRs will provide an ideal opportunity to test the hypothesis of a non-universal IMF.
To obtain $\mathrm{SFR}_{\mathrm{FUV}}$, a commonly used standard conversion factor from the dust-corrected FUV luminosity into the SFR is given by Kennicutt (1998a), assuming solar metallicity, a Salpeter IMF, and a constant SFH over at least the past $\sim 100 \mathrm{Myr}$ in the stellar mass interval of $0.1 M_{0}-100 M_{0}$ :

$$
\operatorname{SFR}\left[M_{0} \mathrm{yr}^{-1}\right]=1.4 \times 10^{-28} L_{v}\left[\mathrm{erg} \mathrm{s}^{-1} \mathrm{~Hz}^{-1}\right]
$$

where the FUV flux is deriyed from the $\mathrm{AB}$ magnitude (Lee et al. 2009a): $\mathcal{f}[\mathrm{mJy}]=10$. Taking into account the sub-solar metallicity (see Section 4.4), and adopting a Chabrier IMF that predicts a smaller SFR for the same luminosity than a Salpeter IMF by a factor of 1.58 (Salim et al. 2007), Equation (1) becomes

$$
\operatorname{SFR}\left[M_{v} \mathrm{yr}^{-1}\right]=0.81 \times 10^{-28} L_{v}\left[\mathrm{erg} \mathrm{s}^{-1} \mathrm{~Hz}^{-1}\right]
$$

The upper panels in Figure 11 show a comparison of the two

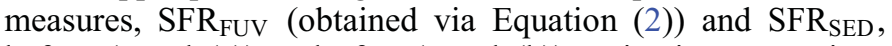
before (panel (a)) and after (panel (b)) extinction corrections are applied, for all the ALFALFA $s$-sed galaxies. Filled circles denote the low Hi mass $s$-com members, whereas open ones identify the additional s-sup galaxies. As evident in panel (a), in the absence of an extinction correction, the FUV luminosity underestimates the SFR, because, although small, the extinction at UV wavelengths is not negligible. Moreover, the deviation from the one-to-one relation (dashed line) is greater at the higher SFR end, suggesting that attenuation is larger for higher SFRs. Applying the extinction corrections $A_{\mathrm{FUV}}$ derived from the SED fitting (as discussed in Section 4.3) gives the result seen in panel (b): the scatter about and deviation from the one-to-one relation are significantly reduced. The relatively tight correlation evident here confirms that the stellar population synthesis model we used is consistent with what was used in Kennicutt (1998a).

However, as is still evident in panel (b), the FUV luminosity alone tends to overestimate the SFR compared to estimates derived from SED fitting, especially for the lowest SFRs, i.e., SFR below $\sim 10^{-2} M_{\cup} \mathrm{yr}^{-1}$. Although we do not compare the $\mathrm{SFR}_{\mathrm{FUV}}$ with $\mathrm{SFR}_{\mathrm{H} \alpha}$ here, we note that this threshold is close to the critical value identified by Lee et al. (2009a). Similarly, Salim et al. (2007) found that, since their sample is characterized by a wide variation in SFH, the conversion factor from FUV luminosity to SFR is smaller than that which would apply for a constant SFH. Compared to their sample, the ALFALFA dwarfs are currently forming more stars relative to the accumulated stellar masses (see Section 5.1). It is possible that the $b$ parameters become systematically larger in dwarf systems, i.e., a higher fraction of their stars have been formed more recently. For the same amount of observed FUV luminosity, the SFRs averaged over $100 \mathrm{Myr}$ would be smaller than those derived if the SFRs had remained constant. If so, the standard Kennicutt (1998a) conversion factor from $L_{\mathrm{FUV}}$ to SFR $\mathrm{FUV}$ would be too large in the case of dwarf galaxies. On the other hand, a bursty SF behavior would have less impact on the $\mathrm{SFR}_{\mathrm{H} \alpha}$, because the SFR is more likely to remain constant during the much shorter lifetimes of O-stars. These results suggest that the $\mathrm{SFR}_{\mathrm{H} \alpha}$ values not only may be suspicious (Lee et al. 2009a) but also

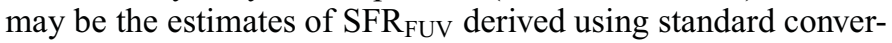
sions obtained for samples of more typical and more massive galaxies.

At this point, we do not have available $\mathrm{H} \alpha$ images, but we can make a crude attempt to quantify the SFRs with the $\mathrm{H} \alpha$ line flux derived from the SDSS spectra, $\mathrm{SFR}_{\mathrm{H} \alpha}$, for those galaxies with such measures. We again use the standard 
(a) Before attenuation correction

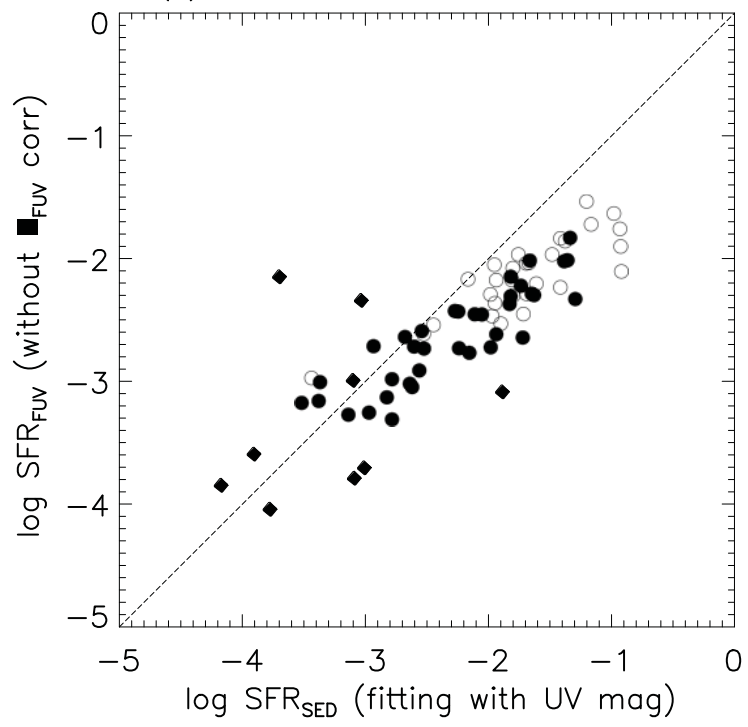

(c) Ha vs. FUV SFR

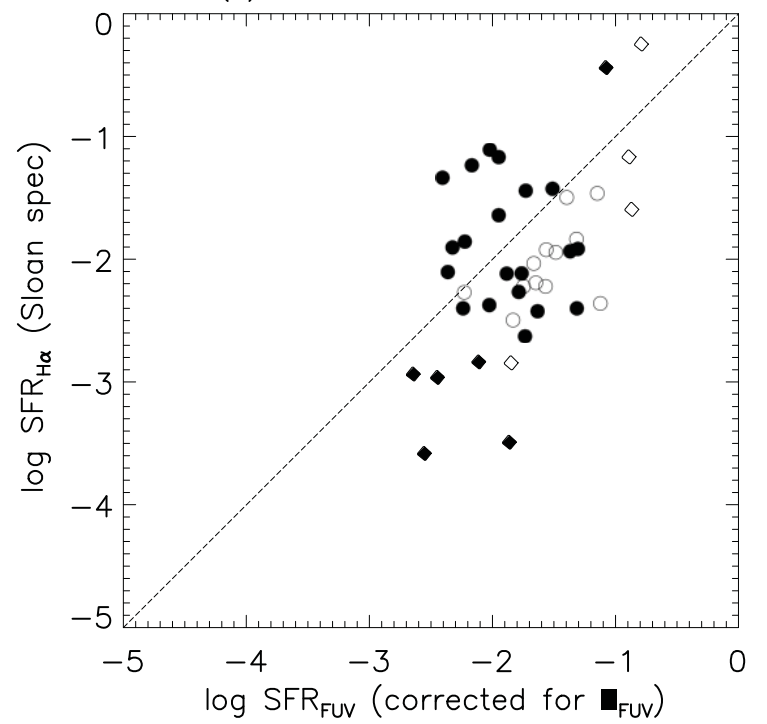

(b) Ifter attenuation correction

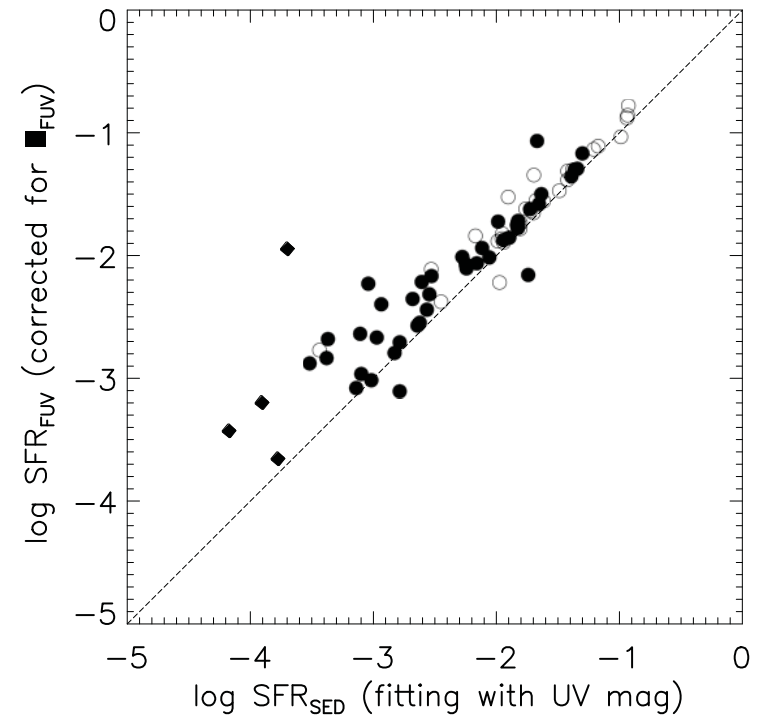

(d) Ha vs. SED SFR

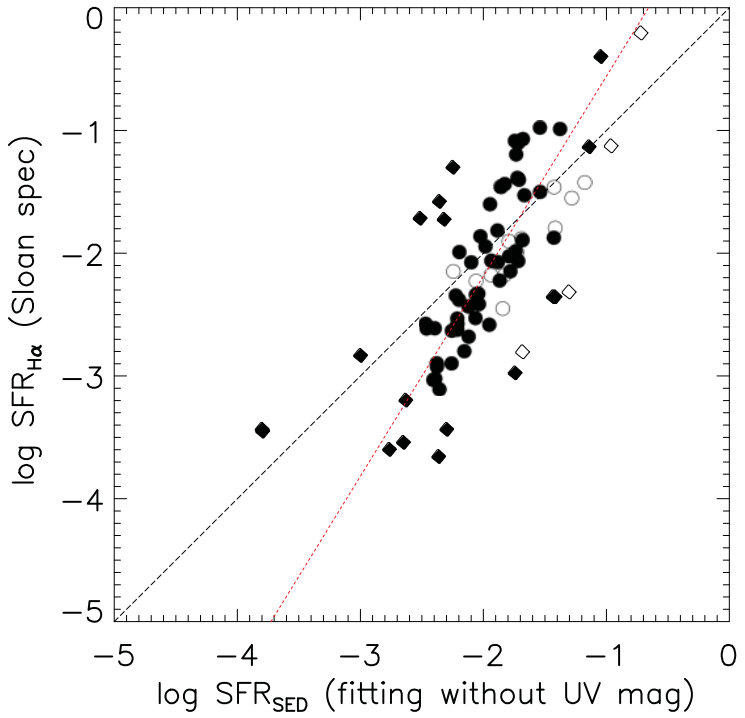

Figure 11. Comparison of SFR measures for the ALFALFA dwarf sample with open circles identifying the lower $\mathrm{H}$ i mass $s$-com galaxies and filled ones the additional s-sup galaxies in each panel. (a) SFRFUV obtained by the standard conversion from FUV luminosity, (Equation (2)) without attenuation correction, vs. SFR from SED fitting. (b) same as panel (a) except that $A_{\mathrm{FUV}}$, derived by SED fitting, have been applied to SFR FUV. The FUV magnitudes tend to overpredict SFRs below $\sim 10^{-2} M_{\cup} \mathrm{yr}^{-1}$, which may be due to the bursty nature of star formation in dwarfs. (c) SFR $\mathrm{FUV}$ compared with SFR $\mathrm{H} \alpha$, derived from the SDSS spectroscopic data (MPA-JHU DR7 measurements). Though our aperture correction tends to overestimate $\mathrm{SFR}_{\mathrm{H} \alpha}$, the deficiency of SFR $\mathrm{H}_{\alpha}$ is still visible. We are limited by the small number of the s-sed galaxies with usable SDSS data. (d) SFRs derived from SED fitting only to the SDSS bands compared with SFR $\mathrm{H}_{\alpha}$. A weak trend exists that H $\alpha$ underpredicts the SFR below $\sim 10^{-2} M_{\cup} \mathrm{yr}^{-1}$. The dash-dotted line is the linear fit to the data points, with a slope steeper than the one-to-one dashed line.

(A color version of this figure is available in the online journal.)

conversion factor calibrated in Kennicutt (1998a), taking into account the metallicity and IMF difference. The MPA-JHU reprocessed $\mathrm{H} \alpha$ line flux is corrected for attenuation, given by the Balmer decrement as in Section 4.3. We correct for the aperture effect by using the ratio of the fluxes corresponding to the entire galaxy $r$-band magnitude and the magnitude through the fiber in the same band, following Hopkins et al. (2003). The aperture correction implicitly assumes that the emission measured through the fiber is characteristic of the galaxy as a whole. However, in almost all of the ALFALFA dwarfs the $u-r$ colors are bluer within the fiber than across the galaxy, a fact that can be explained if the bluest starforming peaks in surface brightness are usually the targets of the spectroscopic observations. As a result, our crude scaling by the $r$-band light should overestimate $\mathrm{SFR}_{\mathrm{H} \alpha}$. However, the bottom panels in Figure 11 still show a systematic deficiency in $\mathrm{SFR}_{\mathrm{H} \alpha}$, relative to both $\mathrm{SFR}_{\mathrm{FUV}}$ (panel (c)) and $\mathrm{SFR}_{\mathrm{SED}}$ (panel (d)), albeit with large scatter. As evident in panel (c), the number of galaxies in the s-sed sample and with SDSS spectra is quite limited. In panel (d), we attempt to improve the statistics by dropping the requirement of UV data and thus calculating an SFR $_{\text {SED }}$ by fitting only to the SDSS bands. Albeit crude, the best linear fit line to the data points (the red dashed-dotted line) has a slope larger than 1, suggesting that while the $\mathrm{SFR}_{\mathrm{FUV}}$ could be an overestimate due to stochastic effects, we cannot rule out the possible systematic deficiency of $\mathrm{SFR}_{\mathrm{H} \alpha}$, compared to $\mathrm{SFR}_{\mathrm{SED}}$, and thus a non-universal IMF. We are in the process of obtaining $\mathrm{H} \alpha$ images for this sample and plan to present a more comprehensive comparison of SFR measures in a future work. 


\section{STARS, STAR FORMATION, AND GAS IN THE ALFALFA DWARFS}

Numerous previous works have explored the relationships between stars, SF, and gas in galaxies, and in this section, we explore how the ALFALFA dwarf galaxies, specifically the s-sed sample galaxies, compare to others. In comparison with results obtained for large samples derived from the SDSS (e.g., Brinchmann et al. 2004), the imposition of criteria associated with $\mathrm{Hi}$ selection and narrow $\mathrm{Hi}$ line width renders the ALFALFA dwarf sample a still diverse population of lowluminosity blue-cloud dwellers. In the context of examining the s-sed sample, it is therefore useful to compare it to a sample of higher mass objects selected by similar $\mathrm{H} \mathrm{i}$ criteria. To do that, we make use of a subset of 12,154 galaxies contained in the $\alpha .40$ catalog presented in Haynes et al. (2011) and which are also contained in the SDSS DR7. Haynes et al. (2011) present a cross-reference between the $\alpha .40$ and SDSS DR7 databases that is used as the basis for the parent $\mathrm{H} \mathrm{i}$-selected population. To derive stellar masses and SFRs for the larger sample, we make use here of the methodologies described in Section 4, but restricted only to the optical SDSS DR7 photometry. A more complete discussion of the $\alpha .40$-SDSS-GALEX sample will be presented elsewhere (S. Huang et al. 2012, in preparation).

\subsection{The ALFALFA Dwarfs on the Star-forming Sequence}

Besides the well-known correlation that the SFR increases with stellar mass (e.g., Brinchmann et al. 2004; Bothwell et al. 2009), the "star-forming sequence" in a specific SFR $\left(\mathrm{SSFR}=\mathrm{SFR} / M_{*}\right)$ versus stellar mass diagram is identified by the fact that the SSFRs of lower mass galaxies appear to be confined to a relatively narrow range of SSFR that declines as the stellar mass increases (Salim et al. 2007; Schiminovich et al. 2007). At masses above $\sim 10^{10} M_{\mathbf{U}}$, some galaxies exhibit much lower values of the SSFR, i.e., the sequence turns over. The tightness of the "star-forming" sequence strongly suggests that a galaxy's mass regulates its overall SFH. However, a second transition, near $M_{B} \sim-15$, has been reported (Lee et al. 2009b; Bothwell et al. 2009), below which the dispersion in SSFRs broadens again: low-luminosity, low-mass galaxies also show a wide range of SSFR. Based on a sample of 1000 galaxies with both $\mathrm{H} \alpha$ and $\mathrm{Hi}$ measures available in the literature, Bothwell

et al. (2009) have argued that possible causes of this behavior can be categorized under two main headings; first, the various physical mechanisms that underlie the SF properties of dwarf galaxies not only exhibit a large spread but also are decoupled from their gas contents. A second possibility proposed by those authors is that at low luminosities, the SFR is not faithfully traced by the $\mathrm{H} \alpha$ luminosity, leading to a mischaracterization of the SFR. Since the SSFRs used here are based on SED fitting, the second explanation proposed in Bothwell et al. (2009), i.e., that $\mathrm{H} \alpha$ does not adequately trace the SFR in dwarfs, is not relevant to this work. Lee et al. (2009b) have also found that, whereas the dispersion in $\operatorname{SSFR}(\mathrm{H} \alpha)$ increases toward the lowest luminosities by $\sim 60 \%$, the increase is only $\sim 25 \%$ when SSFR(FUV) is adopted. Those authors interpreted this difference as evidence that UV measures are less affected by purely stochastic variations in the formation of high-mass stars.

Specifically presented for comparison with similar diagrams presented by other authors (e.g., Bothwell et al. 2009; Lee et al. 2011; Salim et al. 2007), Figure 12 presents various ways of examining the SF properties of the $s$-sed galaxies in the context of the parent $\alpha .40$-SDSS sample. The horizontal layout shows the run of stellar mass, $u-r$ color, and the $m_{\mathrm{NUV}}-r$ color, from left to right, respectively. In the vertical direction, from top to bottom, panels show the SFR, SSFR, and the birthrate parameter $b$. In all panels, the 74 colored symbols represent the galaxies in the s-sed ALFALFA dwarf sample; for those objects, physical properties are determined by SED fitting (see Section 4.1) to both the UV and optical magnitudes. In the left two columns, black contours and gray small points depict the distribution for the parent $\alpha .40$-SDSS sample in high and low number density regions, respectively, derived from SED fitting to the SDSS bands only. Because GALEX photometry is not examined here for the $\alpha .40$-SDSS sample, the right panels show only the ALFALFA dwarfs. Filled symbols show the less $\mathrm{H}$ i massive $s$-com galaxies (45), while open symbols denote the additional s-sup ones (29). Red squares identify galaxies that are members of the Virgo Cluster (17), while blue circles show all the others. Bigger symbols represent galaxies with SED fitting $\chi_{\text {best }}^{2}<10(46)$, while smaller ones have $\chi_{\text {best }}^{2}>10(28)$. Typical error bar estimates for the s-sed sample are plotted in the bottom left corners of Figures 12-14, with the same symbol definition in these plots. Note that the $m_{\mathrm{NUV}}-r$ range is scaled to be two times that of the $u-r$ color (refer to Figure 9).

In general, the parent $\alpha .40$ sample exhibits the known trends that the SFR increases with stellar mass and that a bimodal distribution in SFRs is evident at the highest stellar masses. However, as is clear in Figure 12, at low stellar masses, the SFR distributions fall below the extrapolation of the ridge defined by the massive systems. In particular, the ALFALFA s-sed dwarfs (the circles and squares) correspond to the systems of lowest stellar mass and define a low, broad tail of the SFR distribution. In the SFR versus $u-r$ color plot, while most of the ALFALFA dwarfs lie on the blue branch, the colors remain almost unchanged despite their varying, but relatively small, SFRs.

As shown also by Haynes et al. (2011), the ALFALFA catalog is clearly biased against red sequence galaxies. Still, the bimodal nature of the distributions of SSFR is evident in the left panel of the second row of Figure 12. At the high-mass end, a relatively small population of red sequence $\alpha .40$ galaxies occupies the low SSFR regime. The contours of the blue star-forming sequence reflect the general trend that the SSFR generally decreases with increasing stellar mass. At the same time, compared to that in the intermediate mass range $\left(10^{8}-10^{10} M_{\cup}\right)$, the dispersion of the SSFR distribution in a given stellar mass bin increases below $M_{*} \sim 10^{8} M_{0}$. The standard deviation of the log SSFR distribution for galaxies in a given $\log M_{*}$ bin is $0.55 \mathrm{dex}$ in $9.25<\log M_{*}<9.75,0.71$ dex in $7.75<\log M_{*}<8.25$, and increases to 0.83 dex in $6.75<\log M_{*}<7.25$. Most of the ALFALFA dwarfs lie in this low stellar mass regime, and not surprisingly, they show a large dispersion in SSFR. Moreover, because of the $\mathrm{Hi}$ mass cutoff used to define the ALFALFA dwarf sample, it actually includes galaxies with low $\mathrm{Hi}$ mass for their stellar mass, e.g., they are relatively gaspoor in comparison with the parent $\alpha .40$-SDSS sample (see Section 5.3). This selection tends to exaggerate the dispersion of the SSFRs to low values, and a concentration of small gray points from the parent sample with higher SSFRs is visible above the $s$-sed galaxies within the same $M_{*}$ range. For the same reason, the additional s-sup galaxies with their higher gas fractions have higher SSFRs on average than the lower Hi mass $s$-com ones. The few extreme outliers from the star-forming sequence with low SSFRs are mostly galaxies drawn from the lowest $\mathrm{H} \mathrm{i}$ mass $s$-com sample (filled circles and squares). Several of them 


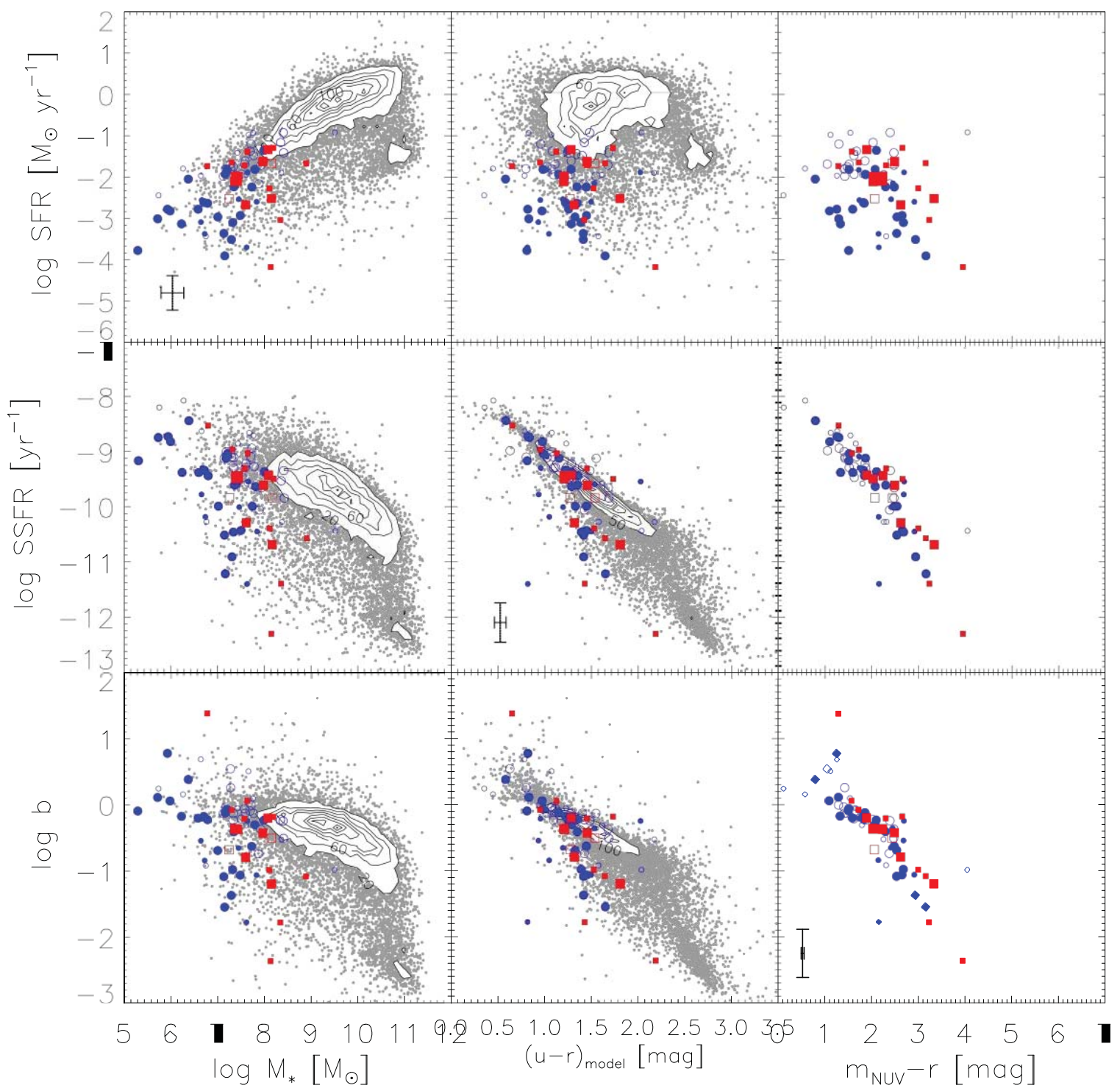

Figure 12. ALFALFA dwarfs on the star-forming sequence. Black contours and gray small points depict the distribution for the parent $\alpha .40$-SDSS sample, in high and low number density regions, respectively. All the other colored symbols represent the $s$-sed galaxies ( 74 of them) with reliable UV/optical magnitudes so that the SED fitting can be applied. Filled symbols belong to the s-com (45), while open ones to the s-sup sample (29); red squares denote Virgo Cluster members (17), while blue circles are outside of Virgo (57); bigger symbols represent galaxies with SED fitting $\chi_{\text {best }}^{2}<10$ (46), while smaller ones have $\chi_{\text {best }}^{2}>10$ (28). The typical error bars for the $s$-sed galaxies are plotted in the bottom left corners. The horizontal layout shows the run of stellar mass, $u-r$, and the $m_{\mathrm{NUV}}-r$ color. In the vertical direction we examine the SFR, SSFR, and the birthrate parameter $b$. Note that the $m_{\mathrm{NUV}}-r$ range is scaled to be two times that of the $u-r$ color (refer to Figure 9).

(A color version of this figure is available in the online journal.)

are dEs/dSphs in Virgo (e.g., AGC $220819=$ VCC 1617 and AGC 223913 = VCC 1649; see images of the latter shown in Figure 7), among the very few early-type Virgo dwarfs detected by ALFALFA (G. Hallenbeck et al. 2012, in preparation).

For galaxies with low SSFRs, a blind $\mathrm{Hi}$ survey is more efficient at the detection of galaxies at both the low and high stellar mass ends (S. Huang et al. 2012, in preparation). Moreover, a blind $\mathrm{H} \alpha$ survey would be biased against galaxies with low SSFRs. The sample analyzed by Bothwell et al. (2009) is heterogenous, making use of existing compilations of $\mathrm{Hi}$ and $\mathrm{H} \alpha$ data derived for other purposes. For example, the local low SSFR galaxies with low $M_{*}$ were particularly targeted for the 11HUGS program, which contributes significantly to the $\mathrm{H} \alpha$ data used by Bothwell et al. (2009). Similarly, the $\mathrm{H}$ i data analyzed in Bothwell et al. (2009) include a large population of massive cluster galaxies that have low SSFRs and were included in the targeted but deep surveys of nearby rich clusters
(Springob et al. 2005). ALFALFA probes to a slightly lower stellar mass range than the sample examined by Bothwell et al. (2009) and reveals outliers from star-forming sequence at both mass ends similar to those noticed by those authors. Thus, the breakdown of the star-forming sequence at the low-mass end is real and indicates that the stellar mass is no longer the dominant regulator of SF in dwarfs. It is possible that dwarfs are more vulnerable to environmental effects so that their gas supply can be easily disturbed and thus SF quenched. In the intermediate $M_{*}$ range, many outliers with extremely low SSFRs exist within the $\alpha .40$ parent sample, but their number density relative to the contoured region is so low that the dispersion of SSFRs remains low. However, while ALFALFA reveals that outliers with anomalously low SSFR for their stellar mass exist at all masses, intermediate-mass outliers are missing in similar plots (e.g., Figure 8) from Bothwell et al. (2009). Given the heterogenous sample that the Bothwell et al. (2009) analysis is 


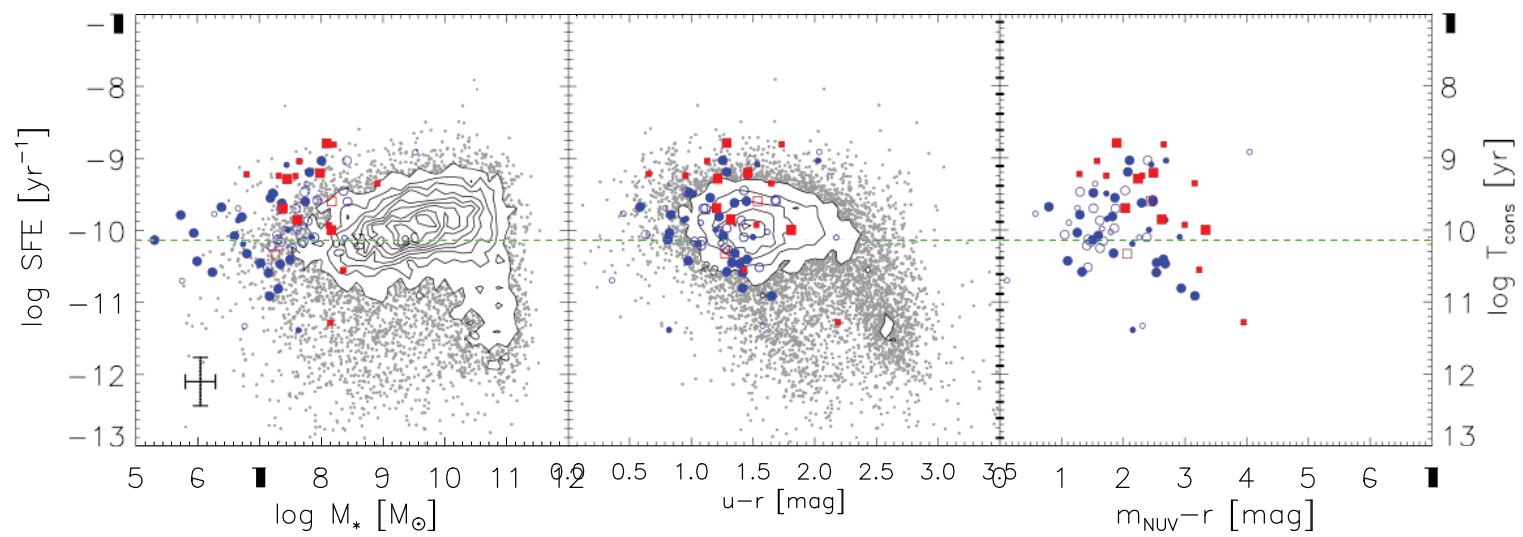

Figure 13. Star formation efficiency $\left(\mathrm{SFE}=\mathrm{SFR} / M_{\mathrm{H}}\right.$ i $)$ and gas depletion timescale $\left(T_{\text {cons }}=\mathrm{SFE}^{-1}\right)$. Symbol definition follows Figure 12 . The green dash-dotted line corresponds to SFE $=t_{\mathrm{H}}^{-1}$, where $t_{\mathrm{H}}$ is the Hubble time. Left: SFE against stellar mass. The SFEs of ALFALFA detections are on average lower than optically selected galaxies. The averaged SFEs in each stellar mass bin increase slowly with stellar mass, with the SFE $\sim t_{\mathrm{H}}^{-1}$ at the low-mass end. Selected to have lower $\boldsymbol{f}_{\text {gas }}$ compared to the $\alpha .40$-SDSS galaxies in this stellar mass range, the s-sed galaxies have higher SFEs compared to the general trend if extrapolated into this stellar mass range. A large portion of them even have $T_{\mathrm{cons}},: \mathrm{S} t_{\mathrm{H}}$. In particular, the majority of Virgo members in the s-sed sample have $T_{\text {cons }}, \mathrm{S} t_{\mathrm{H}}$. Middle and right: the SFE is seen to vary very little with color in either the optical or optical UV bands.

(A color version of this figure is available in the online journal.)

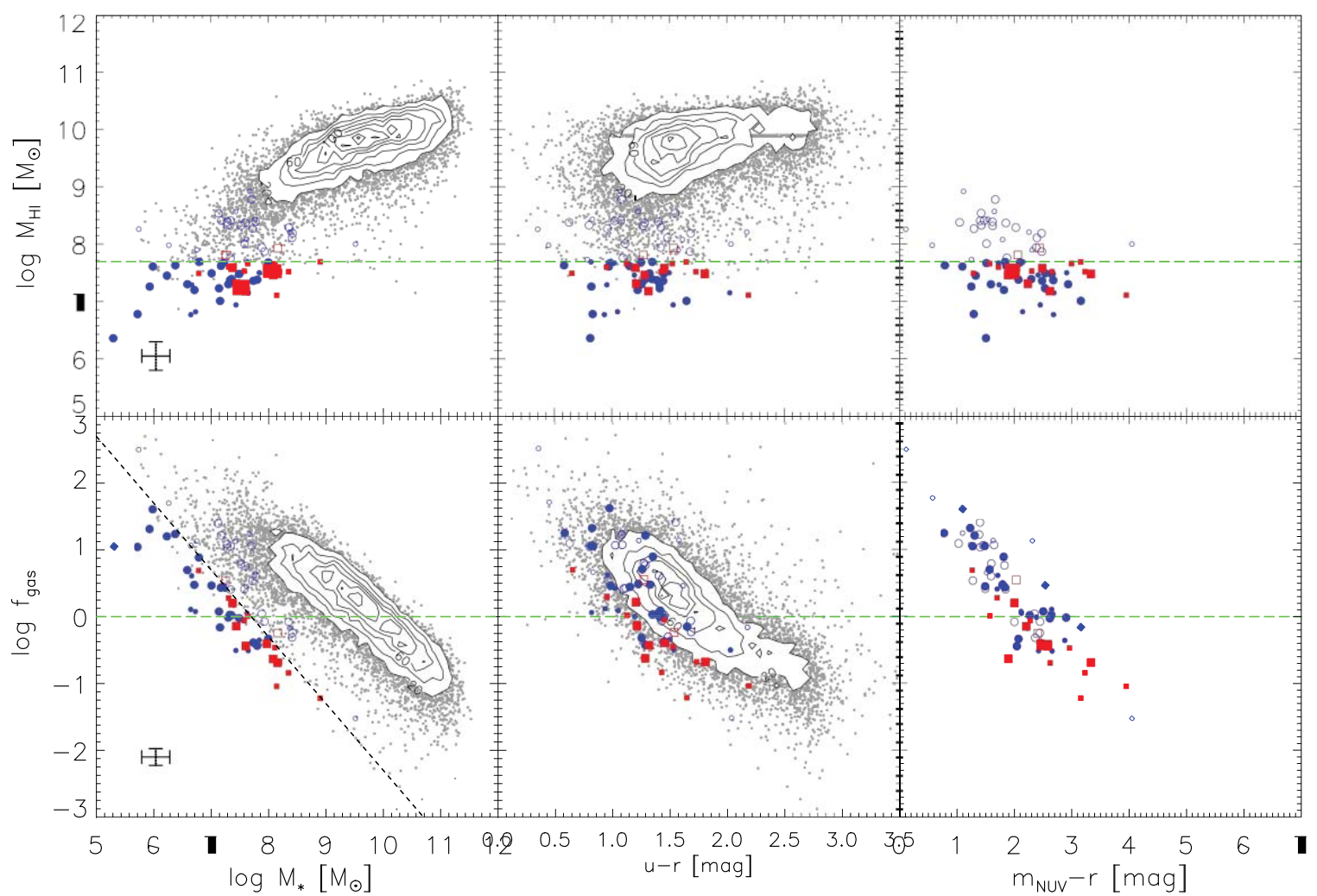

Figure 14. Hi mass and gas fraction, $f_{\text {gas }}=M_{\mathrm{H} \text { i }} / M_{*}$. Symbol definition follows Figure 12. Upper row: $M_{\mathrm{H} \text { i }}$ as a function of stellar mass and colors. The dashed line corresponds to the cutoff in $M_{\mathrm{H}}$ i of the $s$-com sample. The $M_{\mathrm{H}}$ increases with $M_{*}$, with the $s$-sed lying on the low mass tail. At a given $M_{\mathrm{H}}$, Virgo members (red squares) have on average lower $f_{\text {gas }}$. Bottom row: $f_{\text {gas }}$ as a function of the three. The dashed line corresponds to $M_{\mathrm{H}} \mathrm{i}=M_{*}$, while the black dash-dotted line shows again $M_{\mathrm{H} \mathrm{i}}=10^{7.7} M_{\mathrm{U}}$. Because ALFALFA is biased against gas-poor galaxies, the correlation that $\boldsymbol{f}_{\text {gas }}$ decreases with increasing $M_{*}$ is clean. Specifically, the $\mathrm{H}$ i mass selection of the ALFALFA dwarf sample results in low $f_{\text {gss, }}$, relative to the $\alpha .40$-SDSS in this stellar mass range. However, 46 of the $74 s$-sed ALFALFA dwarfs
still have $f$ gas $>1$. For galaxies blueward of $u-r=2, \mathcal{f}_{\text {gas }}$ is lower in the redder ones.

(A color version of this figure is available in the online journal.)

based on, the absence of low SSFR galaxies in the intermediatemass range could be due to some combined bias in the sample selection criteria by the dual requirement of $\mathrm{H} \alpha$ and $\mathrm{H} \mathrm{i}$ (from the Cornell digital $\mathrm{H} \mathrm{i}$ archive, selected typically by luminosity, optical size, and/or cluster membership) measures.

The contours in the SSFR versus $u-r$ diagram (middle column) show that the SSFRs of $\alpha .40$ galaxies are tightly correlated with the $u-r$ color: for $\mathrm{Hi}$-selected galaxies, nearly all of which are blue, the SSFRs, and thus the recent SFHs, are well constrained by the $u-r$ color itself. Given the fact that the NUV band better traces SF than the $u$ band, we would have expected that the $m_{\mathrm{NUV}}-r$ color would be better correlated with SSFR than is the $u-r$ color. However, such an improvement is not obvious in the right panel: the larger attenuation correction 
introduces a greater dispersion in the observed NUV, so that a better correlation between SSFRs and $m_{\mathrm{NUV}}-r$ colors is in fact not clearly evident among the $s$-sed dwarf galaxies.

Finally, we consider another quantity related to the SSFR, namely, the birthrate or $b$-parameter, $b=$ SFR (SFR) $=$ $\left(\mathrm{SFR} / M_{*}\right) T R$, where $T$ is the age of the galaxy and $R$ is the fraction of the mass formed over the galaxy's lifetime that does not eventually get returned to the ISM or intergalactic medium (Brinchmann et al. 2004). A typical value of $R$ is $\sim 0.5$ (Brinchmann et al. 2004), and on the assumption that all galaxies started forming stars shortly after the big bang, $T \sim 13$ Gyr. For these choices of $T$ and $R$, a galaxy with $b=1$, i.e., a constant SFR, would have an SSFR of $10^{-9.8} \mathrm{yr}^{-1}$. Thus, galaxies with SSFRs larger than this value appear to have current SFRs above their lifetime average. With the caveat that SFR measures based on $\mathrm{H} \alpha$ emission in the low SFR regime may be systematically underestimated, Lee et al. (2009b) found that only $\sim 6 \%$ of low-mass 11 HUGS galaxies are currently experiencing massive global bursts ( $\mathrm{H} \alpha$ EW above $100 \AA$ or $b \geqslant 2.5)$. Here, we find that 13 galaxies $(18 \%)$ of the ALFALFA $s$-sed sample have $b>1$ and five galaxies (7\%) even have $b>2.5$. Because of the relationship between the SSFR and the $b$-parameter, the distributions of $\log b$ versus stellar mass, $u-r$, and $m_{\mathrm{NUV}}-r$ colors, shown in the bottom row of Figure 12, resemble the corresponding distributions in the second row, suggesting that dwarfs have both higher SSFRs and $b$-parameters, on average, compared to higher mass galaxies. For this reason, it is even more essential to take bursty behavior into account when modeling the SEDs of $\mathrm{H}$ i-selected dwarfs. At the same time, the equivalent widths of emission lines, e.g., $\mathrm{H} \alpha$, which also characterize the SSFRs or $b$-parameters (Lee et al. 2009b), could be large enough to modify the continuum emitted by the underlying stellar population, leading to large $\chi^{2}$ in the SED fitting (Section 4.1). Despite the caveats, the wide spread in $b$ values evident among the $s$-sed sample suggests that the SFHs of the ALFALFA dwarfs are highly variable and

no longer a strong function of $M_{*}$. This result suggests further that other factors including environmental effects may play an important role in the regulation of SF in $\mathrm{H}$ i-selected dwarfs.

\subsection{Star Formation Efficiency and the Gas Depletion Timescale}

In addition to normalizing the SFRs by stellar mass to infer the level of the current SFR relative to its past average, we can also normalize the SFR by the $\mathrm{H}$ i mass to infer the ratio relative to potential future SF, as inferred from the present amount of $\mathrm{Hi}$ gas. Following Schiminovich et al. (2010), we define the $\mathrm{SF}$ efficiency as $\mathrm{SFE}=\mathrm{SFR} / M_{\mathrm{H}}$ i and its reciprocal as the gas consumption timescale $T_{\text {cons }}=M_{\mathrm{H}}$ /SFR, also known as the Roberts time (Roberts 1963). Observationally, low SFEs have been measured for low-mass galaxies and LSB galaxies (Boissier et al. 2008), and conversely, high efficiencies have been measured in starburst galaxies and interacting systems.

Based on a sample of $\sim 1000$ local galaxies $10^{7} M_{v} \diamond M_{*} \vartheta$ $10^{11} M_{U}$, with $\mathrm{Hi}$ and $\mathrm{H} \alpha$ fluxes compiled from the literature, Bothwell et al. (2009) found that, with increasing luminosity, the $\mathrm{H}$ i gas fraction $M_{\mathrm{H}} / M_{*}$ drops off faster than the $\mathrm{H} \alpha$-derived SFR, resulting in shorter $T_{\text {cons }}$ for more luminous galaxies. Those authors report $T_{\text {cons }}$ in excess of several Hubble times $\left(t_{\mathrm{H}}\right)$ for samples of dwarf irregular galaxies. In contrast, using the FUV luminosity instead of $\mathrm{H} \alpha$ to measure the SFR, Lee et al. (2011) found that dwarf galaxies may not be as drastically estimates, with SFEs exceeding $t_{\mathrm{H}}^{-1}$. Although the Bothwell et al. (2009) sample also exploits $\mathrm{H}$ i measures for a large sample to derive gas fractions, it is based on optical, rather than $\mathrm{Hi}$, selection, deriving most of its $\mathrm{H}$ i properties from the extensive compilation of $\mathrm{Hi}$ spectra contained in the Cornell digital $\mathrm{Hi}$ archive (Springob et al. 2005). In fact, it is important to note that more than $2 / 3$ of ALFALFA $\mathrm{Hi}$ detections are new and were not included in any of the extensive pointed observations that contributed to the digital archive data set (Haynes et al. 2011). In terms of the availability of $\mathrm{H}$ i measures, the $\alpha .40$ parent sample is more homogeneous and its size is more than 10 times larger than the Bothwell et al. (2009) sample. Moreover, we rely on SED fitting to derive physical quantities, rather than deriving stellar masses based on the $M / L$ versus color relation (see Section 4.2) or the $\mathrm{H} \alpha$ luminosity for the SFR (see Section 4.5). In addition, the ALFALFA dwarf population used here extends to lower $\mathrm{H}$ i masses compared to those in Lee et al. (2011). Therefore, the ALFALFA-based galaxy sample is more comprehensive than either of those two previous studies in terms of both probing the general trends exhibited by $\mathrm{Hi}$ selected galaxies and exploring the behavior of the lowest mass dwarfs.

Figure 13 shows the distribution of SFE versus $M_{*}, u-r$, and $m_{\mathrm{NUV}}-r$ colors for the ALFALFA samples. Contours and gray small points in the left two panels depict the distribution of the full $\alpha .40$-SDSS data set, and the symbol definition follows the same convention as in Figure 12. The superposed dashdotted line corresponds to $\mathrm{SFE}=t_{\mathrm{H}}^{-1}$. Three main conclusions can be drawn from the general distribution. First, as discussed more fully elsewhere (S. Huang et al. 2012, in preparation), ALFALFA galaxies have, on average, higher SFRs but lower SFEs than optically selected ones, due to their higher than average $\mathrm{Hi}$ masses. At the same time, the SFEs are barely dependent on either the $u-r$ or $m_{\mathrm{NUV}}-r$ color as shown in the center and right panels of Figure 13. Second, although the variation in the average SFR is mild across the stellar mass range of $10^{8}-10^{11} M_{\cup}(\delta \log (\mathrm{SFE}) \sim 0.5 \mathrm{dex})$, the averaged SFE in each stellar $\mathrm{m}$ bin increases with stellar mass. At the lowass

mass end, the SFE $\sim t_{\mathrm{H}}^{-1}$, albeit with considerable scatter. Third, a population of galaxies with very low SFE, arising mainly because of their low SFR (see Figure 12), stands distinct from the general distribution at the high-mass end $\left(M_{*} \diamond 2 \times 10^{10} M_{0}\right)$.

Notably, despite the fact that they are selected to have the lowest $\mathrm{H}$ i masses in the $\alpha .40$ catalog, the $s$-sed dwarfs (circles and squares) typically have higher SFEs regardless of their low SFRs, compared to an extrapolation of the general trend into this stellar mass range. In contrast to the extremely low SFEs derived from $\mathrm{H} \alpha$ emission for the dwarfs studied by Bothwell et al. (2009), the ALFALFA dwarfs studied here are forming stars efficiently, and a large portion of them even have $T_{\text {cons }} \geqslant t_{\mathrm{H}}$. In particular, 14 out of 17 Virgo dwarfs (red squares) detected by ALFALFA have $T_{\text {cons }}<t_{\mathrm{H}}$, suggesting that the cluster environment may actually play a role in enhancing their SFEs by either accelerating their evolution and thus driving them to the gas-poor side or simply stripping part of their gas away. inefficient in converting gas into stars as suggested by $\mathrm{H} \alpha$ 
The Astronomical Journal, 143:133 (28pp), 2012 June

In contrast, AGC 223913 and AGC 220819, both dE/dSphs in Virgo, have low SFEs and $T_{\text {cons }}>t_{\mathrm{H}}$.

\subsection{Hi Mass and Gas Fraction}

As specified in Section 3.4, we define the gas fraction here simply as the ratio of the $\mathrm{Hi}$ mass to the stellar mass

$\left(f_{\text {gas }}=M_{\mathrm{H} \mathrm{i}} / M_{*}\right)$, without a correction for the presence of helium or for other phases of hydrogen. Little is actually known 
about the molecular content of dwarf galaxies other than that it is low. Numerous other studies, e.g., Walter et al. (2007), have shown that dwarf galaxies, including ones detected in $\mathrm{H} \mathrm{i}$, are notoriously difficult to detect in $\mathrm{CO}$ and contain only small masses of dust. Moreover, the relationship between $\mathrm{CO}$ and $\mathrm{H}_{2}$ may break down in galaxies of low metallicity. Since the fraction of the ISM contributed by very cold molecular material is likely to be insignificant (e.g., Leroy et al. 2007) and the uncertainty in it large, we assume that the ALFALFA dwarfs are likely dominated by $\mathrm{Hi}$ at least on global scales and equate the $\mathrm{Hi}$ mass with their total gas mass.

Figure 14 presents the distribution of $\mathrm{Hi}$ mass (upper panels) and gas fraction $f_{\text {gas }}$ (lower panels) as a function of stellar mass, $u-r$ and $m_{\mathrm{NUV}}-r$ colors, respectively. As before, in the left two panels, the ALFALFA dwarf galaxies in the $s$-sed sample are shown as circles and squares superposed on the parent $\alpha .40-$ SDSS sample (black contours and gray points). The horizontal dashed line in the upper panels marks the $\mathrm{Hi}$ mass cutoff $M_{\mathrm{H} \mathrm{i}}<10^{7.7} M_{\cup}$ imposed on the $s$-com sample. In the lower plots, the horizontal dashed line traces $M_{\mathrm{H} \mathrm{i}}=M_{*}$, while in the lower left panel, the diagonal dash-dotted line traces the locus where $M_{\mathrm{Hi}}=10^{7.7} M_{\mathrm{u}}$. The distributions for the full ALFALFA population are smooth, with the mean $M_{\mathrm{H} \mathrm{i}}$ increasing with $M_{*}$, and the mean $f_{\text {gas }}$ decreasing monotonically as $M_{*}$ grows. Unsurprisingly, $\mathcal{f}_{\text {gas }}$ is tightly correlated with both the $u-r$ and $m_{\mathrm{NUV}}-r$ colors: bluer galaxies have higher gas fractions. Note again that ALFALFA probes significantly lower masses, both stellar and $\mathrm{H}$ i, than other surveys. Imposing a limit in $M_{\mathrm{H}}$ i confines the ALFALFA $s$-com sample to fall below the horizontal dashed line in the upper panels and to the left of the dash-dotted line in the bottom left panel.

As evident in the bottom row of panels, some of the lowest mass galaxies have extremely high gas fractions $\left(f_{\text {gas }}>100\right)$. Visual inspection of the most egregious cases shows that these values are corrupted by poor SDSS photometry or, possibly, by the assignment of the wrong OC. Although we have checked the photometry for the dwarf sample individually, only a portion of the OCs in the $\alpha .40$-SDSS sample has been inspected individually to set an SDSS photometric quality flag (Haynes et al. 2011). As a result, it is not surprising that some of the $\alpha .40$ galaxies with $f_{\text {gas }}>100$ suffer from shredding, with a fainter child assigned as the SDSS cross-match and thus producing an underestimate of $M_{*}$. In other cases, confusion within the ALFA beam may result in a match to the wrong object, albeit the "most probable" OC. The complexities and caveats involved in assigning OCs to the ALFALFA data set are discussed in Haynes et al. (2011). However, some dwarfs really do appear to be highly $\mathrm{H}$ i dominated. For example, AGC 174514 in the supplementary s-sup sample also has an $f_{\text {gas }}>100$; its images are shown in Figure 7. It is the bluest galaxy among the $s$-sed sample, suggesting that it is truly a galaxy with lots of gas and not many stars. However, it may not be a single object and the $f_{\text {gas }}$ could be a slight overestimate due to the uncertainty of the distribution of the $\mathrm{Hi}$; there are several additional small blue LSB clumps within the ALFALFA beam that may contribute comparable optical luminosities, thereby raising the stellar mass by some factor, but certainly not $10^{2}$; this galaxy remains an unusual object with a very high gas fraction.

Using a similar $f_{\text {gas }}$ versus $M_{B}$ diagram, Bothwell et al. (2009) found a population of outliers, namely, early-type galaxies with extremely low $f_{\text {gas }}<0.05$, extending below the main distribution (Figure 3 of that paper) at the bright end $\left(M_{B}<\right.$ $-18)$. They further identified this phenomenon with the large spread in the SSFRs of massive galaxies, suggesting that a dearth of fuel for SF causes the extremely low SSFRs. In comparison with Figure 3 of Bothwell et al. (2009), Figure 14 reveals no concentration of galaxies with low $\mathcal{f}_{\text {gas }}$ below the main distribution at the high-mass end, despite the existence of many low SSFR massive galaxies. The absence of very low $\mathcal{f}_{\text {gas }}$ massive galaxies in ALFALFA is not surprising given its wellcharacterized flux completeness and near-constant integration time. In contrast, some of the $\mathrm{H}$ i measures contributed by the Springob et al. (2005) data set and used by Bothwell et al. (2009) made use of very deep targeted observations designed specifically to probe to very low gas fractions in massive earlytype and cluster galaxies. The desire to probe the true range of gas fractions representative of the most massive galaxies likewise motivated the targeted deep observations of the GASS program (Catinella et al. 2010).

At the same time, they found that the population of low-mass galaxies with a large spread in SSFRs is not matched by an equivalent shift to a larger range of $\mathcal{f}_{\text {gas }}$. On the other hand, the broadening of the $f_{\text {gas }}$ distribution at the low-mass end is more evident in the ALFALFA data set, compared to that seen by Bothwell et al. (2009). Among the 176 ALFALFA dwarfs in the low Hi mass $s$-com set, only two are included in the Springob et al. (2005) catalog. Hence, it is not surprising that Bothwell et al. (2009) see no broadening of the $f_{\text {gas }}$ distribution at the low-mass end. $\mathrm{H}$ i selection yields a high overall gas fraction across the full range of sampled masses and, at the same time, still a larger dispersion in the SSFR distribution at both the high and low stellar mass ends (see Section 5.1).

In Figure 14, the ALFALFA dwarfs in the s-sed sample (circles and squares) lie on the low-mass tail of both the $M_{\mathrm{H} \text { i }}$ and $f_{\text {gas }}$ distributions: at low stellar masses, the ALFALFA dwarfs probe a relatively low gas fraction population. This departure from the norm for the majority of the ALFALFA detections results from the imposition of the low $\mathrm{H}$ i mass cutoff. Among the low-mass $s$-com galaxies (filled symbols), at a given $M_{\mathrm{H}}$, the Virgo members (red squares) have, on average, higher $M_{*}$ and thus lower gas fractions than the non-Virgo ones (blue circles). Given the general trend between $\mathcal{f}_{\text {gas }}$ and $M_{*}$, the most gasrich galaxies have very low $M_{*}$. Even if their $f_{\text {gas }}>1$, the least massive may lie below the $\mathrm{H}$ i sensitivity of ALFALFA at the Virgo distance $\left(\log M_{\mathrm{H}} \sim 7.3\right)$. Alternatively, the low gas fractions may reflect a possible connection between the cluster environment and relative gas-poorness; in fact, G. Hallenbeck et al. (2012, in preparation) show that some morphologically early Virgo dwarfs contain "normal" gas fractions and argue that they must have recently accreted their $\mathrm{Hi}$. Here, as is seen in Figure 14, more than half (46) of the s-sed galaxies have $f_{\text {gas }}>1$, meaning that their baryonic mass is dominated by their atomic gas, rather than by their stars.

\subsection{The Star Formation Law and the Global Interplay of Gas and Stars}

It is well known that the local surface density of the SFR is strongly correlated with the local surface density of gas, $\Sigma_{\text {SFR }} \propto \Sigma_{\text {gas }}^{\alpha}$, at least when averaged over $\sim$ kpc scales. A precise measurement of its exponent is the "Kennicutt-Schmidt law" (Kennicutt 1998b),

$$
\log \left(\Sigma_{\mathrm{SFR}}\left[M_{\mathrm{O}} \mathrm{yr}^{-1} \mathrm{kpc}^{-2}\right]\right)=\alpha \log \left(\Sigma_{\mathrm{gas}}\left[M_{\mathrm{O}} \mathrm{pc}^{-2}\right]\right)+C,
$$

where $C=\log \left[(2.5 \pm 0.7) \times 10^{-4} / 1.5\right], \alpha=1.4 \pm 0.15$, and $\Sigma_{\text {gas }}=\Sigma_{\mathrm{Hi}}+\Sigma_{\mathrm{H}_{2}}$. The denominator of $C, 1.5$, results from the 
(a)

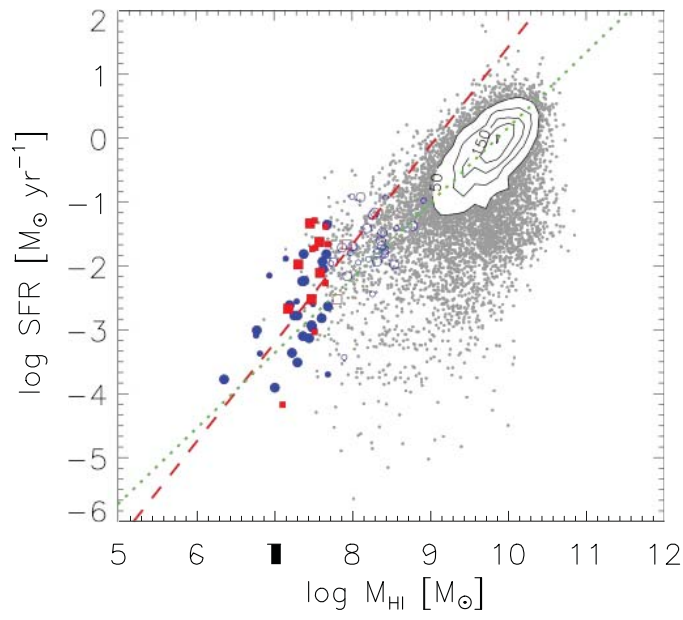

(c)

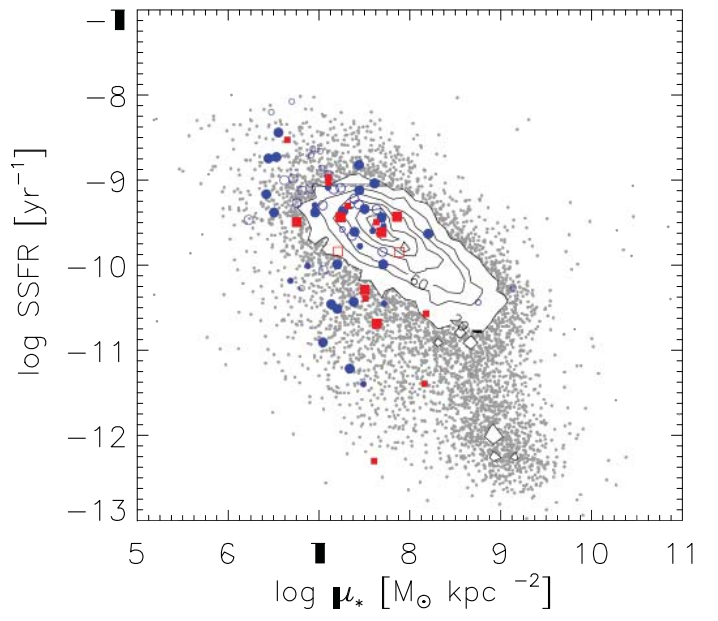

(b)

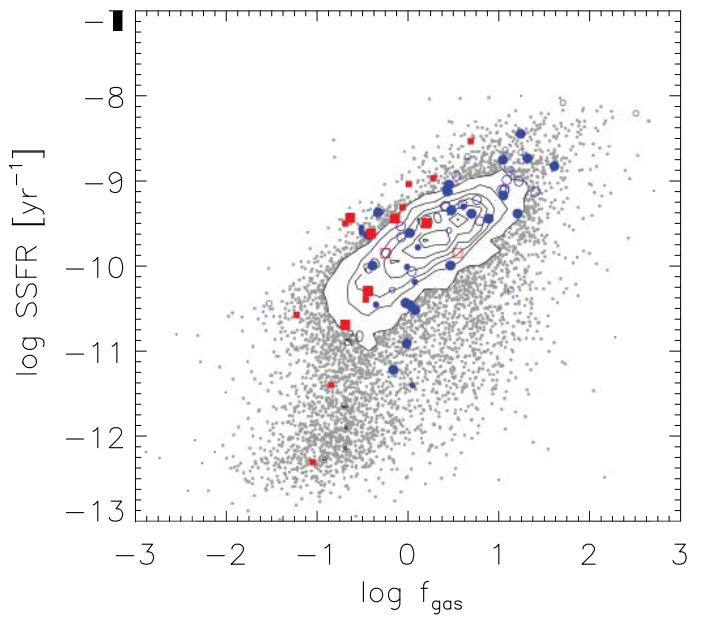

(d)

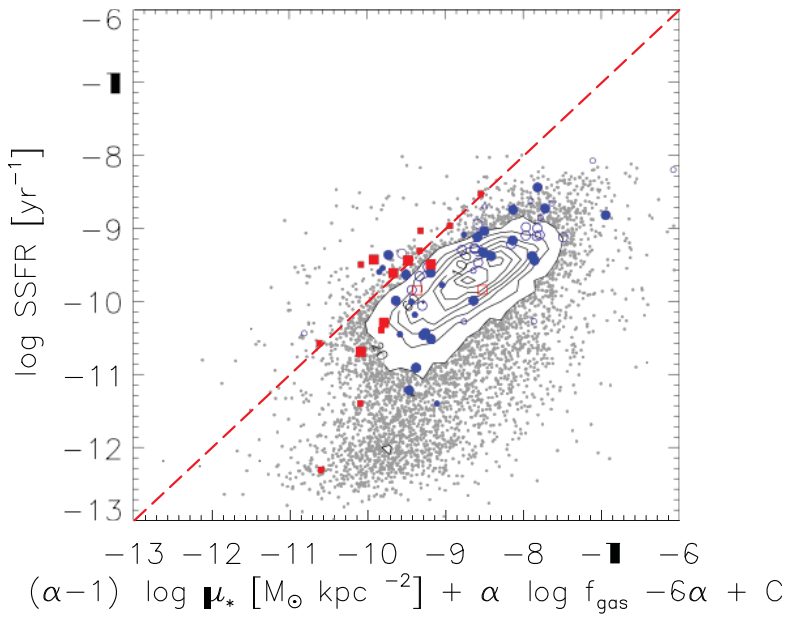

Figure 15. Gas content and star formation. Symbol definition follows Figure 12. (a) The SFR increases with the Hi mass. The dotted line has a slope of 1.2, corresponding to the linear fit to all the $\alpha .40$-SDSS galaxies. The dashed line, of slightly steeper slope 1.5 , is the linear fit to the $s$-sed only. (b) Both axes in panel (a) are normalized by the stellar mass. Besides the general trend that the SSFR increases with the gas fraction, the SSFRs of a subset of galaxies drop dramatically once the gas fraction is below $\sim 0.3$. (c) SSFRs decrease with increasing stellar surface mass density, $\mu_{*}$. s-sed have relatively low $\mu_{*}$. (d) By assuming that the aperture with a radius of $r_{50, z}$ also contains half of the stellar mass, SFR, and Hi mass, the star formation law (Kennicutt 1998b) is substituted into the expression of SSFR, resulting in the quantity on the $x$-axis. The adopted values of the scaling factor $C$ and exponent $\alpha$ reflect the Kennicutt (1998b) result. Although a clear correlation appears, the main distribution is shifted to the right of the dashed one-to-one line.

(A color version of this figure is available in the online journal.)

Chabrier IMF adopted in this work. Such a super-linear Schmidt law $(\alpha>1)$ suggests a SF efficiency that rises with gas surface density. Measurements of azimuthally averaged gas and SFR profiles show that the SFR correlates better with the molecular hydrogen component than with the total gas density at least within the optical disk (Bigiel et al. 2008). Yet, on global scales averaged over the whole galaxy, SFRs appear to correlate better with the total gas $\left(\mathrm{Hi}+\mathrm{H}_{2}\right)$, rather than the molecular gas, surface density (Kennicutt 1998b). Global quantities such as those available to us sample a range of gas surface densities, timescales, and conditions within the ISM, and since we have only global $\mathrm{Hi}$ measures, we can only estimate $\Sigma_{\text {gas }}$ by proxy, using the global $\mathrm{H}$ i mass and the optical size of the stellar disk.

Albeit crude in comparison to more detailed studies of spatial resolved atomic and molecular distributions, we examine via Figure 15 the correlations of global quantities related to the Schmidt law to demonstrate the constraints placed by the global $\mathrm{Hi}$ measures on global SFRs. As before, both the $\alpha .40$ and the dwarf s-sed samples are shown, with symbol definitions following Figure 12. Panel (a) of Figure 15 shows the distribution of the SFR as a function of $M_{\mathrm{H} i}$; this can be thought of as the global atomic and volumetric SF law. The green dotted line with a slope of 1.2 is the linear fit to all the $\alpha .40-$ SDSS galaxies. The dashed line, of slightly steeper slope 1.5 , is the linear fit to the galaxies in the dwarf s-sed sample only. While it is natural to expect higher SFRs in galaxies with more gas available to form stars, the ALFALFA $s$-sed dwarf galaxies define the low $\mathrm{Hi}$ mass tail of the distribution of star-forming galaxies. Note also the presence of a second tail exhibited by the parent $\alpha .40$-SDSS population at very low SFRs; these galaxies may include a population of objects whose $\mathrm{Hi}$ distributions extend beyond their star-forming disks. In Section 5.2, we have already presented the result that the SFE increases slowly with $M_{*}$. In fact, other recent works demonstrate that the $\Sigma_{\mathrm{SFR}}-\Sigma_{\text {gas }}$ relation steepens at low gas surface densities due to the transition from atomic to molecular hydrogen (e.g., Bigiel et al. 2008). The dependence of SFR on $M_{\mathrm{H}}$ for the ALFALFA dwarfs shown in panel (a) shows a suggestive mild steepening at low $M_{\mathrm{H} \text { i }}$. Similarly, Roychowdhury et al. (2009) found that the SFR is generally lower in gas-rich dwarfs than predicted by the 
"Kennicutt-Schmidt law." Again, we note that the linkage of the scaling between SFR and $M_{\mathrm{H}}$ i with the power law derived for $\Sigma_{\mathrm{SFR}}$ and $\Sigma_{\text {gas }}$ is subject to various uncertainties, including the contribution from molecular gas and the relative extent of the star-forming and $\mathrm{Hi}$ disks. Therefore, this comparison of global quantities yields only a suggestive empirical result.

In panel (b), we normalize both axes by $M_{*}$, giving the relationship between SSFR and $f_{\text {gas }}$. Normalization by $M_{*}$ moves the galaxies in the ALFALFA dwarf $s$-sed sample from the tail seen in panel (a) back to within the main distribution. For the majority of ALFALFA galaxies, except those with extremely low SSFR, a clear trend exists: the more gas-rich (higher gas fraction), the higher is the SFR relative to the accumulated stellar mass (higher SSFR).

Given the fact that the majority of $\mathrm{Hi}$ disks are un-resolved by ALFALFA, we cannot quantify $\Sigma_{\text {gas }}$ directly. However, we can characterize the surface densities by introducing an arearelated quantity: the stellar surface mass density, $\mu_{*}$. Following Schiminovich et al. (2010), we define the stellar surface mass density:

$$
\mu_{*}\left[M_{0} \mathrm{kpc}^{-}{ }^{-}\right]=\frac{0.5 M_{*}\left[M_{0}\right]}{\pi\left(r_{50, z}[\mathrm{kpc}]\right)^{2}},
$$

where $r_{50, z}$ is the Petrosian half-light radius according to the SDSS pipeline measurements. Panel (c) of Figure 15 shows that SSFR decreases with increasing $\mu_{*}$ in general and that the dwarf $s$-sed galaxies, on average, have lower $\mu_{*}$ than the full ALFALFA population. For comparison with other studies, we adopt an aperture of radius $r_{50, z}$ and assume that, by definition, it encloses also one-half of the stellar mass. Although it is likely not a correct assumption, we assume first that this aperture also contains exactly half of the global SFR, as well as half of the $\mathrm{H}$ i mass, and thus

$$
\begin{aligned}
\log \left(\Sigma _ { \mathrm { SFR } } \left[M_{\left.\left.0 \mathrm{yr}^{-} \mathrm{kpc}\right]\right)}{ }^{-2}\right.\right. & \log \frac{1}{0.5 \mathrm{SFR}\left[M_{0} \mathrm{yr}^{-}\right]} \\
\log \left(\Sigma_{\mathrm{gas}}\left[M_{\mathrm{O}} \mathrm{pc}^{-2}\right]\right) & =\log \left(\sum_{\mathrm{gas}}\left[M_{0, z}[\mathrm{kpc}]\right)^{2}\right. \\
& =\log \frac{0.5 M_{\mathrm{H} \mathrm{i}}\left[M_{\mathrm{O}}\right]}{\pi\left(r_{50, z}[\mathrm{kpc}]\right)^{2}}-6
\end{aligned}
$$

The SF law averaged within this aperture can be re-written from Equation (3) as

$$
\begin{aligned}
\log \left(\Sigma_{\mathrm{SFR}}\left[M_{\cup} \mathrm{yr}^{-1} \mathrm{kpc}^{-2}\right]\right)= & \alpha\left(\log \mu_{*}\left[M_{\cup} \mathrm{kpc}^{-2}\right]-6\right) \\
& +\alpha \log f_{\mathrm{gas}}+C .
\end{aligned}
$$

Certainly the adoption of the right-hand side of Equation (4) to infer $\Sigma_{\text {SFR }}$ is crude at best. It is true or at least likely that (1) SF may be more concentrated than the older population (see Section 3.2), so that $\Sigma_{\text {SFR }}$ is underestimated; (2) the $\mathrm{Hi}$ disk is more extended than the stellar one so that $\Sigma_{\text {gas }}$ and thus $\Sigma_{\mathrm{SFR}}$ are both overestimated; (3) ignoring the contribution of $\mathrm{H}_{2}$ to the $\Sigma_{\text {gas }}$ also leads to an underestimate of $\Sigma_{\text {SFR }}$.

With the above caveats, Equation (4) gives a crude relation between the SSFR, $\mu_{*}$, and $f_{\text {gas }}$ :

$$
\begin{aligned}
\log \operatorname{SSFR}\left[\mathrm{yr}^{-1}\right]= & \log \frac{0.5 \mathrm{SFR}\left[M_{0} \mathrm{yr}^{-1}\right]}{\pi\left(r_{50, z}[\mathrm{kpc}]\right)^{2}}-\log \frac{0.5 M_{*}\left[M_{\mathrm{O}}\right]}{\pi\left(r_{50, z}[\mathrm{kpc}]\right)^{2}} \\
= & (\alpha-1) \log \mu_{*}\left[M_{\mathrm{O}} \mathrm{kpc}^{-2}\right] \\
& +\alpha \log \mathcal{f}_{\mathrm{gas}}-6 \alpha+C
\end{aligned}
$$

Adopting the values of $C$ and $\alpha$ from Equation (3), we plot panel (d) and the left-hand side (log SSFR) on the $y$-axis. Although these two quantities are correlated as expected with a slope of $\sim 1$, the main distribution is shifted from the one-to-one correlation, represented by the red dashed line, in the sense that the right-hand side overestimates the SSFR, i.e., $\Sigma_{\text {SFR }}$. Among the three possible caveats to the adoption of the right-hand side of Equation (4) to infer $\Sigma_{\mathrm{SFR}}$ that might lead to systematic errors in the $\Sigma_{\text {SFR }}$ estimates, only the second one, that the $\mathrm{Hi}$ gas extends beyond the stellar disk, would yield a shift in the correct direction. Additional factors that might cause such a shift include (4) the ALFALFA galaxies have sub-solar metallicity on average, requiring an even smaller $C$ (see Section 4.4); (5) because they are gas-rich in general, they also have lower SFEs relative to the typical SDSS galaxies (see Section 5.2) and thus smaller $C$ values. Both these interpretations are consistent with what is evident in Figure 15 panel (d), i.e., the standard $C$ (Kennicutt 1998b) may be an overestimate for an $\mathrm{Hi}$-selected population. Furthermore, a plot with the same $x$ - and $y$-axes as in Figure 15(d) but color coded by the mean SFEs of galaxies within each grid cell clearly shows that the offset from the oneto-one line increases with decreasing SFE.

Rather than adopting $C$ and $\alpha$ values from the Kennicutt-Schmidt law, Zhang et al. (2009) calibrated an empirical estimator of $\mathcal{f}_{\text {gas }}$ from SSFR and $\mu_{*}$, reformulated as

$$
\log \mathrm{SSFR}=2.96 \log \mu_{*}+3.85 \log \mathcal{f}_{\mathrm{gas}}-32.81
$$

corresponding to larger $\alpha$ and smaller $C$ values than given in Equation (3). Their best estimator of $f_{\text {gas }}$ had SSFR substituted by $g-r$ color, since optical colors can be good proxies for SSFRs (see Section 5.1). In agreement with Zhang et al. (2009), we also prefer a smaller SFE scaling factor than the Kennicutt (1998b) value (see Equation (3)). However, Zhang et al. (2009) calibrate their $f_{\text {gas }}$ estimator from an SDSS-selected sample with

existing $\mathrm{Hi}$ data and therefore relatively gas-poor compared to the ALFALFA $\mathrm{Hi}$-selected population. For this reason, their estimator systematically underpredicts the $f_{\text {gas }}$ of the $\alpha .40-$ SDSS galaxies by $\sim 0.3$ dex.

Panel (d) of Figure 15 shows that, compared to the full $\alpha .40$ distribution, the ALFALFA dwarf galaxies appear slightly closer to the one-to-one line given by Equation (5). Again, this result may arise from their selection as the lowest $M_{\mathrm{H}}$ i systems. As we have seen, the dwarfs studied here are relatively gas-poor (see Section 5.3) and have higher SFEs (see Section 5.2) compared to those estimated for the full $\alpha .40$ population in the same stellar mass range. Therefore, the fact that the dwarfs lie closer to the one-to-one relation traced by Equation (5) is consistent with the finding above that lower than typical SFEs drive the overall ALFALFA distribution to lower SSFRs for their $\mu_{*}$ and $f_{\text {gas }}$. Comparisons of the star-forming properties of samples selected either by stellar mass or by $\mathrm{Hi}$ mass must account for the biases imposed on those properties by the various selection criteria.

\section{SUMMARY}

With unprecedented sensitivity, areal coverage, and angular and velocity resolution, the ALFALFA extragalactic $\mathrm{H}$ i survey provides us with a homogeneous parent sample of gas-bearing galaxies that approaches a full census of star-forming galaxthe right-hand side of this equation on the $x$-axis of Figure 15 
The Astronomical Journal, 143:133 (28pp), 2012 June

ies. More than $2 / 3$ of ALFALFA sources are new $\mathrm{Hi}$ detections and thus would not have been included in previous SF studies of large $\mathrm{Hi}$ samples based on optical target selection, e.g., Bothwell et al. (2009). In this paper, we have examined the population of galaxies detected by ALFALFA with the 
lowest $\mathrm{H}$ i masses, combining photometric and spectroscopic data from the SDSS and new UV images from GALEX to probe the relationship between their $\mathrm{H}$ i, stellar, and star-forming components. Compared to other dwarf studies such as FIGGS (Begum et al. 2008) or 11HUGS (Lee et al. 2007), the ALFALFA dwarf sample includes a much larger number of very low $\mathrm{Hi}$ mass objects $\left(\log M_{\mathrm{H}}<7.7\right)$. Moreover, only $56 \%$ of the ALFALFA dwarfs within the SDSS footprint have a counterpart in the SDSS spectroscopic survey, confirming that the ALFALFA survey is providing an important contribution to the census of dwarf galaxies in the nearby universe.

Being sensitive to very low levels of SF activity (below $10^{-2} M_{\cup} \mathrm{yr}^{-1}$ ), the FUV luminosity is a more robust tracer of the SFR in metal- and dust-poor dwarfs than the $\mathrm{H} \alpha$ or IR predictors. We have reprocessed all of the available MIS-level GALEX FUV images for 118 ALFALFA dwarfs. Only one out of those 118 dwarfs is not detected in the FUV: virtually all $\mathrm{H}$ i-selected gasrich dwarfs show some evidence of recent SF. Examinations of the CMD, UV colors, BPT diagram, and $D_{n}(4000)$ all confirm the general notion that $\mathrm{H}$ i-selected dwarf galaxy samples are dominated by faint blue-cloud galaxies and are more likely to be star-forming than galaxies typically included in optical-UVselected samples (e.g., Brinchmann et al. 2004; Salim et al. 2007; Wyder et al. 2007; Catinella et al. 2010).

SED fitting to UV/optical bands is improved via trimming out the models with unrealistically high extinction. We demonstrate that estimates of the $\mathrm{SFR}_{\mathrm{FUV}}$ still suffer from internal extinction in the ALFALFA dwarfs. Even in such lowmetallicity, low-luminosity systems, neglecting the extinction at FUV wavelengths $A_{\mathrm{FUV}}$, as has been the practice of some authors, e.g., Roychowdhury et al. (2009), will lead to the systematic underestimate of FUV luminosity. At the same time, application of the standard IRX- $\beta$ relation given their UV colors would overpredict $A_{\mathrm{FUV}}$ in many of the lowest $\mathrm{Hi}$ mass galaxies, possibly because of their different SFH and/or dust geometry.

Although limited to the SDSS fiber apertures, the SFRs derived from SDSS $\mathrm{H} \alpha$ line flux are compared to those derived from SED fitting, and we cannot rule out systematic deficiency in the SFR values derived from $\mathrm{H} \alpha$ as might be consistent with Lee et al. (2009a). Because the ALFALFA dwarfs have low SFRs on average, they provide an ideal sample for $\mathrm{H} \alpha$ imaging to probe this question; this work is in progress.

Many ALFALFA dwarfs have high SSFRs and $b$-parameters greater than 1, suggesting that they are currently forming stars in a bursty manner. As a result, it is essential to take the possibility of bursty SF into full account when modeling their SEDs. If a constant SFH is assumed, the calibrations used most often will overpredict the derived properties, e.g., the stellar mass as derived from the mass-to-light ratio versus color relation (Bell et al. 2003), or the SFR obtained from the FUV luminosity (Kennicutt 1998a; Lee et al. 2009a).

In the SSFR versus $M_{*}$ diagram, the red sequence galaxies possess low SSFRs. The dispersion in the SSFR distribution increases at both the high- and low-mass ends. Factors other than $M_{*}$ such as environmental effects likely play important roles in the regulation and quenching of SF in low-mass galaxies. In the $f_{\text {gas }}$ versus $M_{*}$ plot, although Bothwell et al. (2009) reported broadening in the $f_{\text {gas }}$ distribution only at the high-mass end, we find a mild increase in the dispersion of $f_{\text {gas }}$ only at the low-mass end. Within the ALFALFA dwarf s-sed sample, Virgo Cluster members have lower gas fractions at a given $M_{\mathrm{H}}$ i with a wide spread also in the distributions of SSFR or the $b$-parameter.
A study of the gas and stars in a larger sample of VCC Virgo dwarfs selected by optical criteria will be presented elsewhere (G. Hallenbeck et al. 2012, in preparation).

A clear decrease of the $f_{\text {gas }}$ with increasing $M_{*}$ is seen for the whole distribution, i.e., on average, the low-mass galaxies are extremely gas-rich for their $M_{*}$ and relatively unevolved. However, because the ALFALFA dwarfs are selected to be the sources with lowest $\mathrm{Hi}$ masses, the imposition of an $\mathrm{H}$ i mass cutoff leads to the selection of a dwarf sample with lower gas fractions for their stellar mass than is characteristic of the $\alpha .40$ sample overall. Therefore, a large portion of the ALFALFA dwarfs studied here still have $T_{\text {cons }} t_{\mathrm{H}}$, in particular the more gas-poor Virgo members.

The H i mass correlates well with the SFR on global scales. Under the simple assumption that the aperture subtended by the half-light radius also contains exactly half of the stellar mass, $\mathrm{H} \mathrm{i}$ mass, and SF, we substitute the general "Kennicutt-Schmidt" SF law (Kennicutt 1998b) into the expression of the SSFR, leading to a predicted relation between SSFR, stellar surface mass density, and gas fraction as given by Equation (5); this relation also depends on the exponent $\alpha$ and scaling factor $C$ in the SF law. The observed trend is consistent with the assumption that $\mathrm{H}$ i disks are in general more extended than the corresponding stellar ones. Additional possible contributions are that ALFALFA galaxies have generally sub-solar metallicity and, probably more importantly, lower SFEs, both of which may lead to lower $C$ values.

A statistical sample such as that provided by ALFALFA allows the study only of global relations between the constituent populations within the galaxies. Clearly, a full understanding of the interaction between gas and stars and the physical mechanisms responsible for laws of SF requires resolved maps of the distribution of $\mathrm{Hi}$ and, where feasible, the molecular component, as well as deeper and better probes of the stellar population. ALFALFA is already providing the fundamental target list for an ongoing Extended Very Large Array/Spitzer/ $H S T$ project exploring the lowest $\mathrm{H}$ i mass galaxies detected by ALFALFA: SHIELD (Survey of $\mathrm{H}$ i in Extremely Low Mass Dwarfs; Cannon et al. 2011). The completion of the ALFALFA survey over the next few years will yield an even richer sample of dwarf galaxies than that studied here.

The authors acknowledge the work of the entire ALFALFA collaboration team in observing, flagging, and extracting the catalog of galaxies used in this work. The ALFALFA team at Cornell is supported by NSF grant AST-0607007 and AST1107390 and by grants from the Brinson Foundation.

GALEX is a NASA Small Explorer, launched in 2003 April. We gratefully acknowledge NASA's support for construction, operation, and science analysis for the GALEX mission, developed in cooperation with the Centre National d'Etudes Spatiales of France and the Korean Ministry of Science and Technology. S.H., S.S., and M.P.H. acknowledge support for this work from the GALEX Guest Investigator program under NASA grants NNX07AJ12G, NNX08AL67G, and NNX09AF79G.

Funding for the SDSS and SDSS-II has been provided by the Alfred P. Sloan Foundation, the Participating Institutions, the National Science Foundation, the US Department of Energy, the NASA, the Japanese Monbukagakusho, the Max Planck Society, and the Higher Education Funding Council for England. The SDSS Web Site is http://www.sdss.org/. The SDSS is managed by the Astrophysical Research Consortium for the Participating Institutions. The Participating Institutions are the 
American Museum of Natural History, Astrophysical Institute Potsdam, University of Basel, University of Cambridge, Case Western Reserve University, University of Chicago, Drexel University, Fermilab, the Institute for Advanced Study, the Japan Participation Group, Johns Hopkins University, the Joint Institute for Nuclear Astrophysics, the Kavli Institute for Particle Astrophysics and Cosmology, the Korean Scientist Group, the Chinese Academy of Sciences (LAMOST), Los Alamos National Laboratory, the Max Planck Institute for Astronomy, the MPA, New Mexico State University, Ohio State University, University of Pittsburgh, University of Portsmouth, Princeton University, the United States Naval Observatory, and the University of Washington.

\section{REFERENCES}

Abazajian, K. N., Adelman-McCarthy, J. K., Agüeros, M. A., et al. 2009, ApJS, 182,543

Asplund, M., Grevesse, N., Sauval, A. J., Allende Prieto, C., \& Kiselman, D. 2004, A\&A, 417, 751

Baldry, I. K., Glazebrook, K., Brinkmann, J., et al. 2004, ApJ, 600, 681

Barnes, D. G., Staveley-Smith, L., de Blok, W. J. G., et al. 2001, MNRAS, 322 , 486

Begum, A., Chengalur, J. N., Karachentsev, I. D., Sharina, M. E., \& Kaisin, S. S. 2008, MNRAS, 386, 1667

Bell, E. F., McIntosh, D. H., Katz, N., \& Weinberg, M. D. 2003, ApJS, 149, 289

Bertin, E., \& Arnouts, S. 1996, A\&AS, 117, 393

Bigiel, F., Leroy, A., Walter, F., et al. 2008, AJ, 136, 2846

Binggeli, B., Sandage, A., \& Tammann, G. A. 1985, AJ, 90, 1681

Blanton, M. R., \& Roweis, S. 2007, AJ, 133, 734

Boissier, S., Boselli, A., Buat, V., Donas, J., \& Milliard, B. 2004, A\&A, 424, 465

Boissier, S., Gil de Paz, A., Boselli, A., et al. 2008, ApJ, 681, 244

Boissier, S., \& Prantzos, N. 2000, MNRAS, 312, 398

Boselli, A., Boissier, S., Cortese, L., \& Gavazzi, G. 2008, ApJ, 674, 742

Bothwell, M. S., Kennicutt, R. C., \& Lee, J. C. 2009, MNRAS, 400, 154

Brinchmann, J., Charlot, S., White, S. D. M., et al. 2004, MNRAS, 351, 1151

Bruzual, G., \& Charlot, S. 2003, MNRAS, 344, 1000

Buat, V., Giovannoli, E., Takeuchi, T. T., et al. 2011, A\&A, 529, A22

Calzetti, D. 1997, AJ, 113, 162

Calzetti, D., Armus, L., Bohlin, R. C., et al. 2000, ApJ, 533, 682

Cannon, J. M., Giovanelli, R., Haynes, M. P., et al. 2011, ApJ, 739, L22

Cannon, J. M., Skillman, E. D., Garnett, D. R., \& Dufour, R. J. 2002, ApJ, 565 , 931

Cardelli, J. A., Clayton, G. C., \& Mathis, J. S. 1989, ApJ, 345, 245

Catinella, B., Schiminovich, D., Kauffmann, G., et al. 2010, MNRAS, 403, 683

Chabrier, G. 2003, PASP, 115, 763

Charlot, S., \& Fall, S. M. 2000, ApJ, 539, 718

Cortese, L., Boselli, A., Buat, V., et al. 2006, ApJ, 637, 242

Draine, B. T., Dale, D. A., Bendo, G., et al. 2007, ApJ, 663, 866

Gallazzi, A., Charlot, S., Brinchmann, J., White, S. D. M., \& Tremonti, C. A. 2005, MNRAS, 362, 41

Galliano, F., Madden, S. C., Jones, A. P., et al. 2003, A\&A, 407, 159

Garnett, D. R. 2002, ApJ, 581, 1019

Gil de Paz, A., Boissier, S., Madore, B. F., et al. 2007, ApJS, 173, 185
Giovanelli, R., Haynes, M. P., Herter, T., et al. 1997, AJ, 113, 22

Giovanelli, R., Haynes, M. P., Kent, B. R., et al. 2005, AJ, 130, 2598

Giovanelli, R., Haynes, M. P., Kent, B. R., et al. 2007, AJ, 133, 2569

Giovanelli, R., Haynes, M. P., Salzer, J. J., et al. 1994, AJ, 107, 2036

Giovanelli, R., Haynes, M. P., Salzer, J. J., et al. 1995, AJ, 110, 1059

Haynes, M. P., Giovanelli, R., Martin, A. M., et al. 2011, AJ, 142, 170

Haynes, M. P., Giovanelli, R., Salzer, J. J., et al. 1999, AJ, 117, 1668

Hopkins, A. M., Miller, C. J., Nichol, R. C., et al. 2003, ApJ, 599, 971

Hunter, D. A., Elmegreen, B. G., \& Ludka, B. C. 2010, AJ, 139, 447

Johnson, B. D., Schiminovich, D., Seibert, M., et al. 2006, ApJ, 644, L109

Johnson, B. D., Schiminovich, D., Seibert, M., et al. 2007, ApJS, 173, 392

Kauffmann, G., Heckman, T. M., White, S. D. M., et al. 2003a, MNRAS, 341, 54

Kauffmann, G., Heckman, T. M., White, S. D. M., et al. 2003b, MNRAS, 341, 33

Kennicutt, R. C., Jr. 1998a, ARA\&A, 36, 189

Kennicutt, R. C., Jr. 1998b, ApJ, 498, 541

Kennicutt, R. C., Jr., Lee, J. C., Funes, S. J., et al. 2008, ApJS, 178, 247

Kim, S., Rey, S.-C., Lisker, T., \& Sohn, S. T. 2010, ApJ, 721, L72

Kong, X., Charlot, S., Brinchmann, J., \& Fall, S. M. 2004, MNRAS, 349, 769

Lee, J. C., Gil de Paz, A., Kennicutt, R. C., Jr., et al. 2011, ApJS, 192, 6

Lee, J. C., Gil de Paz, A., Tremonti, C., et al. 2009a, ApJ, 706, 599

Lee, J. C., Kennicutt, R. C., Jr., Funes, S. J. J. G., Sakai, S., \& Akiyama, S. 2009b, ApJ, 692, 1305

Lee, J. C., Kennicutt, R. C., Funes, S. J., et al. 2007, ApJ, 671, L113

Leroy, A., Bolatto, A., Stanimirovic, S., et al. 2007, ApJ, 658, 1027

Leroy, A., Bolatto, A. D., Simon, J. D., \& Blitz, L. 2005, ApJ, 625, 763

Lisenfeld, U., Hermelo, I., Relano, M., et al. 2011, arXiv:1111.5789

Martin, A. M., Papastergis, E., Giovanelli, R., et al. 2010, ApJ, 723, 1359

Masters, K. L. 2005, PhD thesis, Cornell Univ.

Meurer, G. R., Heckman, T. M., \& Calzetti, D. 1999, ApJ, 521, 64

Morrissey, P., Conrow, T., Barlow, T. A., et al. 2007, ApJS, 173, 682

Popescu, C. C., Tuffs, R. J., Dopita, M. A., et al. 2011, A\&A, 527, A109

Roberts, M. S. 1963, ARA\&A, 1, 149

Roychowdhury, S., Chengalur, J. N., Begum, A., \& Karachentsev, I. D. 2009, MNRAS, 397, 1435

Salim, S., Charlot, S., Rich, R. M., et al. 2005, ApJ, 619, L39

Salim, S., Rich, R. M., Charlot, S., et al. 2007, ApJS, 173, 267

Schiminovich, D., Catinella, B., Kauffmann, G., et al. 2010, MNRAS, 408, 919

Schiminovich, D., Wyder, T. K., Martin, D. C., et al. 2007, ApJS, 173, 315

Schlegel, D. J., Finkbeiner, D. P., \& Davis, M. 1998, ApJ, 500, 525

Shao, Z., Xiao, Q., Shen, S., et al. 2007, ApJ, 659, 1159

Springob, C. M., Haynes, M. P., \& Giovanelli, R. 2005, ApJ, 621, 215

Springob, C. M., Masters, K. L., Haynes, M. P., Giovanelli, R., \& Marinoni, C. 2007, ApJS, 172, 599

Stierwalt, S., Haynes, M. P., Giovanelli, R., et al. 2009, AJ, 138, 338

Thuan, T. X., Sauvage, M., \& Madden, S. 1999, ApJ, 516, 783

Tremonti, C. A., Heckman, T. M., Kauffmann, G., et al. 2004, ApJ, 613, 898

Treyer, M., Schiminovich, D., Johnson, B., et al. 2007, ApJS, 173, 256

Walter, F., Cannon, J. M., Roussel, H., et al. 2007, ApJ, 661, 102

West, A. A., Garcia-Appadoo, D. A., Dalcanton, J. J., et al. 2009, AJ, 138, 796

West, A. A., Garcia-Appadoo, D. A., Dalcanton, J. J., et al. 2010, AJ, 139, 315

Wild, V., Charlot, S., Brinchmann, J., et al. 2011, MNRAS, 417, 1760

Wyder, T. K., Martin, D. C., Schiminovich, D., et al. 2007, ApJS, 173, 293

Xiao, T., Wang, T., Wang, H., et al. 2012, MNRAS, 421, 486

Zhang, W., Li, C., Kauffmann, G., et al. 2009, MNRAS, 397, 1243

Zwaan, M. A., Meyer, M. J., Staveley-Smith, L., \& Webster, R. L. 2005, MNRAS, 359, L30 